\title{
Cul-Catalyzed Suzuki-Miyaura and Sonogashira Cross-Coupling Reactions Using DABCO as Ligand
}

Jin-Heng Li, * Ji-Lan Li, De-Ping Wang, Shao-Feng Pi, Ye-Xiang Xie, Man-Bo Zhang and Xi-Chao $\mathrm{Hu}$

Key Laboratory of Chemical Biology \& Traditional Chinese Medicine Research

(Ministry of Education), College of Chemistry and Chemical Engineering, Hunan

Normal University, Changsha 410081, China

jhli@hunnu.edu.cn

\section{Supporting Information}

\section{List of contents}
(A) Typical Experimental Procedure
S2
(B) Analytical data for 3 and 5
(C) References
S9-10
(D) Spectra
S11-65 


\section{(A) Typical Experimental Procedure}

\section{Typical Experimental Procedure for the CuI/DABCO-catalyzed Suzuki-Type}

\section{Cross-Coupling Reactions}

A mixture of vinyl or aryl halide $\mathbf{1}$ (the indicated amount), boronic acid $\mathbf{2}$ (the indicated amount), $\mathrm{CuI}(10 \mathrm{~mol} \%), \mathrm{DABCO}(20 \mathrm{~mol} \%), \mathrm{Cs}_{2} \mathrm{CO}_{3}$ (2.0 equiv), TBAB (1 equiv) and DMF ( $3 \mathrm{~mL}$ ) in a Schlenk tube was stirred under $\mathrm{N}_{2}$ at $125-130{ }^{\circ} \mathrm{C}$ (oil bar temperature) for desired time (indicated in Table 1, Scheme 2 and Scheme 4) until complete consumption of starting material as monitored by TLC. After the mixture was filtered and evaporated, the residue was purified by flash column chromatography (hexane or hexane/ethyl acetate) to afford the corresponding coupled products.

\section{Typical Experimental Procedure for the CuI/DABCO-catalyzed}

\section{Sonogashira-Type Cross-Coupling Reactions}

A mixture of aryl halide 1 (the indicated amount), alkyne 4 (the indicated amount), $\mathrm{CuI}(10 \mathrm{~mol} \%)$, DABCO (20 mol \%), $\mathrm{Cs}_{2} \mathrm{CO}_{3}$ (2.0 equiv) and DMF (3 mL) in a Schlenk tube was stirred under $\mathrm{N}_{2}$ at $135-140{ }^{\circ} \mathrm{C}$ (oil bar temperature) for desired time (indicated in Table 2, Table 3 and Scheme 3) until complete consumption of starting material as monitored by TLC. After the mixture was filtered and evaporated, the residue was purified by flash column chromatography (hexane or hexane /ethyl acetate) to afford the corresponding coupled products.

\section{(B) Analytical data for 3 and 5}


(E)-1,2-Diphenylethene (3aa): ${ }^{1}{ }^{1} \mathrm{H}$ NMR $\left(300 \mathrm{MHz}, \mathrm{CDCl}_{3}\right) \delta: 7.51(\mathrm{~d}, J=8.4 \mathrm{~Hz}$, 4H), $7.35(\mathrm{t}, J=7.2 \mathrm{~Hz}, 4 \mathrm{H}), 7.27(\mathrm{t}, J=6.3 \mathrm{~Hz}, 2 \mathrm{H}), 7.11(\mathrm{~s}, 2 \mathrm{H}) ;{ }^{13} \mathrm{C}$ NMR $(75$ $\left.\mathrm{MHz}, \mathrm{CDCl}_{3}\right) \delta: 137.3,128.7(2 \mathrm{C}), 127.6,126.5$.

(E)- 1-Chloro-4-styrylbenzene (3ba): ${ }^{1}{ }^{1} \mathrm{H}$ NMR $\left(400 \mathrm{MHz}, \mathrm{CDCl}_{3}\right) \delta: 7.50(\mathrm{~d}, J=$ $7.2 \mathrm{~Hz}, 2 \mathrm{H}), 7.44(\mathrm{~d}, J=8.4 \mathrm{~Hz}, 2 \mathrm{H}), 7.38-7.26(\mathrm{~m}, 5 \mathrm{H}), 7.07(\mathrm{dd}, J=16.4 \mathrm{~Hz}, 16.4$ $\mathrm{Hz}, 2 \mathrm{H}) ;{ }^{13} \mathrm{C} \mathrm{NMR}\left(100 \mathrm{MHz}, \mathrm{CDCl}_{3}\right) \delta: 136.9,135.8,133.1,129.3,128.8,128.7$, $127.9,127.6,127.3,126.5$.

(E)-1-(4-Methoxystyryl)benzene (3ca): ${ }^{1} \mathrm{H}_{\mathrm{NMR}}\left(400 \mathrm{MHz}, \mathrm{CDCl}_{3}\right) \delta: 7.49-7.44$ (m, 4H), $7.34(\mathrm{t}, J=8.0 \mathrm{~Hz}, 2 \mathrm{H}), 7.24-7.23(\mathrm{~m}, 1 \mathrm{H}), 7.02(\mathrm{dd}, J=16.8 \mathrm{~Hz}, 16.4 \mathrm{~Hz}$, 2H), $6.89(\mathrm{~d}, J=8.8 \mathrm{~Hz}, 2 \mathrm{H}), 3.82(\mathrm{~s}, 3 \mathrm{H}) ;{ }^{13} \mathrm{C} \mathrm{NMR}\left(75 \mathrm{MHz}, \mathrm{CDCl}_{3}\right) \delta: 160.0$, $137.7,130.2,128.6,128.2,127.7,127.2,126.6,126.2,114.1,55.3$.

1,1-Diphenylethene (3ea): ${ }^{1} \mathrm{H}$ NMR (400 MHz, $\left.\mathrm{CDCl}_{3}\right) \delta: 7.35-7.30$ (m, 10H), 5.45 (s, 2H); ${ }^{13} \mathrm{C} \mathrm{NMR}\left(100 \mathrm{MHz}, \mathrm{CDCl}_{3}\right) \delta: 150.0,141.5,128.2,128.1,127.7,114.3$.

1-Methoxy-4-(1-phenylvinyl)benzene (3eb): ${ }^{1}{ }^{1} \mathrm{H}$ NMR (400 $\left.\mathrm{MHz}, \mathrm{CDCl}_{3}\right) \quad \delta$ : 7.33-7.31 (m, 5H), $7.27(\mathrm{~d}, J=9.2 \mathrm{~Hz}, 2 \mathrm{H}), 6.86(\mathrm{~d}, J=8.4 \mathrm{~Hz}, 2 \mathrm{H}), 5.39(\mathrm{~s}, 1 \mathrm{H})$, $5.35(\mathrm{~s}, 1 \mathrm{H}), 3.81(\mathrm{~s}, 3 \mathrm{H}) ;{ }^{13} \mathrm{C} \mathrm{NMR}\left(100 \mathrm{MHz}, \mathrm{CDCl}_{3}\right) \delta: 159.3,149.5,141.8,134.0$ $129.4,128.3,128.1,127.6,113.5,112.9,55.3$. 
1-Fluoro-4-(1-phenylvinyl)benzene (3ed): ${ }^{1}{ }^{1} \mathrm{H} \quad \mathrm{NMR} \quad\left(400 \mathrm{MHz}, \mathrm{CDCl}_{3}\right) \quad \delta$ : 7.32-7.27 (m, 7H), $7.00(\mathrm{t}, J=8.1 \mathrm{~Hz}, 2 \mathrm{H}), 5.42(\mathrm{~s}, 1 \mathrm{H}), 5.40(\mathrm{~s}, 1 \mathrm{H}) ;{ }^{13} \mathrm{C} \mathrm{NMR}(75$ $\left.\mathrm{MHz}, \mathrm{CDCl}_{3}\right) \delta: 163.7,161.3,149.0,141.3,137.5,129.9,128.2,128.8,115.0(\mathrm{~d}, J=$ $20.6 \mathrm{~Hz}, 1 \mathrm{C}), 114.2$.

1,3-Piphenylbenzene (3ga): ${ }^{2} \mathrm{H}$ NMR $\left(500 \mathrm{MHz}, \mathrm{CDCl}_{3}\right) \delta: 7.91$ (s, 1H), 7.90-7.73 $(\mathrm{m}, 4 \mathrm{H}), 7.66(\mathrm{t}, J=8.0 \mathrm{~Hz}, 2 \mathrm{H}), 7.60-7.53(\mathrm{~m}, 5 \mathrm{H}), 7.45(\mathrm{~d}, J=8.0 \mathrm{~Hz}, 2 \mathrm{H}) ;{ }^{13} \mathrm{C}$ NMR (125 MHz, $\left.\mathrm{CDCl}_{3}\right) \delta: 141.9,141.3,129.2,128.8,127.4,173.3,126.2(2 \mathrm{C})$.

1,4-Diphenylbenzene (3ha): ${ }^{2}{ }^{1} \mathrm{H}$ NMR $\left(500 \mathrm{MHz}, \mathrm{CDCl}_{3}\right) \delta: 7.72-7.68$ (m, 8H), $7.50(\mathrm{t}, J=7.2 \mathrm{~Hz}, 4 \mathrm{H}), 7.40(\mathrm{t}, J=7.2 \mathrm{~Hz}, 2 \mathrm{H}) ;{ }^{13} \mathrm{C} \mathrm{NMR}\left(125 \mathrm{MHz}, \mathrm{CDCl}_{3}\right) \delta$ : $140.8,140.2,128.8,127.5,127.3,127.0$.

1,3,5-Triphenylbenzene (3ia): ${ }^{2} \mathrm{H}$ NMR $\left(500 \mathrm{MHz}, \mathrm{CDCl}_{3}\right) \delta: 7.84(\mathrm{~s}, 3 \mathrm{H}), 7.75(\mathrm{~d}$, $J=8.0 \mathrm{~Hz}, 6 \mathrm{H}), 7.53(\mathrm{t}, J=7.5 \mathrm{~Hz}, 6 \mathrm{H}), 7.44(\mathrm{t}, J=7.0 \mathrm{~Hz}, 3 \mathrm{H}) ;{ }^{13} \mathrm{C}$ NMR $(125$ $\left.\mathrm{MHz}, \mathrm{CDCl}_{3}\right) \delta: 142.4,141.2,128.8,127.5,127.4,127.3,125.2$.

(E)-1,4-Diphenylbut-1-en-3-yne (5aa): ${ }^{3 \mathrm{j}}{ }^{1} \mathrm{H}$ NMR $\left(400 \mathrm{MHz}, \mathrm{CDCl}_{3}\right) \delta: 7.47$ (d, $J=$ $7.6 \mathrm{~Hz}, 2 \mathrm{H}), 7.41(\mathrm{~d}, J=7.2 \mathrm{~Hz}, 2 \mathrm{H}), 7.33-7.26(\mathrm{~m}, 6 \mathrm{H}), 7.03(\mathrm{~d}, J=16.4 \mathrm{~Hz}, 1 \mathrm{H})$, $6.37(\mathrm{~d}, J=16.0 \mathrm{~Hz}, 1 \mathrm{H}) ;{ }^{13} \mathrm{C} \mathrm{NMR}\left(100 \mathrm{MHz}, \mathrm{CDCl}_{3}\right) \delta: 141.2,136.3,131.5,128.7$, $128.6,128.3,128.2,126.3,123.4,108.1,91.8,88.9$. 
(E)-1-Chloro-4-(4-phenylbut-1-en-3-ynyl)benzene (5ba): ${ }^{\mathbf{1}, 3}{ }^{1} \mathrm{H}$ NMR (400 MHz, $\left.\mathrm{CDCl}_{3}\right) \delta: 7.48-7.45(\mathrm{~m}, 2 \mathrm{H}), 7.33-7.30(\mathrm{~m}, 7 \mathrm{H}), 6.97(\mathrm{~d}, J=16.0 \mathrm{~Hz}, 1 \mathrm{H}), 6.34(\mathrm{~d}, J$ $=16.0 \mathrm{~Hz}, 1 \mathrm{H}) ;{ }^{13} \mathrm{C} \mathrm{NMR}\left(100 \mathrm{MHz}, \mathrm{CDCl}_{3}\right) \delta: 139.8,134.8,134.2,131.5,128.9$, 128.4, 128.3 (2C), 127.4, 108.8, 92.3, 88.6.

(E)-1-(Non-1-en-3-ynyl)benzene (5da): ${ }^{1}{ }^{1} \mathrm{H}$ NMR (400 MHz, $\left.\mathrm{CDCl}_{3}\right) \delta: 7.35(\mathrm{t}, J=$ $8.4 \mathrm{~Hz}, 2 \mathrm{H}), 7.31(\mathrm{t}, J=8.0 \mathrm{~Hz}, 2 \mathrm{H}), 7.26(\mathrm{~d}, J=7.2 \mathrm{~Hz}, 1 \mathrm{H}), 6.87(\mathrm{~d}, J=16.0 \mathrm{~Hz}$, $1 \mathrm{H}), 6.16(\mathrm{~d}, J=16.0 \mathrm{~Hz}, 1 \mathrm{H}), 2.36(\mathrm{t}, J=6.8 \mathrm{~Hz}, 2 \mathrm{H}), 1.59-1.56(\mathrm{~m}, 2 \mathrm{H}), 1.41-1.34$ (m, 4H), $0.92(\mathrm{t}, J=7.2 \mathrm{~Hz}, 3 \mathrm{H}) ;{ }^{13} \mathrm{C} \mathrm{NMR}\left(100 \mathrm{MHz}, \mathrm{CDCl}_{3}\right) \delta: 139.9,136.5,128.5$, $128.1,125.9,108.8,93.0,80.0,31.0,28.4,22.2,19.5,13.9$.

1-Methoxy-4-(2-phenylethynyl)benzene (5ja): ${ }^{3} \mathrm{H}$ NMR $\left(400 \mathrm{MHz}, \mathrm{CDCl}_{3}\right) \delta$ : 7.52-7.51 (m, 2H), $7.48(\mathrm{~d}, J=9.0 \mathrm{~Hz}, 2 \mathrm{H}), 7.34-7.32(\mathrm{~m}, 3 \mathrm{H}), 6.88(\mathrm{~d}, J=8.8 \mathrm{~Hz}$, 2H), $3.83(\mathrm{~s}, 3 \mathrm{H}) ;{ }^{13} \mathrm{C}$ NMR $\left(100 \mathrm{MHz}, \mathrm{CDCl}_{3}\right) \delta: 159.6,133.0,131.4,128.3,127.9$, 123.6, 115.4, 114.0, 89.4, 88.1, 55.3.

1-(Dec-1-ynyl)-4-methoxybenzene (5jb): ${ }^{3} \mathrm{H}$ NMR $\left(400 \mathrm{MHz}, \mathrm{CDCl}_{3}\right) \delta: 7.33$ (d, $J$ $=8.8 \mathrm{~Hz}, 2 \mathrm{H}), 6.81(\mathrm{~d}, J=8.8 \mathrm{~Hz}, 2 \mathrm{H}), 3.80(\mathrm{~s}, 3 \mathrm{H}), 2.38(\mathrm{t}, J=7.6 \mathrm{~Hz}, 2 \mathrm{H})$, $1.61-1.57(\mathrm{~m}, 2 \mathrm{H}), 1.45-1.40(\mathrm{~m}, 2 \mathrm{H}), 1.31-1.24(\mathrm{~m}, 8 \mathrm{H}), 0.88(\mathrm{t}, J=6.4 \mathrm{~Hz}, 3 \mathrm{H})$; 
${ }^{13} \mathrm{C}$ NMR (100 MHz, $\left.\mathrm{CDCl}_{3}\right) \delta: 158.9,132.8,116.1,113.7,88.8,80.1,55.2,31.8,29.2$, $29.1,28.9,28.8,22.7,19.4,14.1$.

2-(3-(4-Methoxyphenyl)prop-2-ynyloxy)-tetrahydro-2H-pyran (5jc): ${ }^{3}{ }^{1} \mathrm{H} \quad \mathrm{NMR}$ $\left(400 \mathrm{MHz}, \mathrm{CDCl}_{3}\right) \delta: 7.39(\mathrm{~d}, J=8.8 \mathrm{~Hz}, 2 \mathrm{H}), 6.83(\mathrm{~d}, J=8.8 \mathrm{~Hz}, 2 \mathrm{H}), 4.90(\mathrm{t}, J=$ $3.2 \mathrm{~Hz}, 1 \mathrm{H}), 4.53-4.22(\mathrm{~m}, 2 \mathrm{H}), 3.89(\mathrm{t}, J=7.2 \mathrm{~Hz}, 1 \mathrm{H}), 3.80(\mathrm{~s}, 3 \mathrm{H}), 3.58-3.54(\mathrm{~m}$, 1H), 1.76-1.54 (m, 6H); ${ }^{13} \mathrm{C}$ NMR (100 MHz, $\left.\mathrm{CDCl}_{3}\right) \delta: 159.6,133.3,114.8,113.8$, $96.8,85.7,83.6,62.0,55.2,54.8,30.3,25.4,19.1$.

1-(2-(4-Nitrophenyl)ethynyl)benzene (5ka): ${ }^{3} \mathrm{H}$ NMR $\left(300 \mathrm{MHz}, \mathrm{CDCl}_{3}\right) \delta: 8.21$ (d, $J=8.8 \mathrm{~Hz}, 2 \mathrm{H}), 7.65(\mathrm{~d}, J=8.8 \mathrm{~Hz}, 2 \mathrm{H}), 7.58-7.54(\mathrm{~m}, 2 \mathrm{H}), 7.40-7.38(\mathrm{~m}, 3 \mathrm{H}),{ }^{13} \mathrm{C}$ NMR (75 MHz, $\left.\mathrm{CDCl}_{3}\right)$ \&: 141.3, 132.6, 132.2, 130.6, 129.6, 128.8, 124.0, 122.5, 95.0, 87.9. Elem. Anal.: calcd. C, 75.33; H, 4.06; N, 6.27, found C, 75.29; H, 4.09; N, 6.25

1-(Dec-1-ynyl)-4-nitrobenzene (5kb): ${ }^{3}{ }^{1} \mathrm{H}$ NMR $\left(400 \mathrm{MHz}, \mathrm{CDCl}_{3}\right) \delta: 8.15(\mathrm{~d}, J=$ $8.8 \mathrm{~Hz}, 2 \mathrm{H}), 7.51(\mathrm{~d}, J=8.8 \mathrm{~Hz}, 2 \mathrm{H}), 2.44(\mathrm{t}, J=7.2 \mathrm{~Hz}, 2 \mathrm{H}), 1.74-1.59(\mathrm{~m}, 2 \mathrm{H})$, $1.47-1.43(\mathrm{~m}, 2 \mathrm{H}), 1.31-1.26(\mathrm{~m}, 8 \mathrm{H}), 0.89(\mathrm{t}, J=7.2 \mathrm{~Hz}, 3 \mathrm{H}) ;{ }^{13} \mathrm{C} \mathrm{NMR}(100 \mathrm{MHz}$, $\left.\mathrm{CDCl}_{3}\right) \delta: 132.6,131.6,123.8,118.5,97.2,79.6,32.2,29.5,29.4,29.3,28.7,23.0$, $19.9,14.4$. 
1-(2-(2-Nitrophenyl)ethynyl)benzene (5la): ${ }^{3}{ }^{1} \mathrm{H} \mathrm{NMR}\left(300 \mathrm{MHz}, \mathrm{CDCl}_{3}\right) \delta: 8.10(\mathrm{~d}$, $J=8.4 \mathrm{~Hz}, 1 \mathrm{H}), 7.73(\mathrm{~d}, J=7.6 \mathrm{~Hz}, 1 \mathrm{H}), 7.64-7.60(\mathrm{~m}, 3 \mathrm{H}), 7.48(\mathrm{t}, J=8.4 \mathrm{~Hz}, 1 \mathrm{H})$, 7.40-7.38 (m, 3H); ${ }^{13} \mathrm{C}$ NMR (75 MHz, $\left.\mathrm{CDCl}_{3}\right) \delta: 149.5,132.9,132.0,129.2,128.5$, $128.4,124.7,122.3,118.7,97.1,84.8$.

1,2-Diphenylethyne (5ma): ${ }^{3} \mathrm{H}$ NMR (300 MHz, $\left.\mathrm{CDCl}_{3}\right) \delta: 7.60-7.51(\mathrm{~m}, 4 \mathrm{H})$, 7.39-7.26 (m, 6H); ${ }^{13} \mathrm{C} \mathrm{NMR}\left(75 \mathrm{MHz}, \mathrm{CDCl}_{3}\right) \delta: 132.0,128.7,128.6,123.7,89.7$.

1-(2-p-Tolylethynyl)benzene (5na): ${ }^{3}{ }^{1} \mathrm{H}$ NMR $\left(300 \mathrm{MHz}, \mathrm{CDCl}_{3}\right) \delta: 7.54-7.50(\mathrm{~m}$, 2H), $7.43(\mathrm{~d}, J=8.1 \mathrm{~Hz}, 2 \mathrm{H}), 7.35-7.31(\mathrm{~m}, 3 \mathrm{H}), 7.15(\mathrm{~d}, J=7.8 \mathrm{~Hz}, 2 \mathrm{H}), 2.36(\mathrm{~s}$, $3 \mathrm{H}) ;{ }^{13} \mathrm{C} \mathrm{NMR}\left(100 \mathrm{MHz}, \mathrm{CDCl}_{3}\right) \delta: 138.7,132.9,131.9,129.6,129.5,128.8,128.7$, $128.4,89.1,83.4,21.9$.

1-(Dec-1-ynyl)-4-methylbenzene (5nb): ${ }^{3} \mathrm{H}$ NMR $\left(400 \mathrm{MHz}, \mathrm{CDCl}_{3}\right) \delta: 7.28(\mathrm{~d}, J=$ $8.0 \mathrm{~Hz}, 2 \mathrm{H}), 7.08(\mathrm{~d}, J=8.0 \mathrm{~Hz}, 2 \mathrm{H}), 2.38(\mathrm{t}, J=7.2 \mathrm{~Hz}, 2 \mathrm{H}), 2.32(\mathrm{~s}, 3 \mathrm{H}), 1.61-1.55$ $(\mathrm{m}, 2 \mathrm{H}), 1.46-1.42(\mathrm{~m}, 2 \mathrm{H}), 1.31-1.29(\mathrm{~m}, 8 \mathrm{H}), 0.89(\mathrm{t}, J=7.2 \mathrm{~Hz}, 3 \mathrm{H}) ;{ }^{13} \mathrm{C} \mathrm{NMR}$ $\left(100 \mathrm{MHz}, \mathrm{CDCl}_{3}\right) \delta: 137.4,131.4,128.9,121.0,89.6,80.6,31.9,29.2,29.1,28.9$, $28.8,23.7,21.4,18.4,14.1$

1-(2-o-Tolylethynyl)benzene (5oa): ${ }^{2} \mathrm{H}$ NMR (400 MHz, $\left.\mathrm{CDCl}_{3}\right) \delta: 7.53-7.52(\mathrm{~m}$, 3H), 7.35-7.33 (m, 4H), 7.23-7.22 (m, 2H) $2.52(\mathrm{~s}, 3 \mathrm{H}) ;{ }^{13} \mathrm{C} \mathrm{NMR}\left(100 \mathrm{MHz}, \mathrm{CDCl}_{3}\right)$ 
$\delta: 140.2,132.6,131.9,131.6,129.5,129.2,128.4,128.3,128.2,125.6,88.4,81.7$, 20.7 .

1-(2-(4-Methoxyphenyl)ethynyl)-4-nitrobenzene (5pd): ${ }^{3}{ }^{1} \mathrm{H}$ NMR (400 MHz, $\left.\mathrm{CDCl}_{3}\right) \delta: 8.20(\mathrm{~d}, J=8.4 \mathrm{~Hz}, 2 \mathrm{H}), 7.63(\mathrm{~d}, J=8.8 \mathrm{~Hz}, 2 \mathrm{H}), 7.50(\mathrm{~d}, J=8.4 \mathrm{~Hz}, 2 \mathrm{H})$, $6.90(\mathrm{~d}, J=8.4 \mathrm{~Hz}, 2 \mathrm{H}), 3.84(\mathrm{~s}, 3 \mathrm{H}) ;{ }^{13} \mathrm{C} \mathrm{NMR}\left(100 \mathrm{MHz}, \mathrm{CDCl}_{3}\right) \delta: 160.4,146.7$, 134.0, 133.4, 132.0, 123.6, 114.2, 114.1, 95.1, 86.6, 55.3.

1-Fluoro-4-(2-(4-nitrophenyl)ethynyl)benzene (5pe): ${ }^{3} \mathrm{H}$ NMR (400 $\left.\mathrm{MHz}, \mathrm{CDCl}_{3}\right)$ $\delta: 8.23(\mathrm{~d}, J=8.0 \mathrm{~Hz}, 2 \mathrm{H}), 7.66(\mathrm{~d}, J=8.4 \mathrm{~Hz}, 2 \mathrm{H}), 7.57-7.54(\mathrm{~m}, 2 \mathrm{H}), 7.10(\mathrm{t}, J=$ 8.8 Hz, 2H); $\left.{ }^{13} \mathrm{C} \mathrm{NMR} \mathrm{(100} \mathrm{MHz,} \mathrm{CDCl}_{3}\right) \delta: 164.3,161.8,146.9,133.8,132.2,130.0$, $123.7,116.0(\mathrm{~d}, \mathrm{~J}=22 \mathrm{~Hz}, 1 \mathrm{C}), 93.6,87.3$.

2-Phenylbenzofuran (5va): ${ }^{3} \mathrm{H}$ NMR $(400 \mathrm{MHz}) \delta: 7.87(\mathrm{~d}, J=7.2 \mathrm{~Hz}, 2 \mathrm{H}), 7.58$ (d, $J=7.6 \mathrm{~Hz}, 1 \mathrm{H}), 7.52(\mathrm{~d}, J=8.0 \mathrm{~Hz}, 1 \mathrm{H}), 7.45(\mathrm{t}, J=7.2 \mathrm{~Hz}, 2 \mathrm{H}), 7.33(\mathrm{t}, J=7.2 \mathrm{~Hz}$, 1H), $7.28(\mathrm{t}, J=7.2 \mathrm{~Hz}, 1 \mathrm{H}), 7.23(\mathrm{t}, J=7.2 \mathrm{~Hz}, 1 \mathrm{H}), 7.03(\mathrm{~s}, 1 \mathrm{H}) ;{ }^{13} \mathrm{C} \mathrm{NMR}(100$ MHz) $\delta: 155.9,154.9,130.4,129.2,128.8,128.5,124.9,124.2,122.9,120.9,111.2$, 101.3.

2-Octylbenzofuran (5vb): ${ }^{1} \mathrm{H}$ NMR $\left(400 \mathrm{MHz}, \mathrm{CDCl}_{3}\right) \delta: 7.48(\mathrm{~d}, J=8.0 \mathrm{~Hz}, 1 \mathrm{H})$, $7.41(\mathrm{~d}, J=8.4 \mathrm{~Hz}, 1 \mathrm{H}), 7.22-7.15(\mathrm{~m}, 2 \mathrm{H}), 6.37(\mathrm{~s}, 1 \mathrm{H}), 2.76(\mathrm{t}, J=7.6 \mathrm{~Hz}, 2 \mathrm{H})$, $1.77-1.70(\mathrm{~m}, 2 \mathrm{H}), 1.41-1.27(\mathrm{~m}, 10 \mathrm{H}), 0.88(\mathrm{t}, J=6.8 \mathrm{~Hz}, 3 \mathrm{H}) ;{ }^{13} \mathrm{C}$ NMR $(100$ 
$\left.\mathrm{MHz}, \mathrm{CDCl}_{3}\right) \delta: 159.8,154.6,129.0,123.0,122.3,120.1,110.7,101.7,31.8,29.3$, 29.2, 28.4, 27.7, 22.7, 14.1; LRMS (EI, $70 \mathrm{eV}) \mathrm{m} / z(\%): 230\left(\mathrm{M}^{+}, 64\right), 187$ (11), 173 (8), 145 (13), 131 (100); HRMS (EI) for $\mathrm{C}_{16} \mathrm{H}_{22} \mathrm{O}\left(\mathrm{M}^{+}\right)$: calcd. 230.1680, found 230.1678 .

2-Phenylethynylaniline (5wa): ${ }^{4}{ }^{1} \mathrm{H}$ NMR $\left(400 \mathrm{MHz}, \mathrm{CDCl}_{3}\right) \delta: 7.53(\mathrm{~d}, J=9.6 \mathrm{~Hz}$, 2H), 7.38-7.33 (m, 4H), $7.14(\mathrm{t}, J=7.6 \mathrm{~Hz}, 1 \mathrm{H}), 6.72(\mathrm{~d}, J=8.0 \mathrm{~Hz}, 2 \mathrm{H}), 4.28$ (brs, $2 \mathrm{H}) ;{ }^{13} \mathrm{C} \mathrm{NMR}\left(100 \mathrm{MHz}, \mathrm{CDCl}_{3}\right) \delta: 147.6,132.1,131.5,129.7,128.4,128.2,123.3$, 118.1, 114.4, 108. 0, 94.7, 85.8.

\section{(C) References}

(1) Zeni, G.; Braga, A. L.; Stefani, H. A.; Acc. Chem. Res. 2003, 36, 731 and references cited therein.

(2) Liu, L.; Zhang, Y.; Xin, B. J. Org. Chem. 2006, 71, 3394.

(3) (a) Okuro, K.; Furuune, M.; Enna, M.; Miura, M.; Nomura, M. J. Org. Chem. 1993, 58, 4716. (b) Gujadhur, R. K.; Bates, C. G.; Venkataraman, D. Org. Lett. 2001, 3, 4315. (c) Ma, D, Liu, F. Chem. Commun. 2004, 1934. (d) Saejueng, P.; Bates, C. G.; Venkataraman, D. Synthesis. 2005, 1706. (1) Böhm, V. P. W.; Herrmann, W. A. Eur. J. Org. Chem. 2000, 3679. (e) Netherton, M. R.; Fu, G. C. Org. Lett. 2001, 3, 4295. (f) Méry, D.; Heuzé, K.; Astrc, D. Chem. Commun. 2003, 1934. (g) Gelman, D.; Buthwald, S. L. Angew. Chem. Int. Ed. 2003, 42, 5993. (h) Wolf, C.; Lerebours, R. Org. Biomol. Chem. 2004, 2, 2161. (i) Heuzé, K.; Méry, 
D.; Gauss, D.; Blais, J.-C.; Astruc, D. Chem. Eur. J. 2004, 10, 3936. (j) Feuerstein, M.; Berthiol, F.; Doucet, H.; Santelli, M. Synthesis 2004, 1281.

(4) Arcadi, A.; Marinelli, F. Synthesis 1986, 749. 


\section{(D) Spectra}

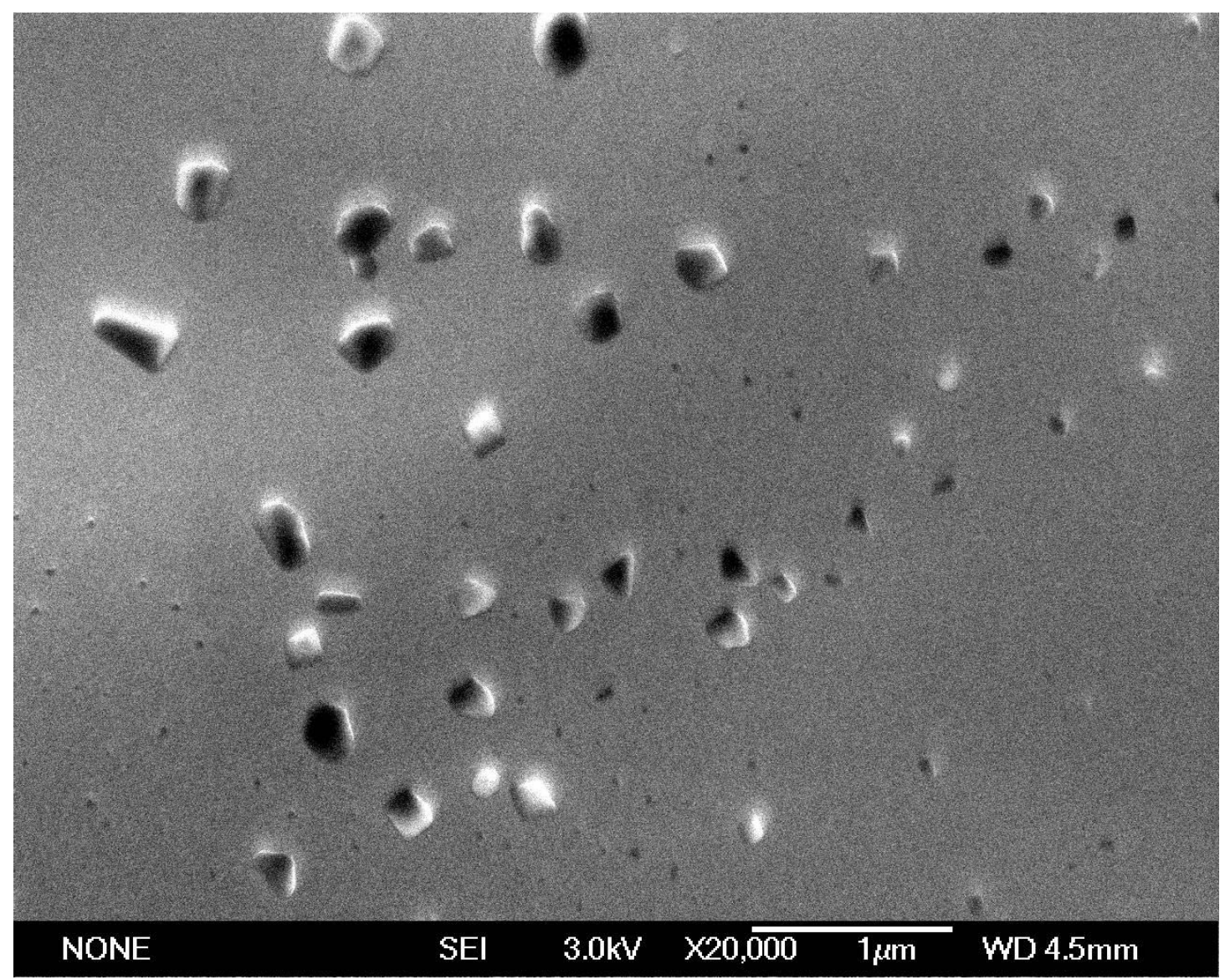

Figure 1. TEM picture of $\mathrm{CuI}(\mathrm{CuI}$ was obtained under thermal conditions in the presence of $n$ - $\mathrm{Bu}_{4} \mathrm{NBr}$, and it was not nano-scale based on the picture) 
(E)-1,2-diphenylethene (3aa)

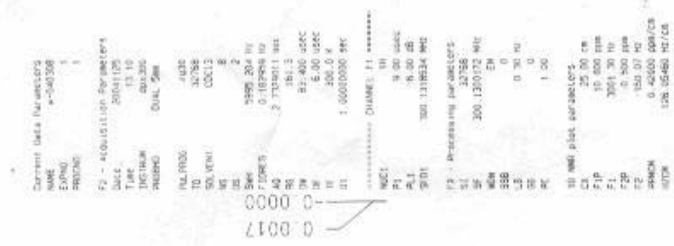

01 t 1

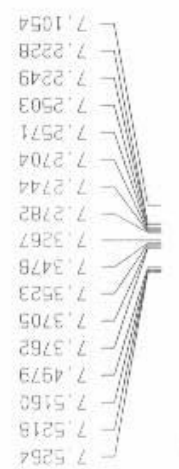

$\log$

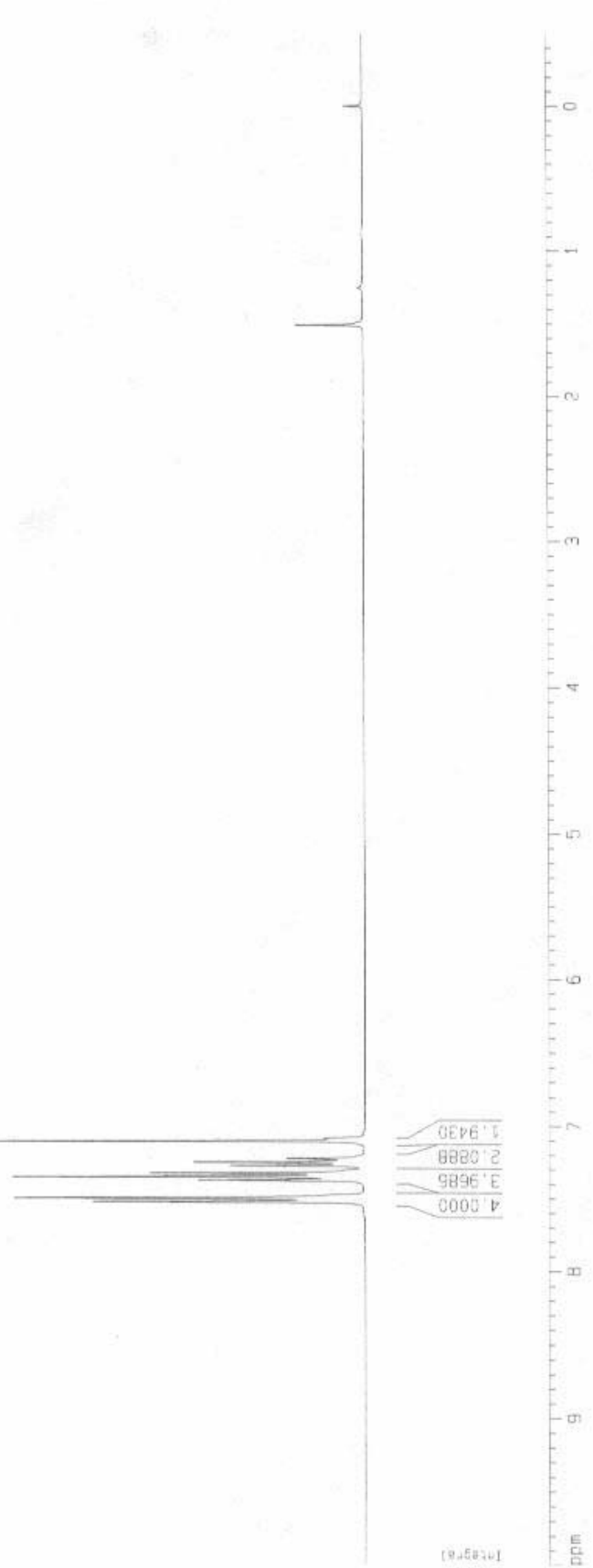


(E)-1,2-diphenylethene (3aa)

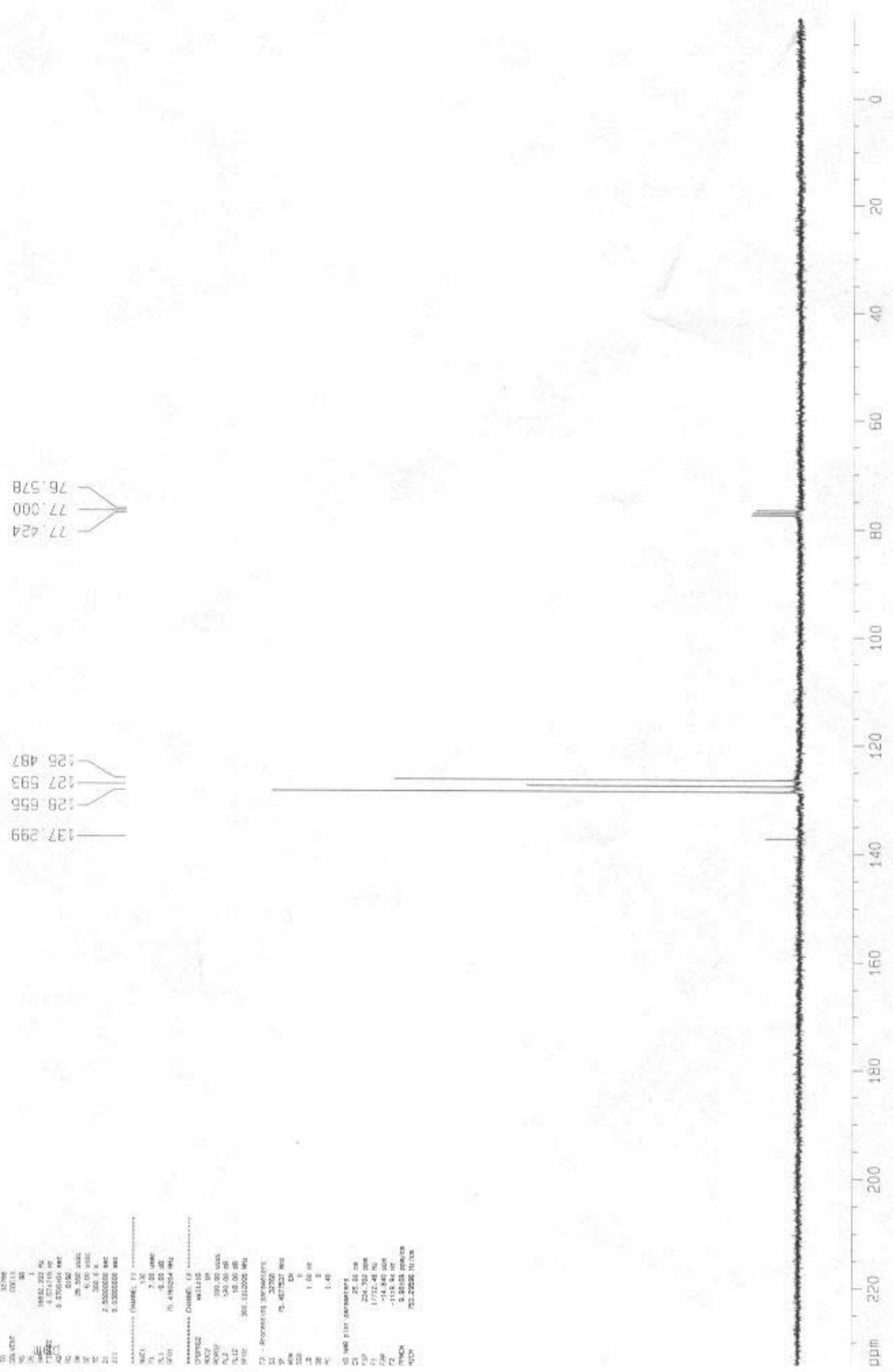


(E)- 1-chloro-4-styrylbenzene (3ba)

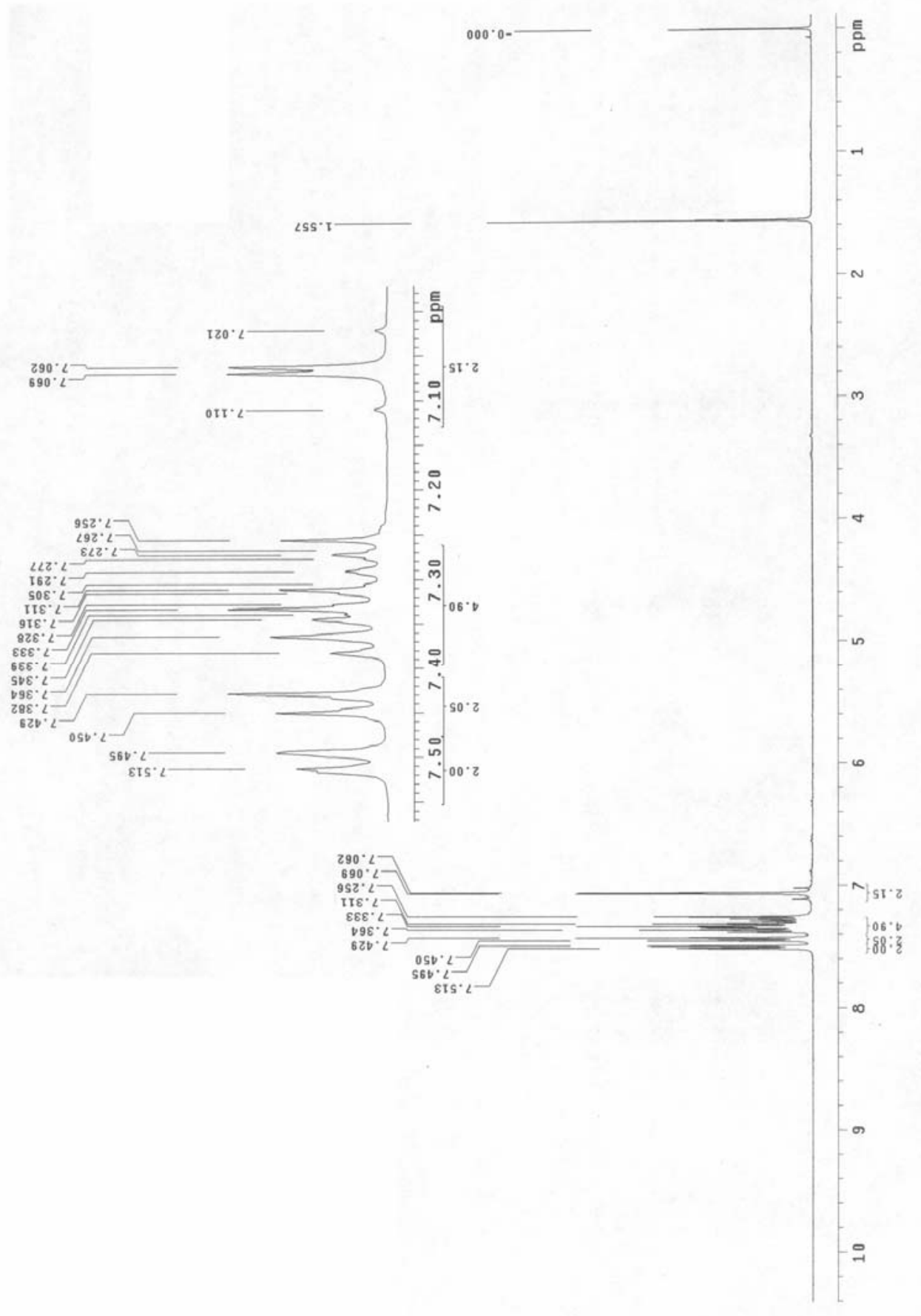


(E)- 1-chloro-4-styrylbenzene (3ba)

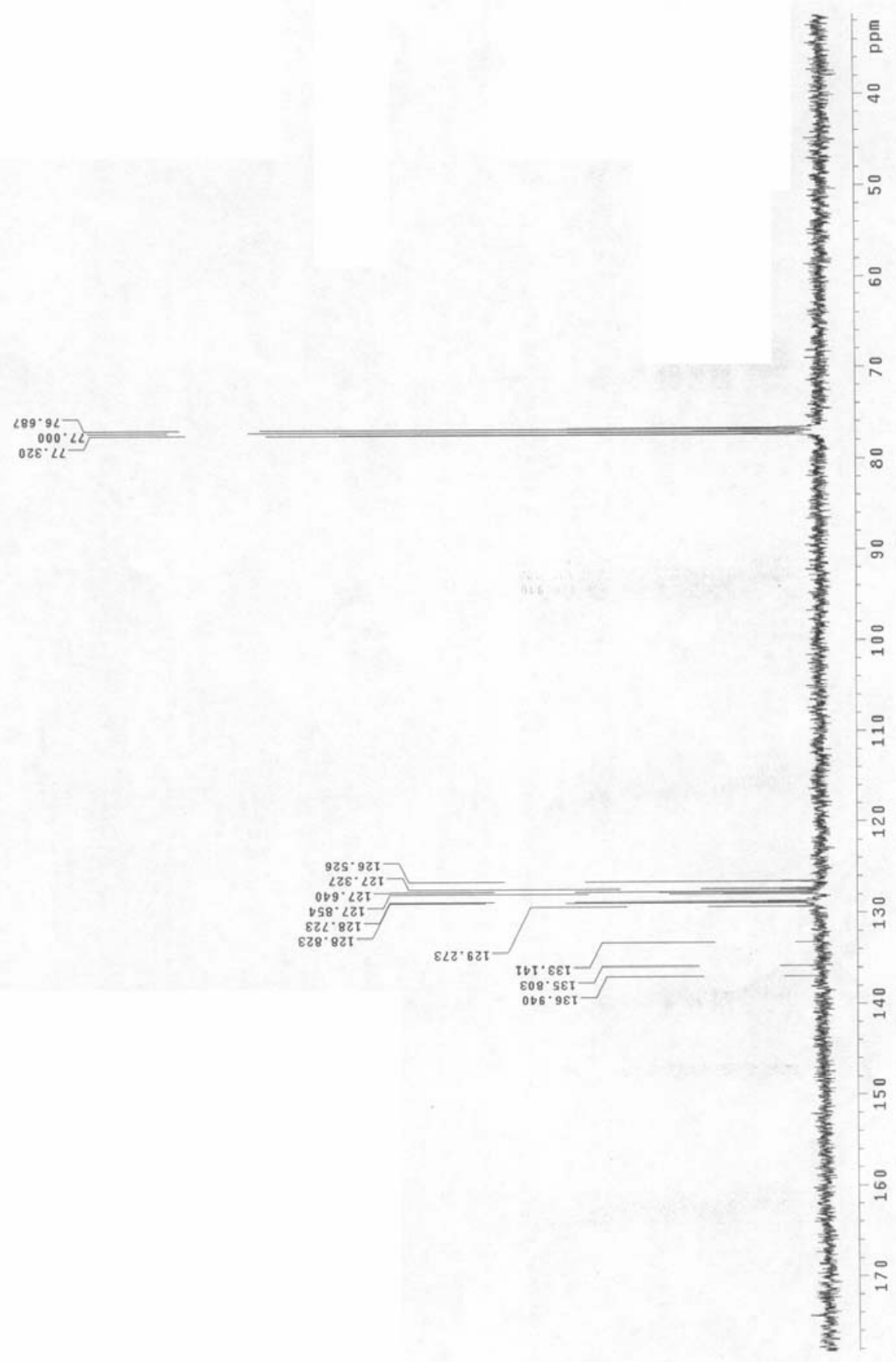


(E)-1-(4-methoxystyryl)benzene (3ca)

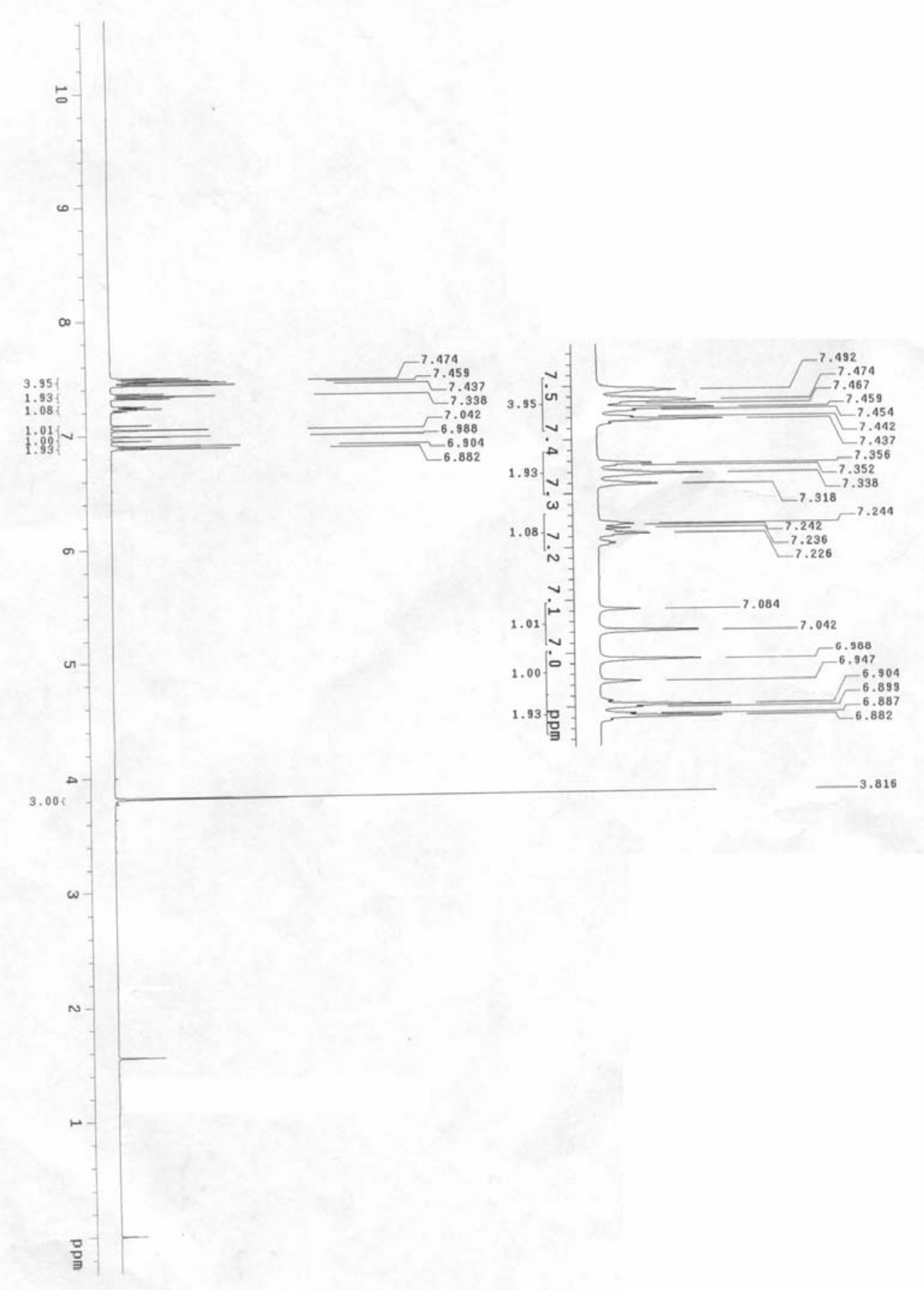


(E)-1-(4-methoxystyryl)benzene (3ca)

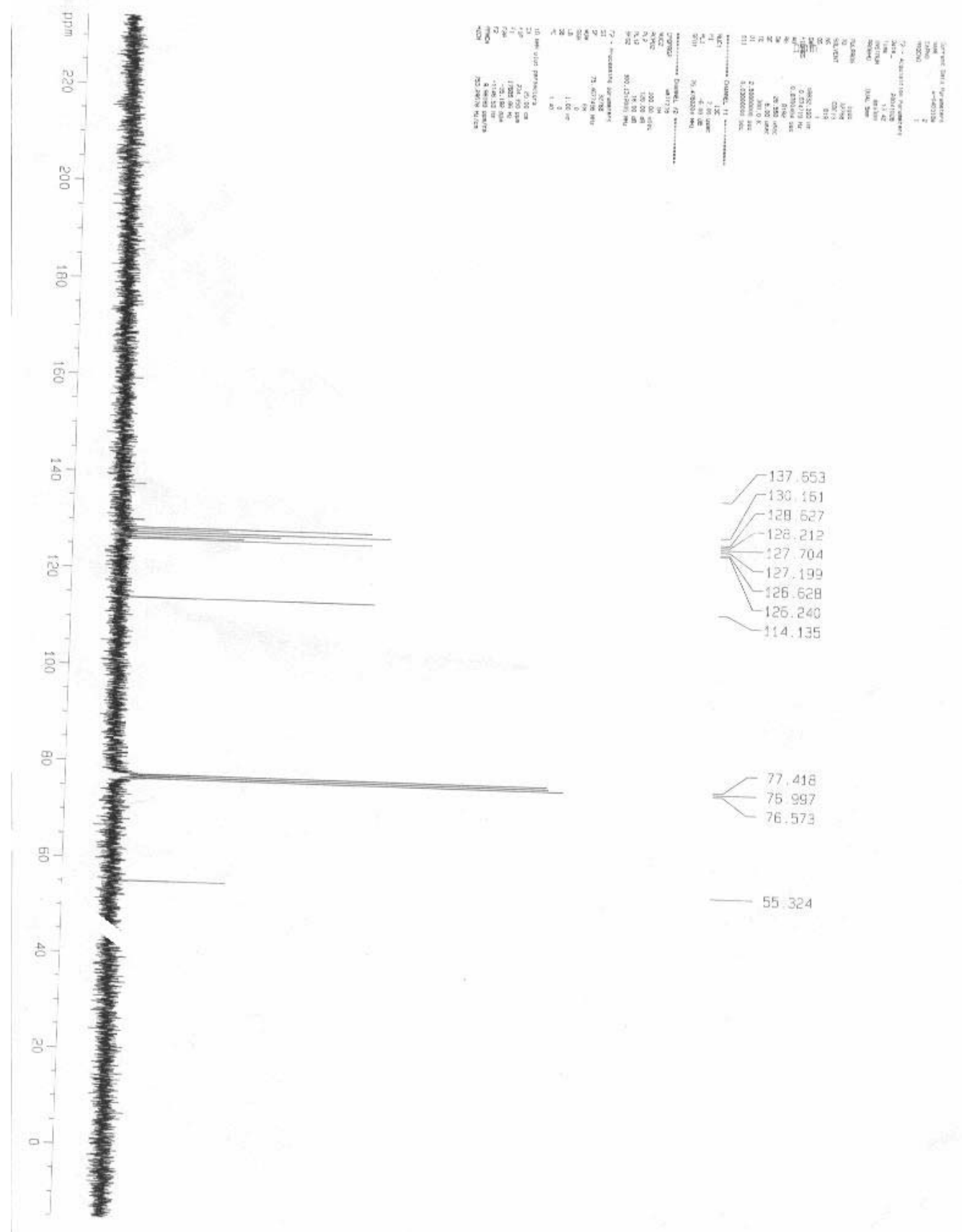


1,1-diphenylethene (3ea)

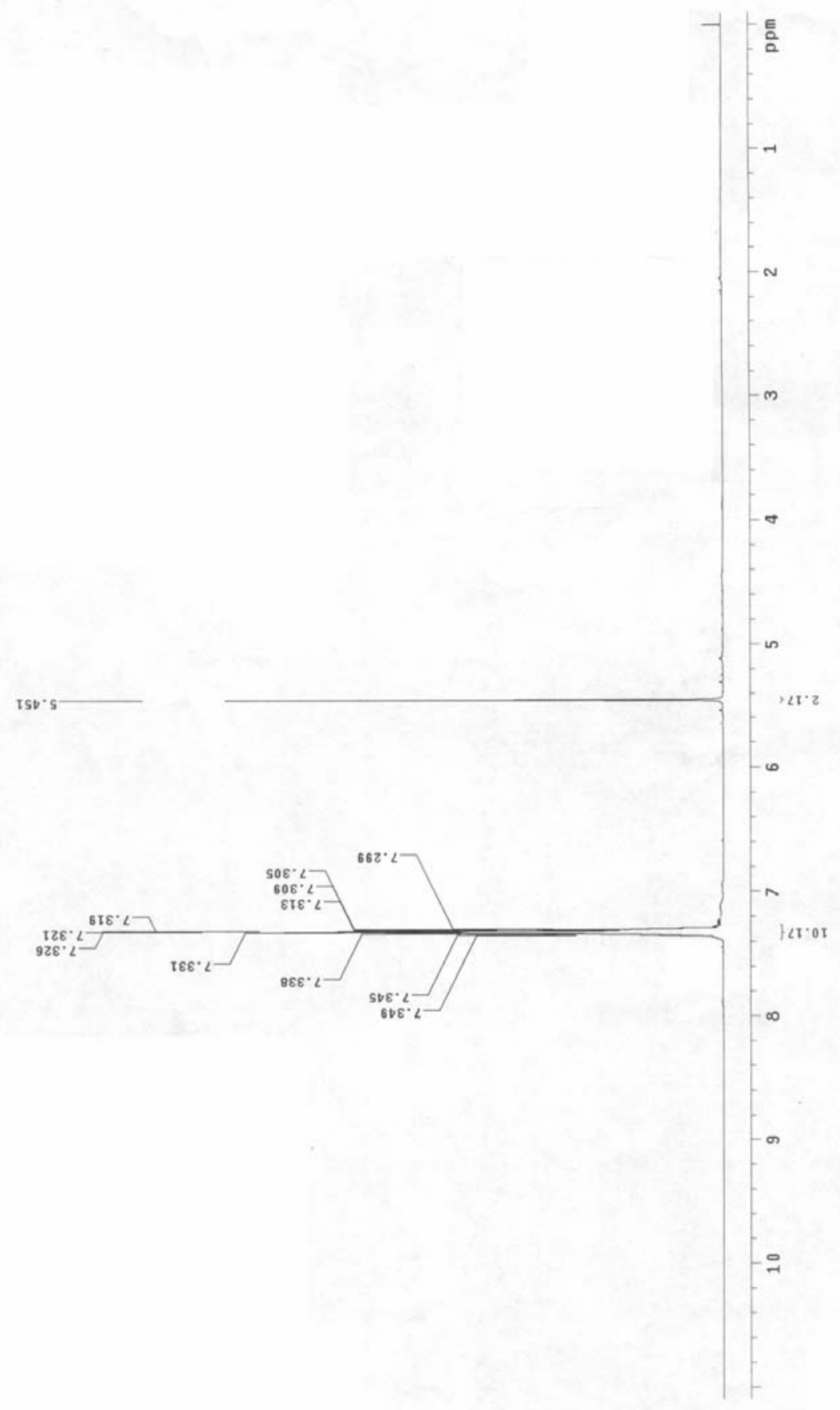


1,1-diphenylethene (3ea)

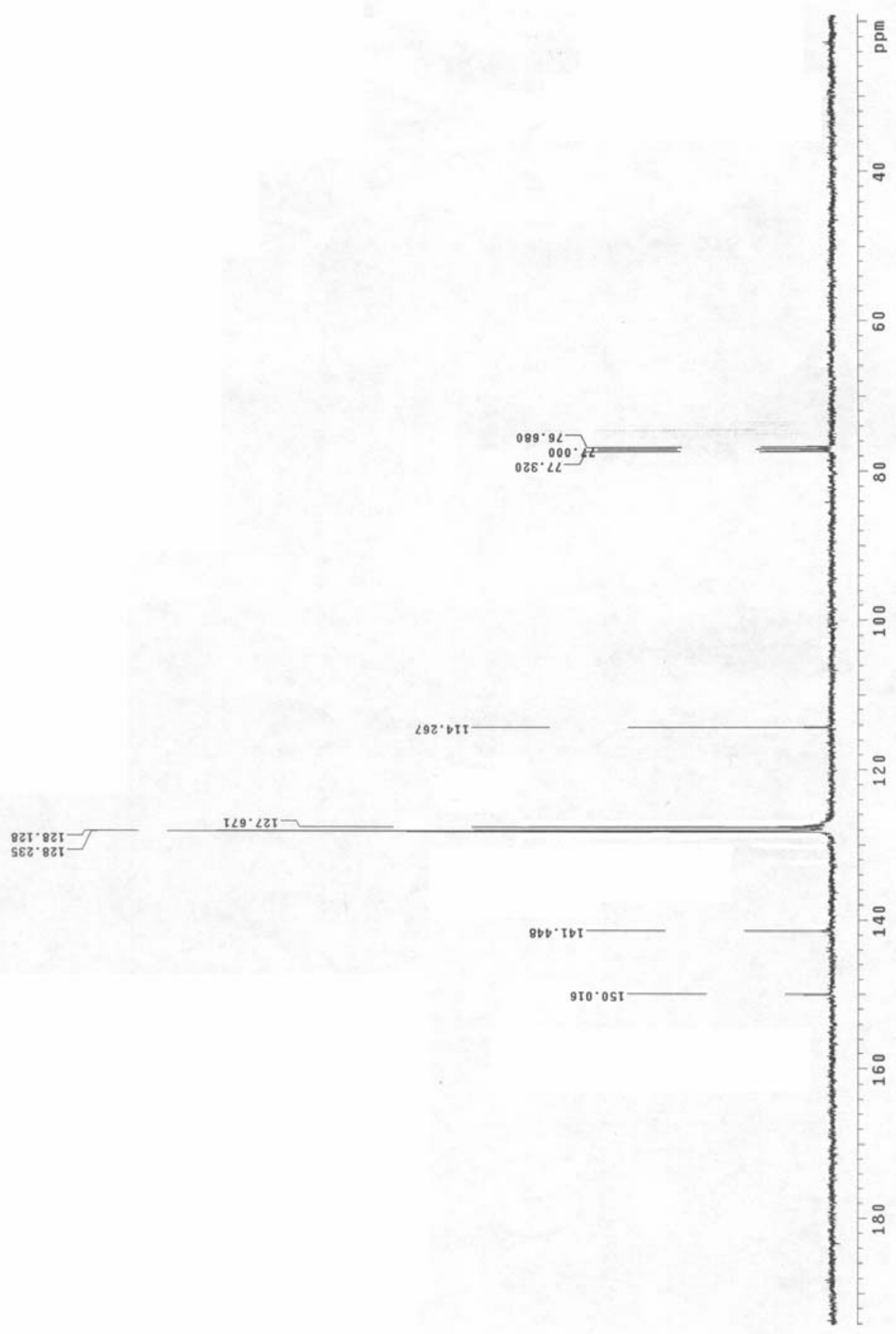


1-Methoxy-4-(1-phenylvinyl)benzene (3eb)

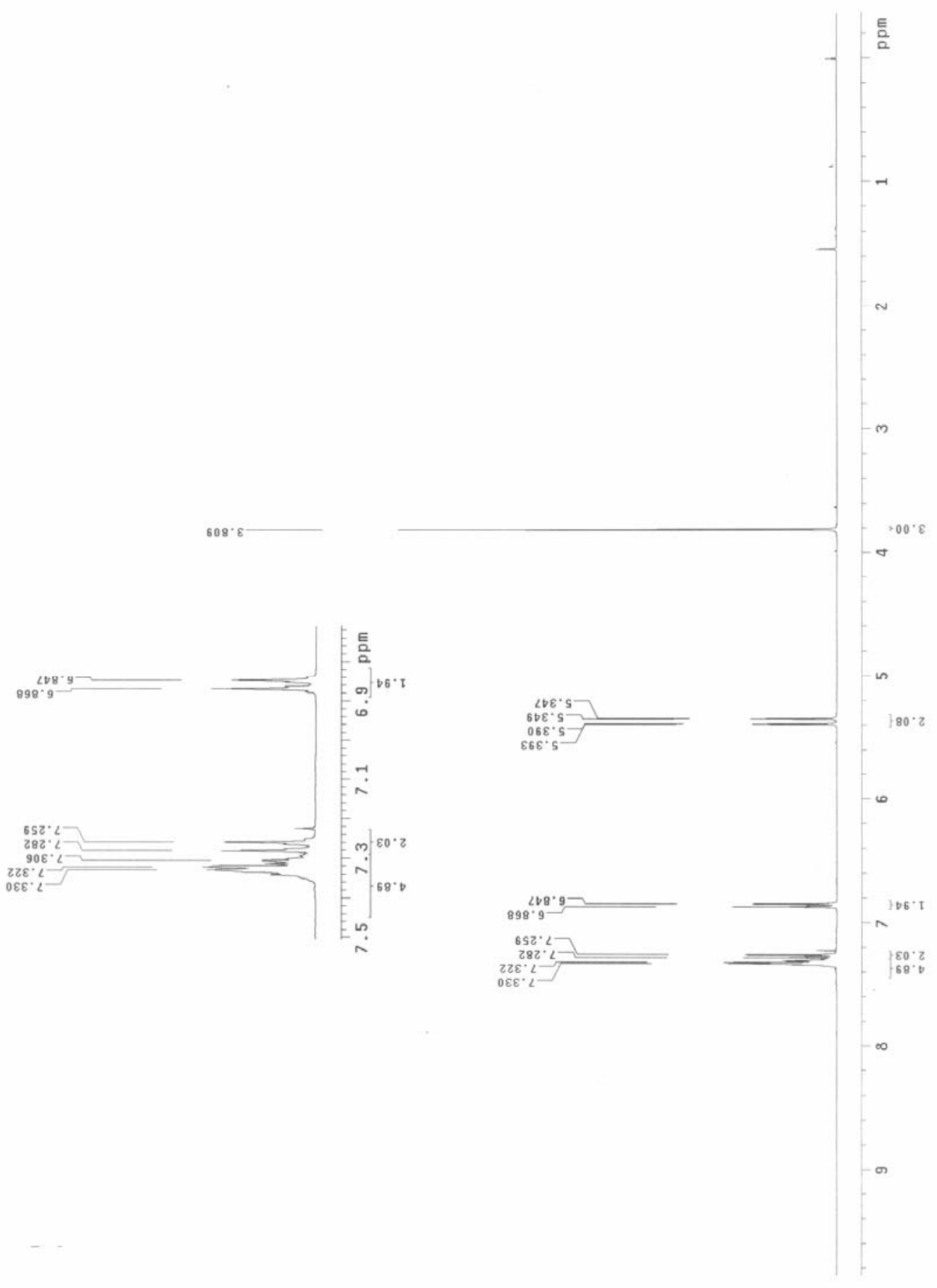


1-Methoxy-4-(1-phenylvinyl)benzene (3eb)

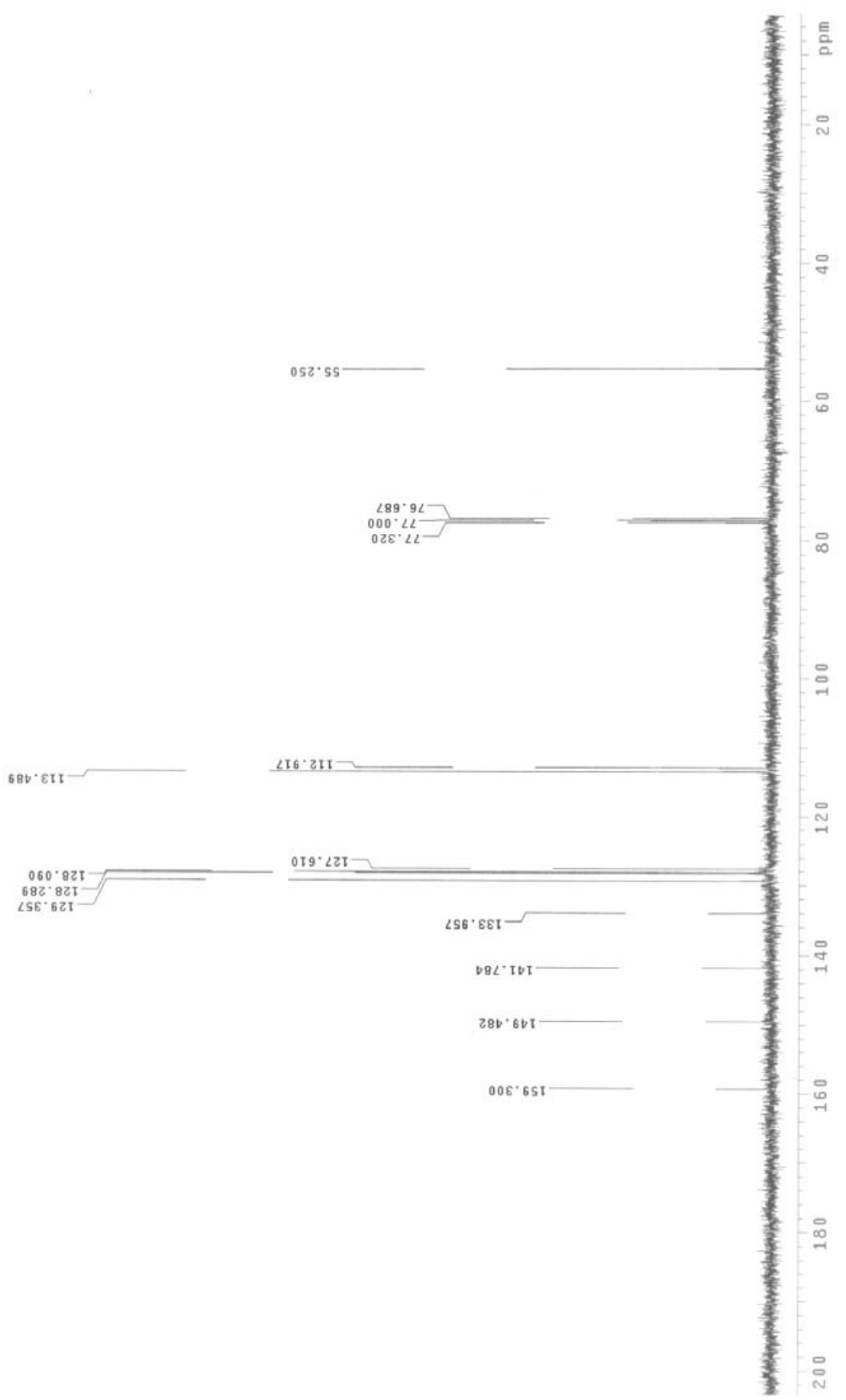


1-Fluoro-4-(1-phenylvinyl)benzene (3ed)

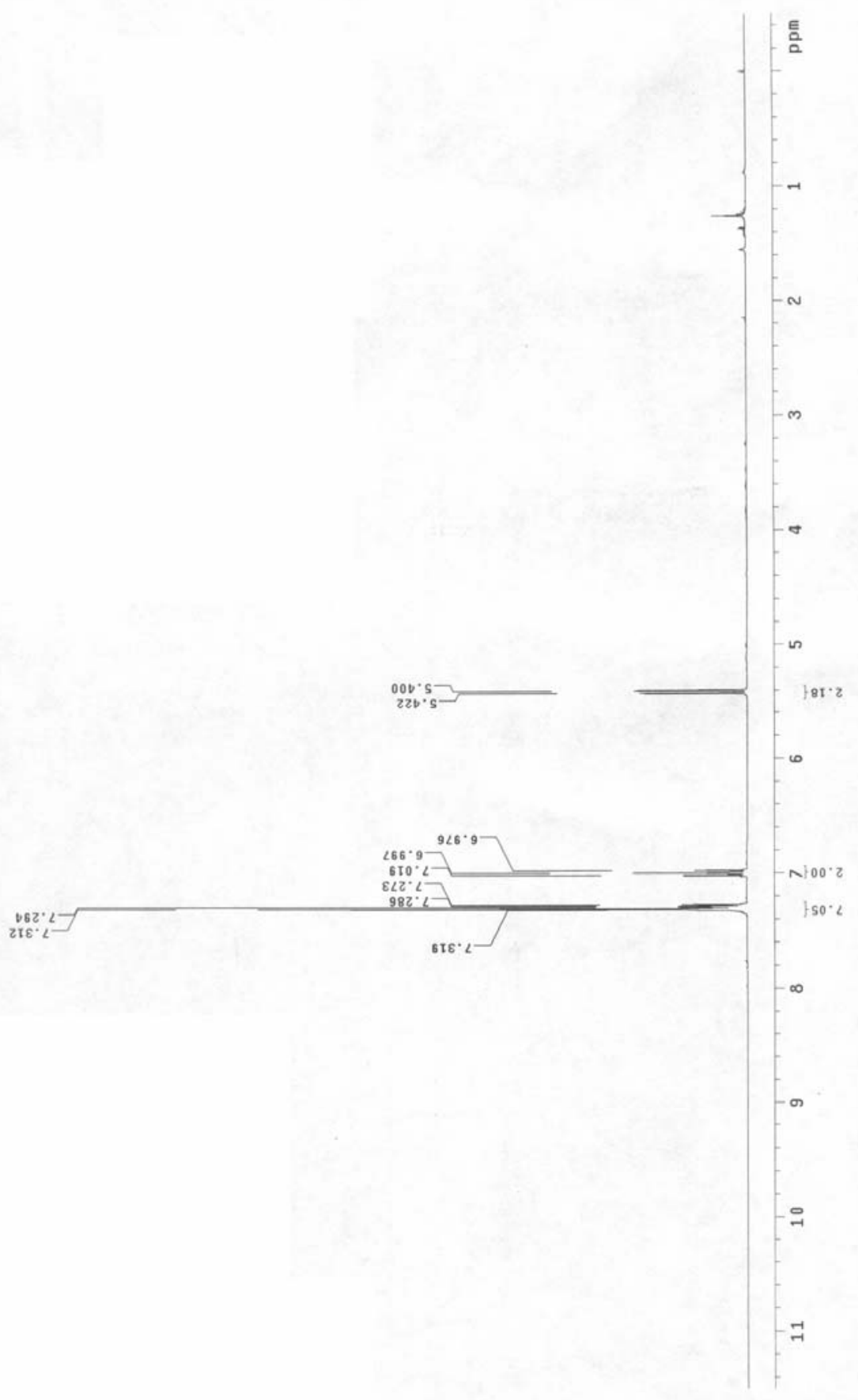


1-Fluoro-4-(1-phenylvinyl)benzene (3ed)

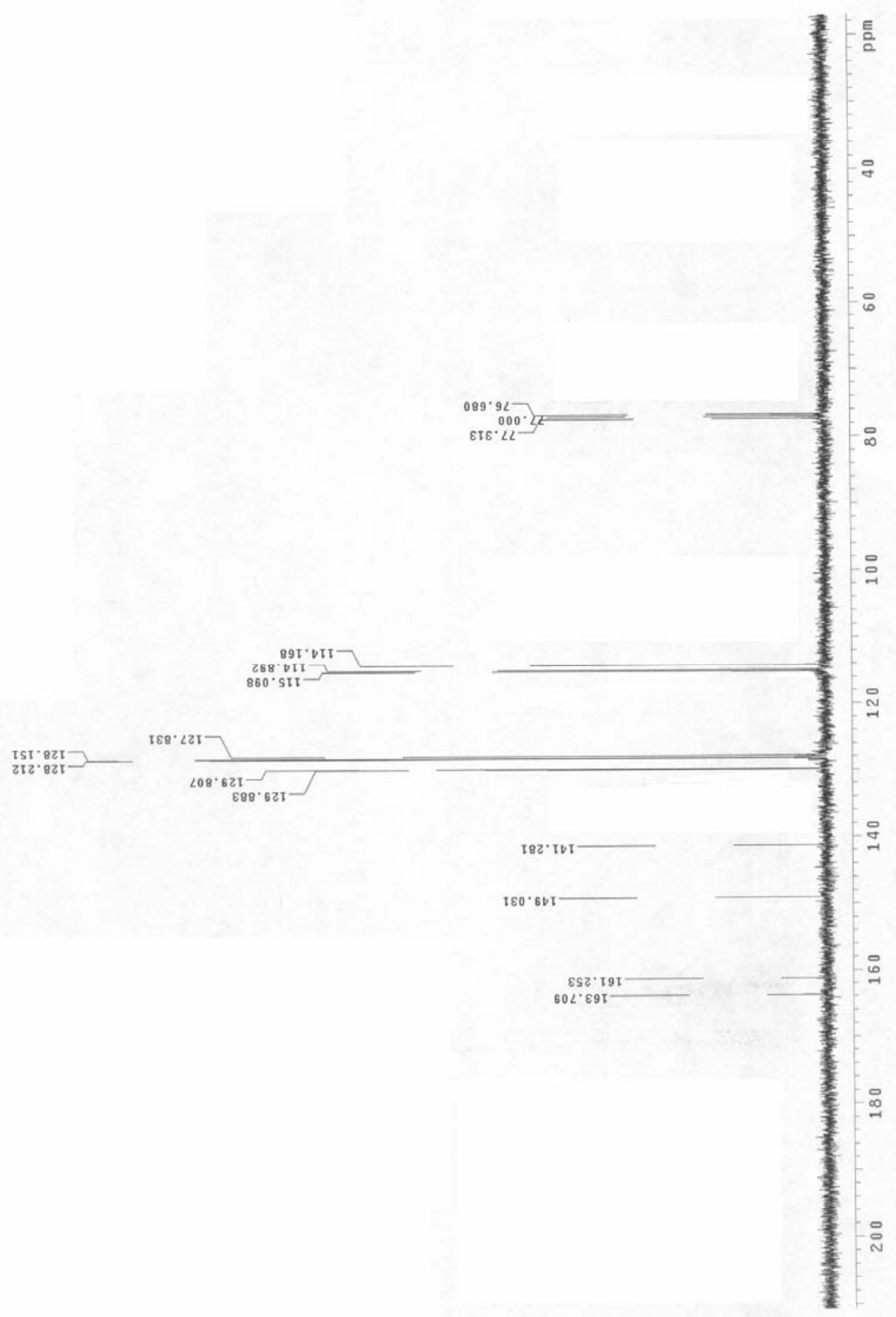


1,3-diphenylbenzene (3ga)

FFErg $\mathrm{F}$

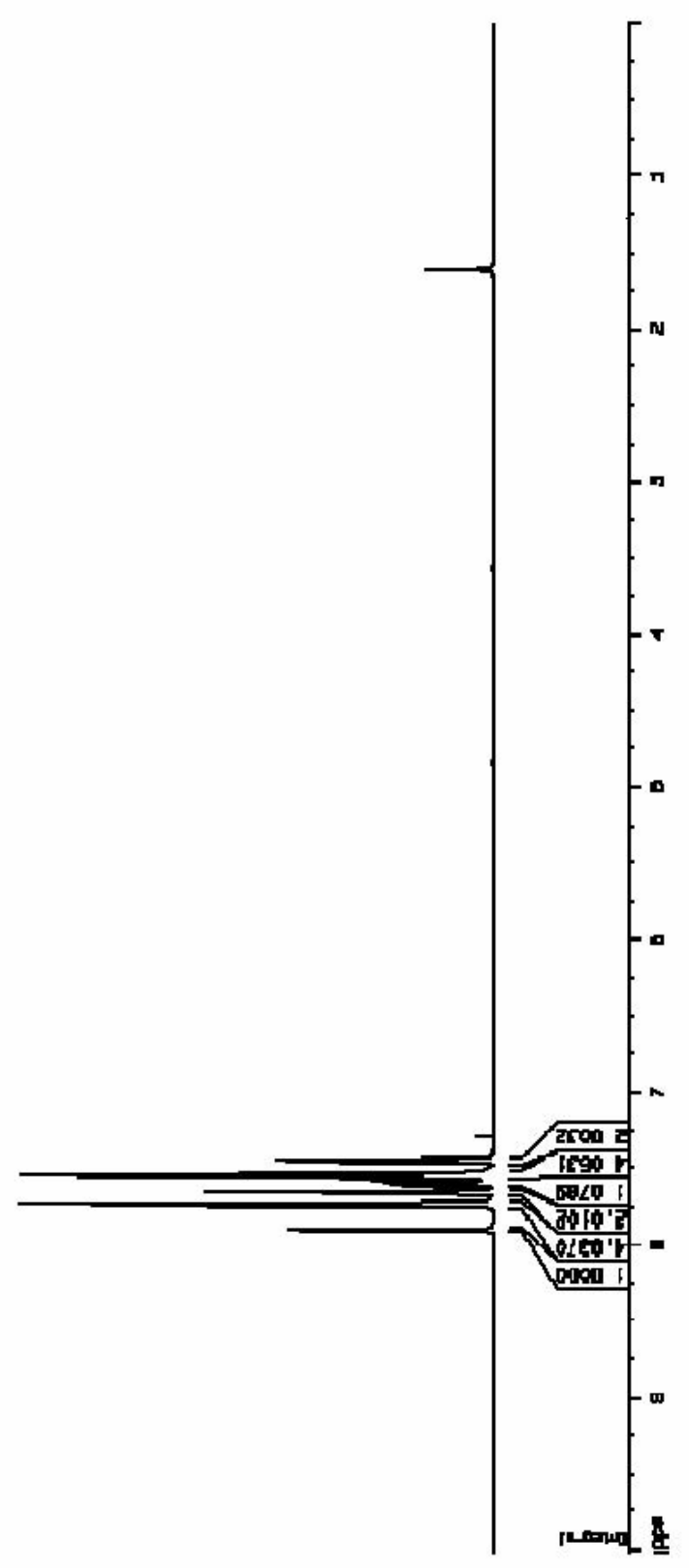

EIact $L$

Exit 47

또은

max 4

exrr 4

Dores $c-$

EStog $4-$

corsg

exorg' 2

coces $<-1$

osfer $2-$

eBrtc $<-3$

atre $c-$

aros' 1

vdd

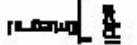


1,3-diphenylbenzene (3ga)

${ }_{i \in t} \| \longrightarrow>$

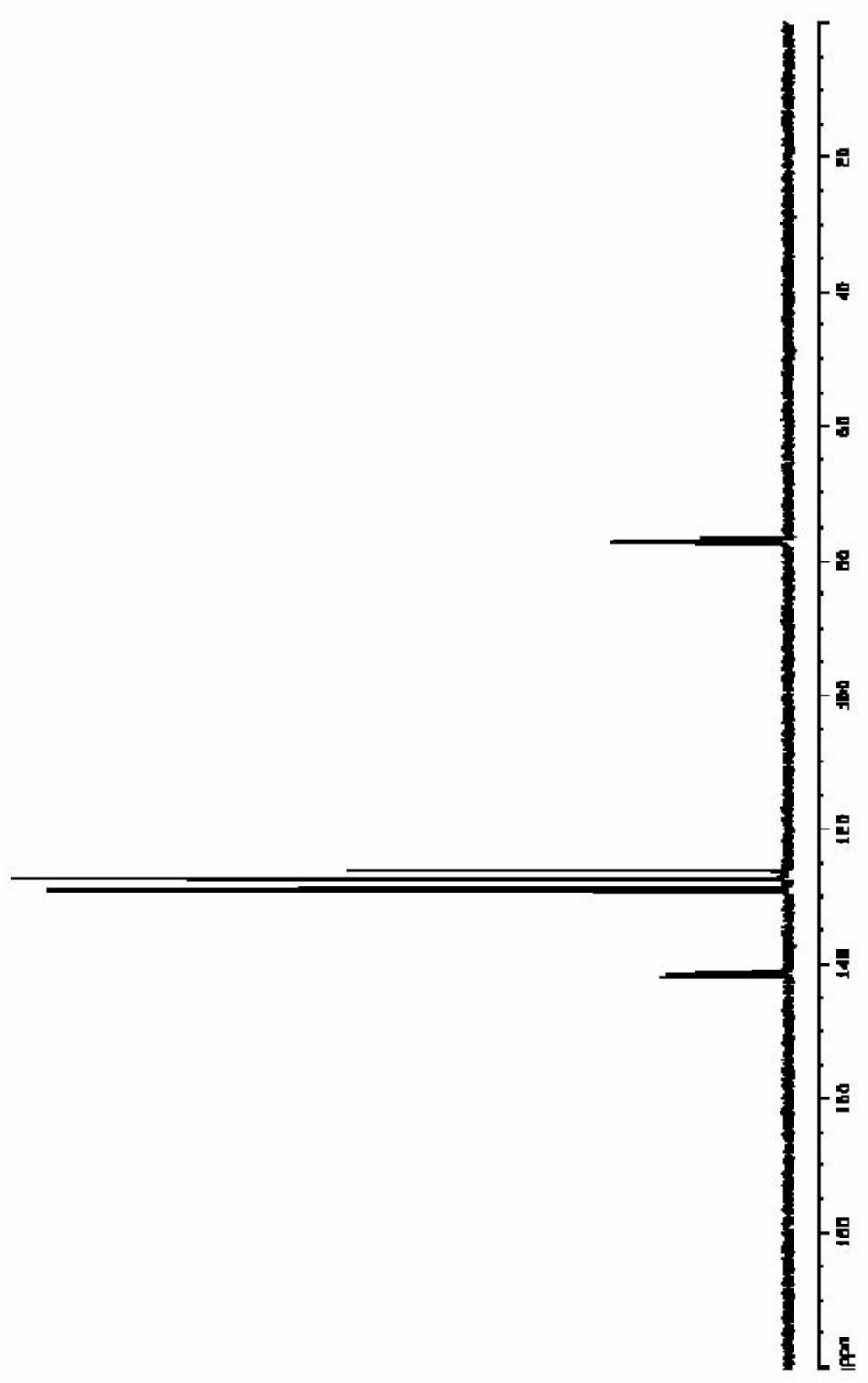


1,4-Diphenylbenzene (3ha)

LCRO [-

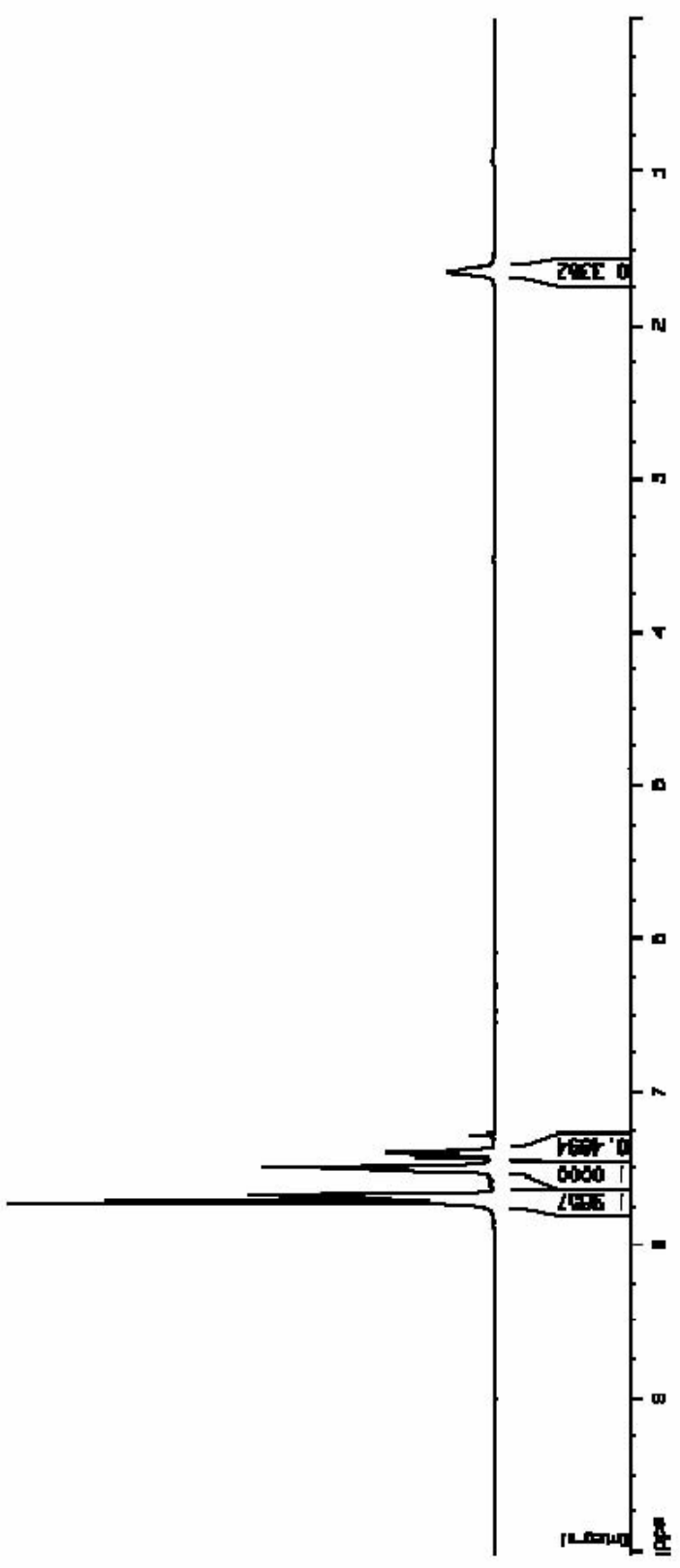

mesec's

c981': $[-$

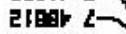

Bezod 4

ए人il $<$

coos 4

Eif69 $1-$

Ex地 ' $L$

vad

(L) 
1,4-diphenylbenzene (3ha)

act $\underline{\mathbf{y}}$

जis

LWO IEC

rat der-

나 te든

Ef opr

cet qpr

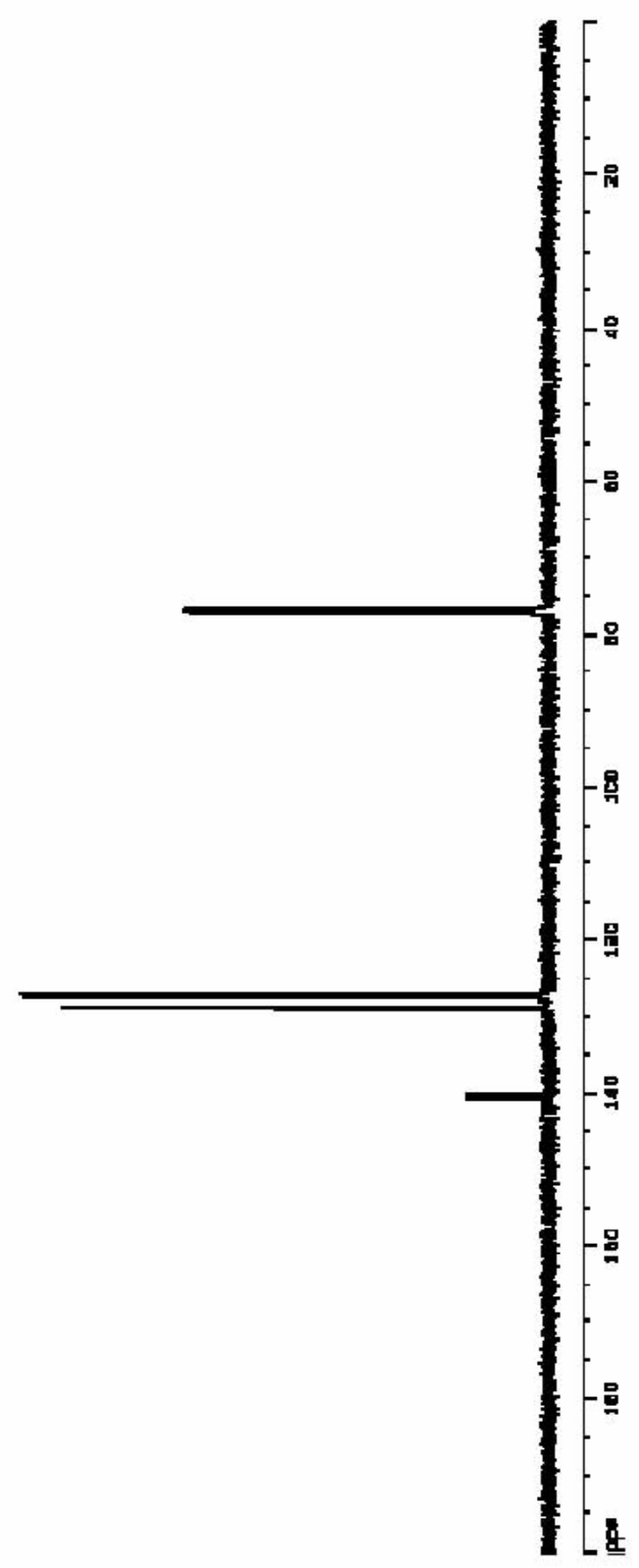

udd 
1,3,5-triphenylbenzene (3ia)
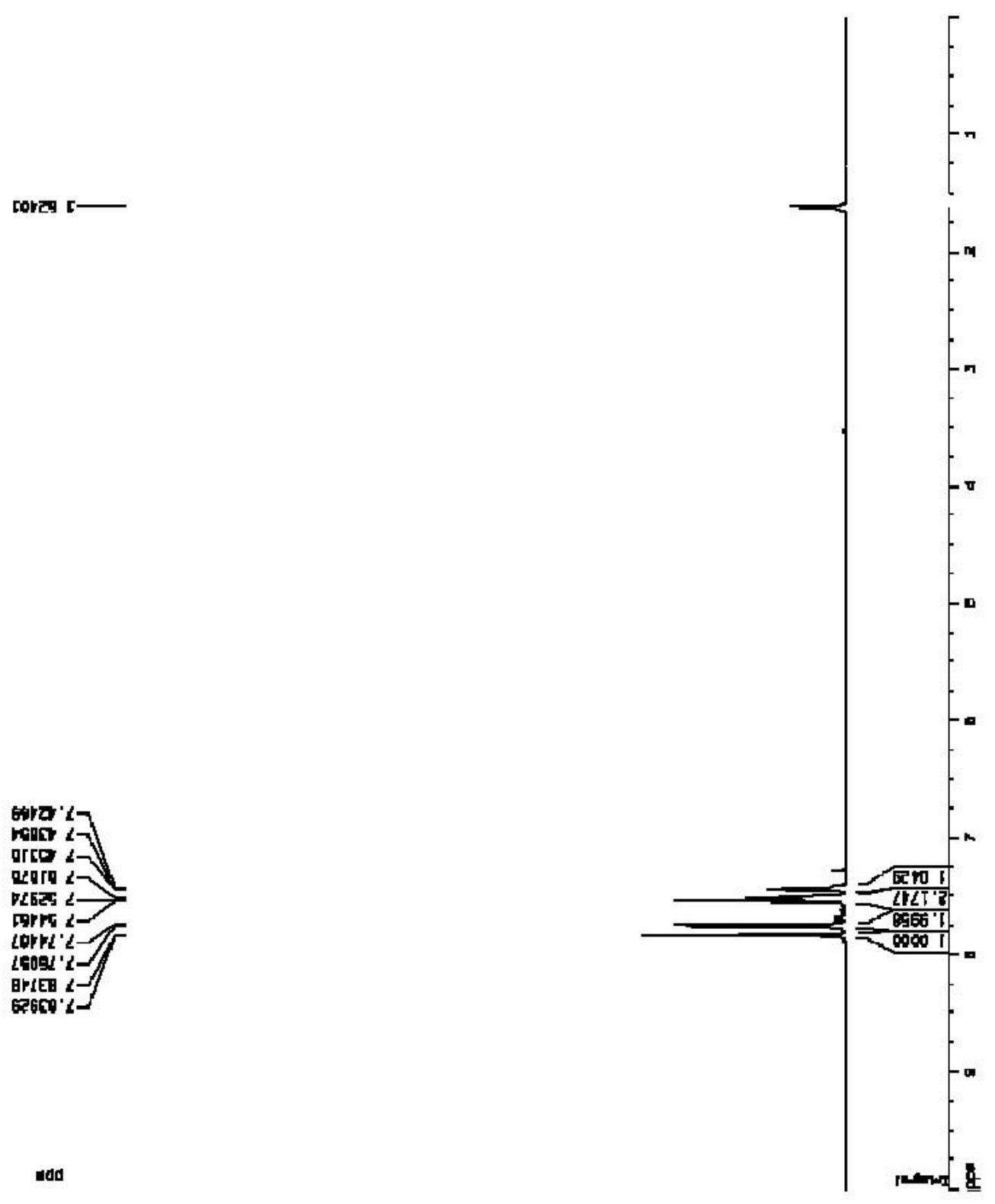
1,3,5-triphenylbenzene (3ia)

다 $n$

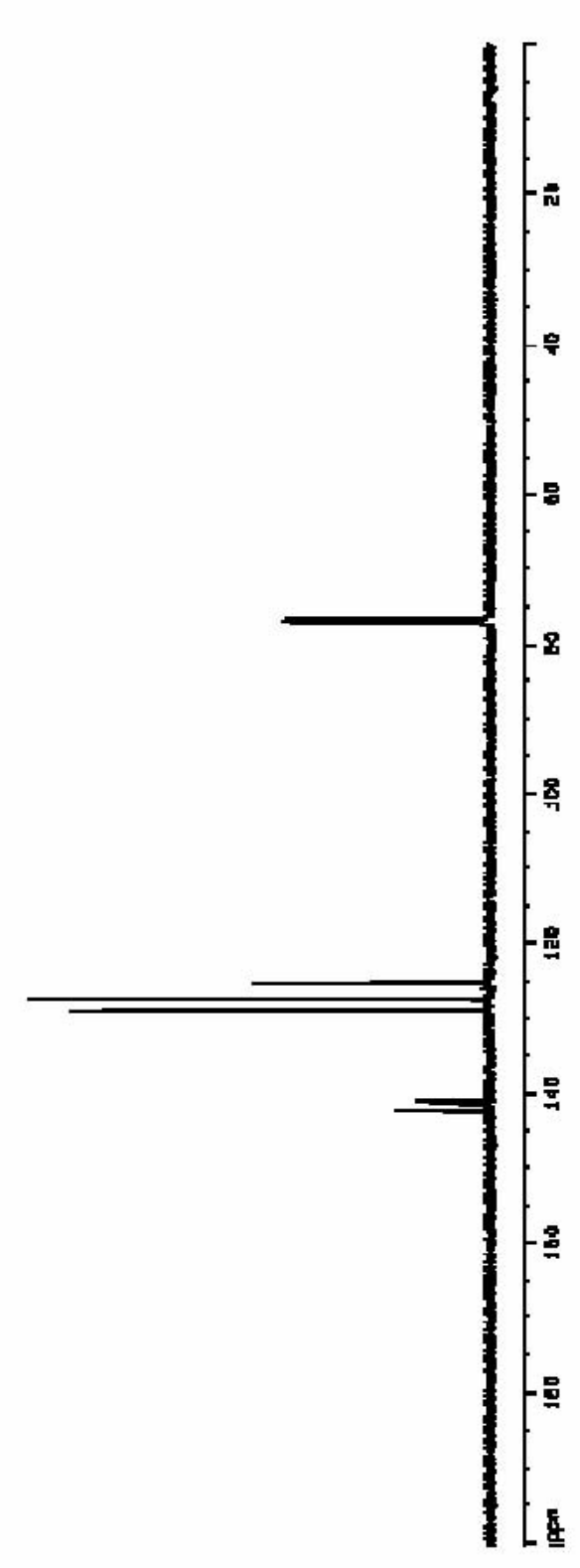

더비

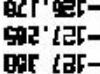

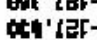

हEI $\mathrm{ger}-$

[te' ' Пh:

chis $12=$ 
(E)-1,4-Diphenylbut-1-en-3-yne (5aa)

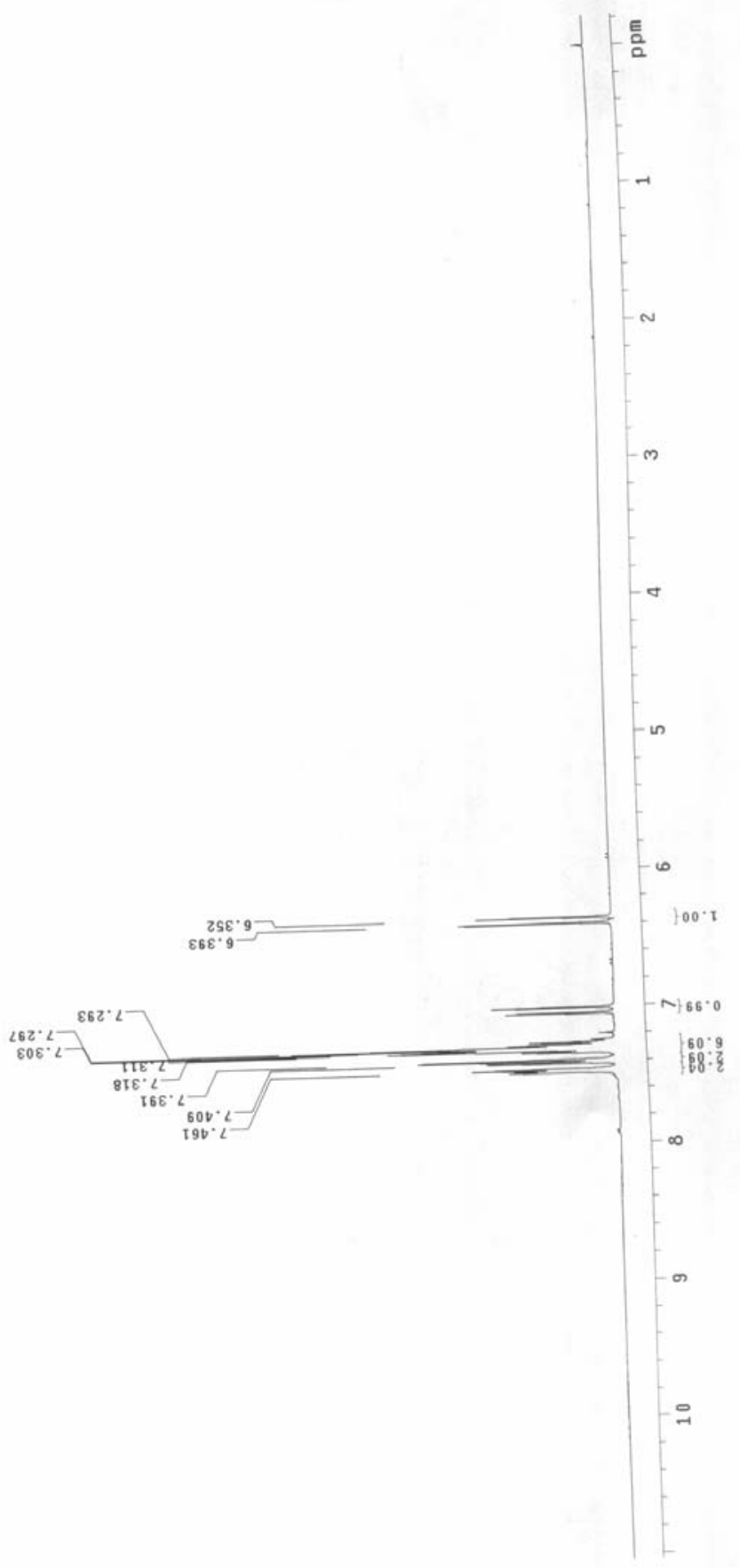


(E)-1,4-Diphenylbut-1-en-3-yne (5aa)

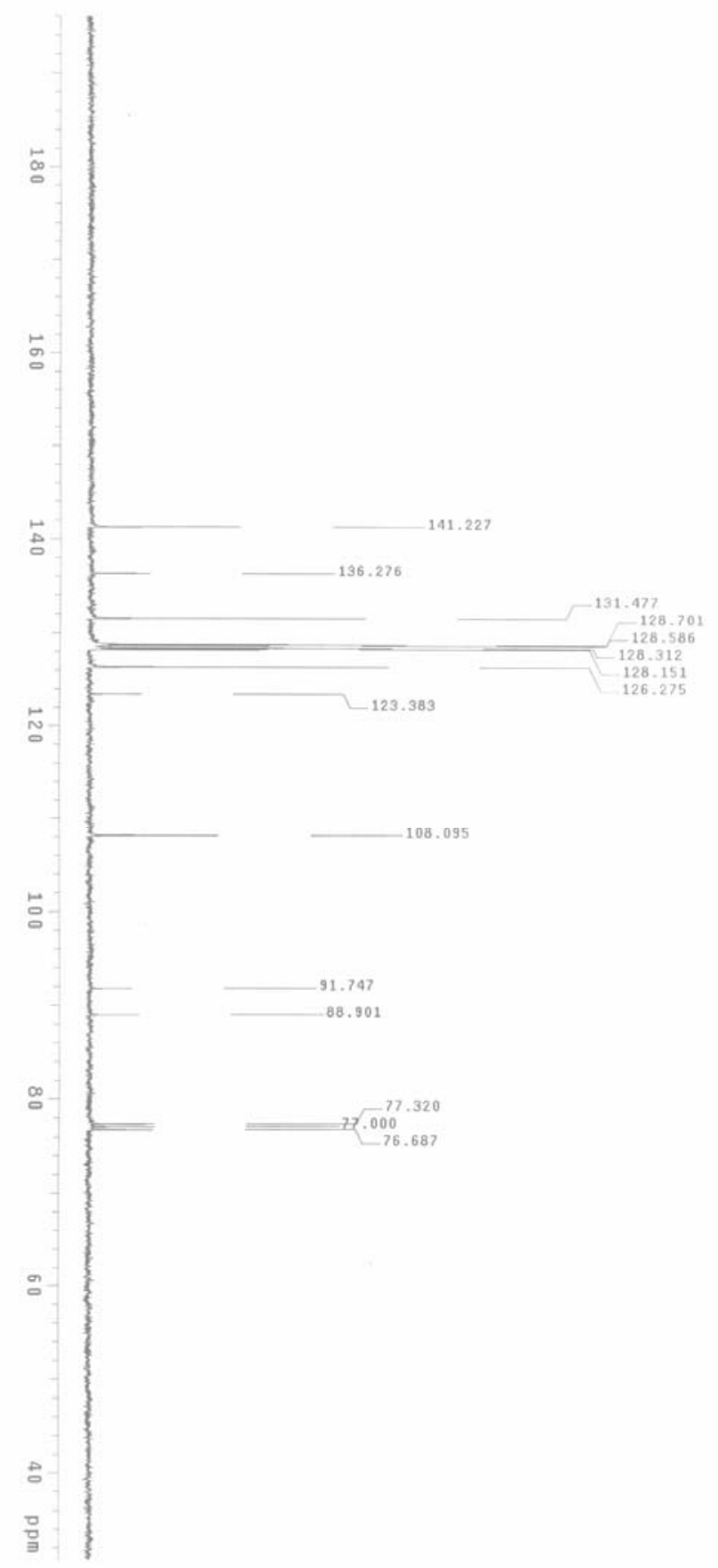


(E)-1-Chloro-4-(4-phenylbut-1-en-3-ynyl)benzene (5ba)

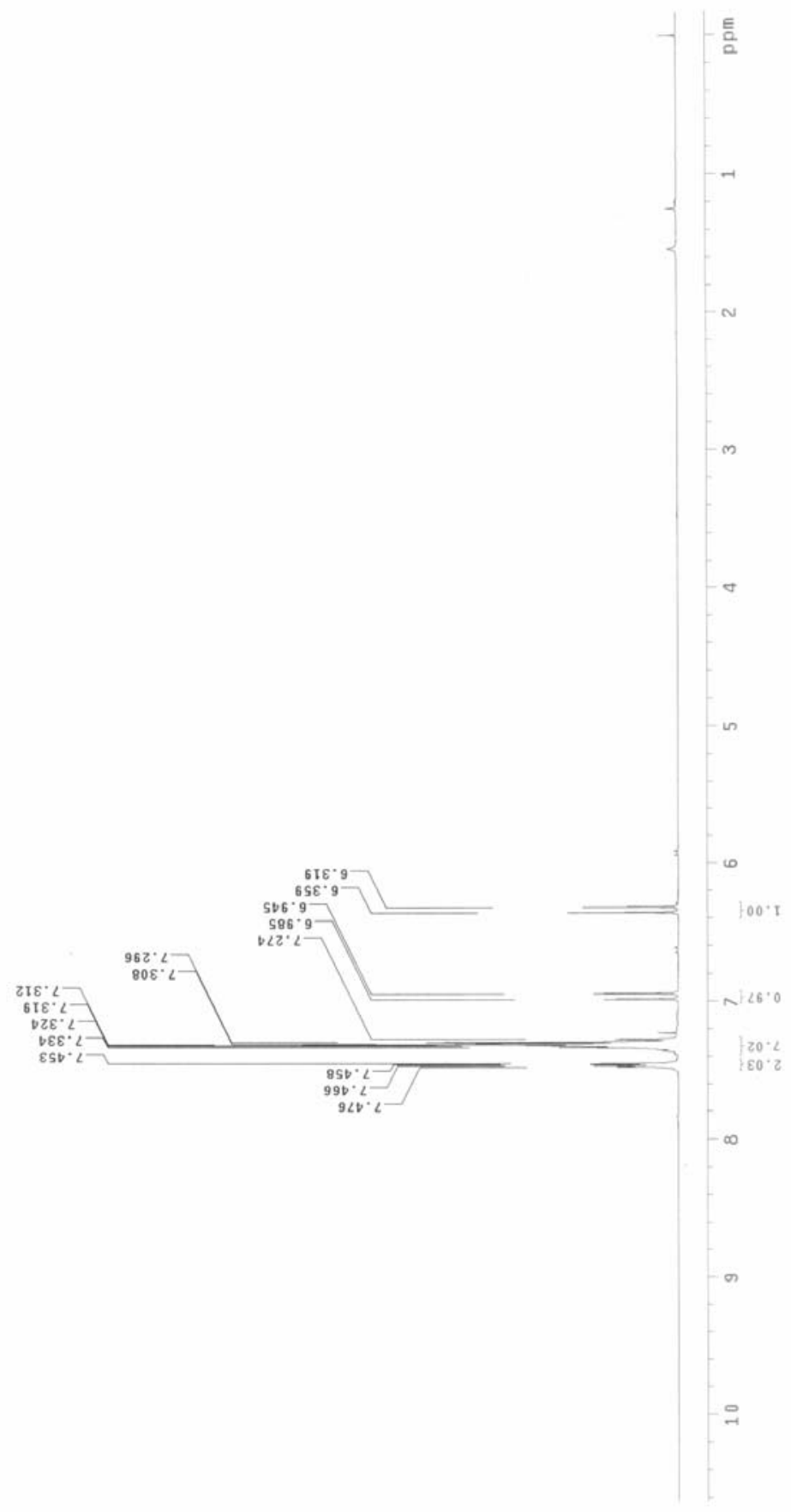


(E)-1-Chloro-4-(4-phenylbut-1-en-3-ynyl)benzene (5ba)

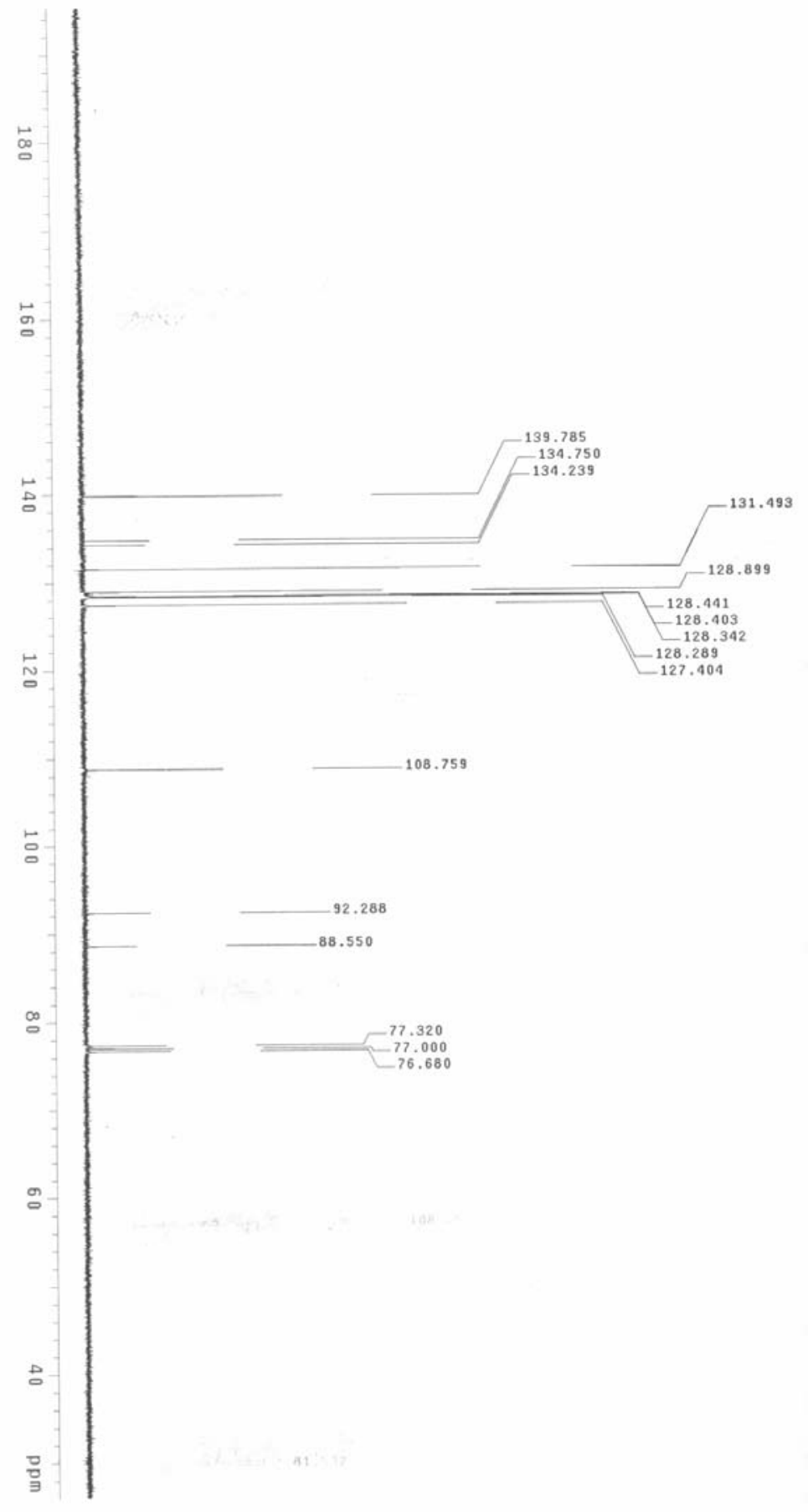


(E)-1-(Non-1-en-3-ynyl)benzene (5db)

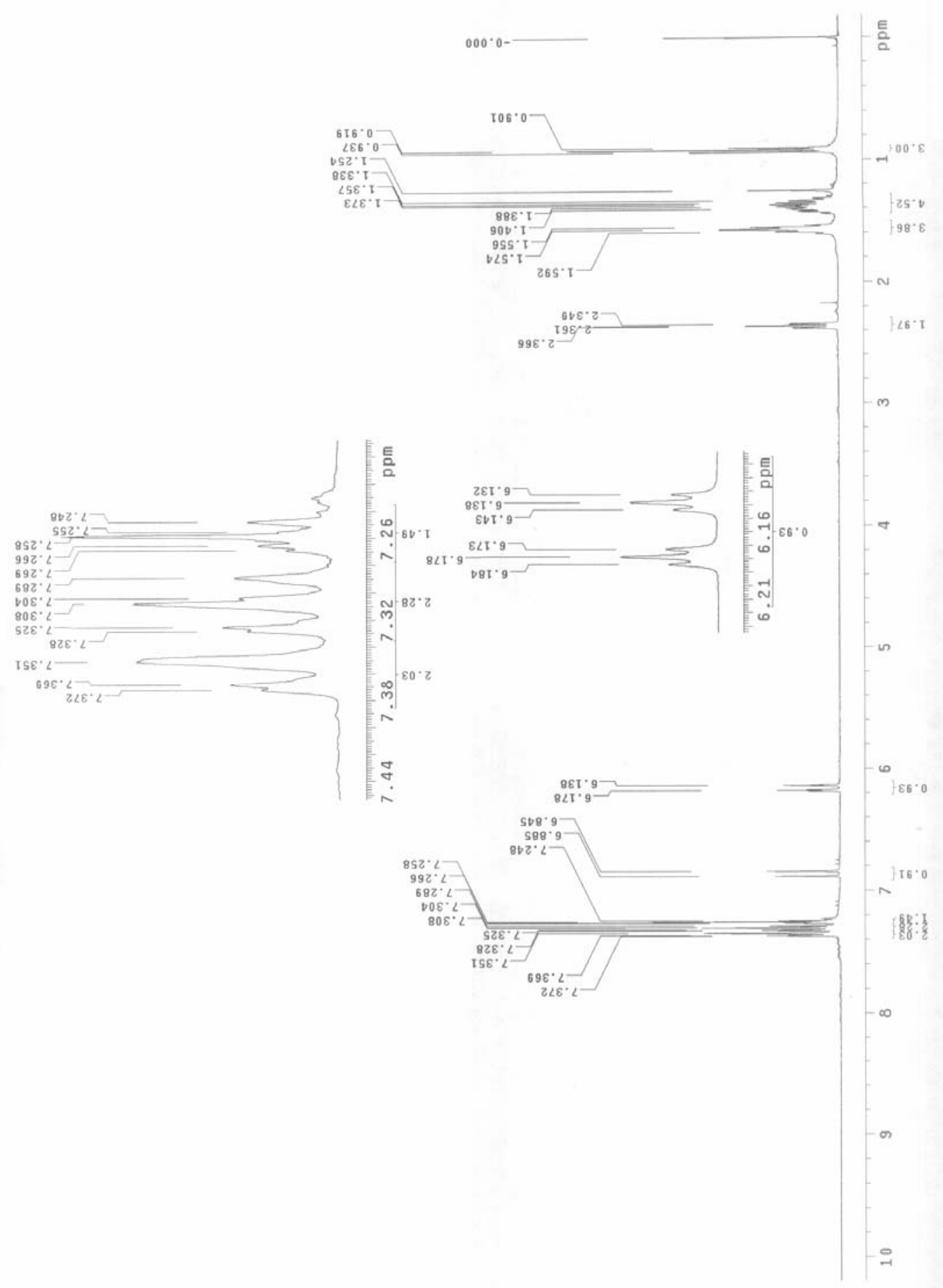


(E)-1-(Non-1-en-3-ynyl)benzene (5db)

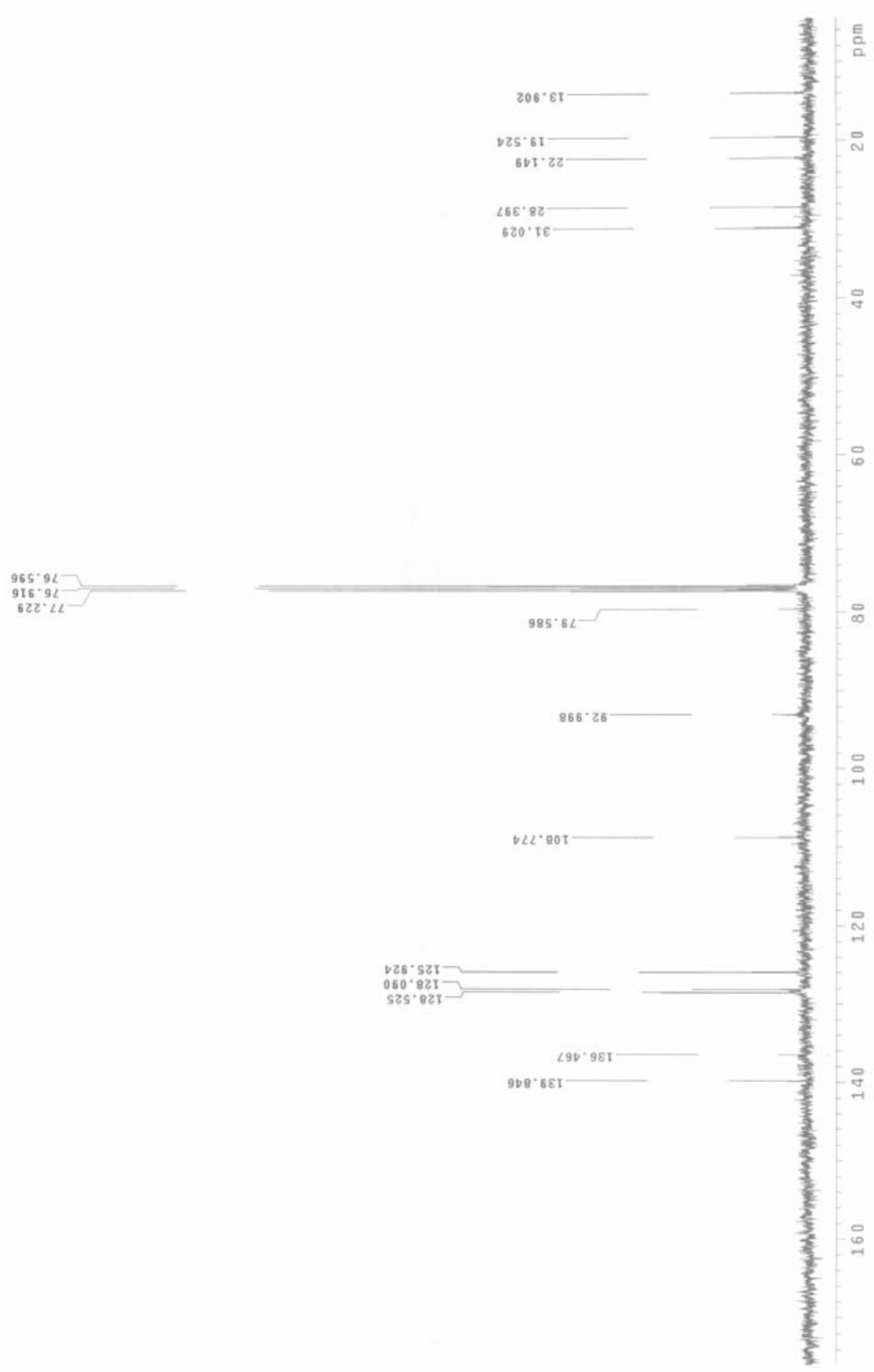


1-Methoxy-4-(2-phenylethynyl)benzene (5ja)

$20000^{\circ} 0$

2ESO5. -

$\operatorname{EDBCB} \mathrm{C}^{\circ} \mathrm{-}$

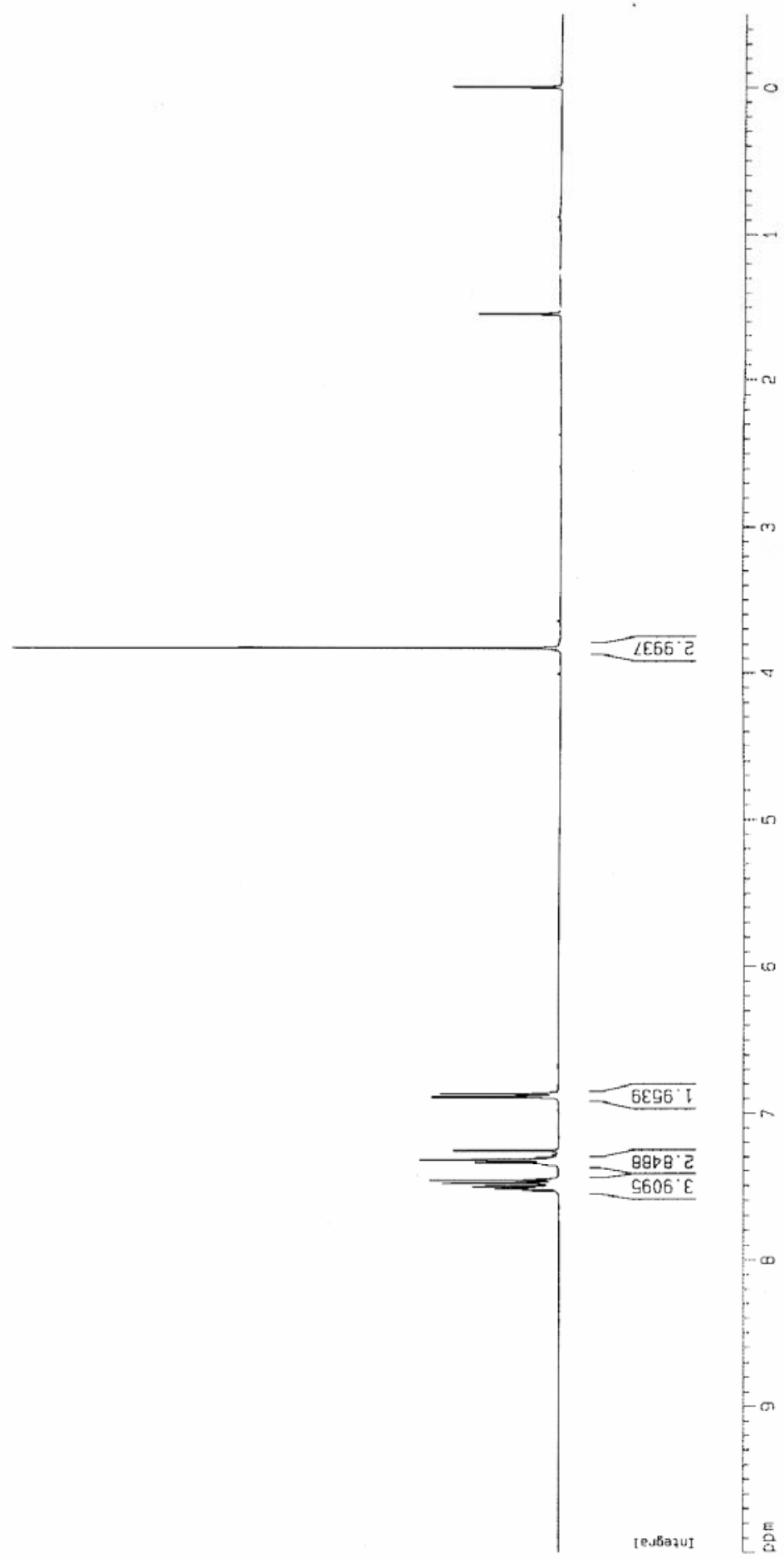

$90 \angle 98^{\circ} 9-$

ᄂ688. 9

06952 L

6I8IE. $\angle$

9G2टE' $\angle-$

О૪ $9 E \varepsilon^{\prime}<-$

D2090 $\angle$

$\mathrm{EVC80^{ \circ } \angle -}$

$\triangle 6860^{\circ} L$

$6 \angle 209^{\circ} \angle$

969052

IB9.5' $\angle$

$16 \angle 1 \mathrm{~S}^{\circ} \angle$

$90229^{\circ} L$

wdd 
1-methoxy-4-(2-phenylethynyl)benzene (5ja)

$\angle B C \cdot 99$

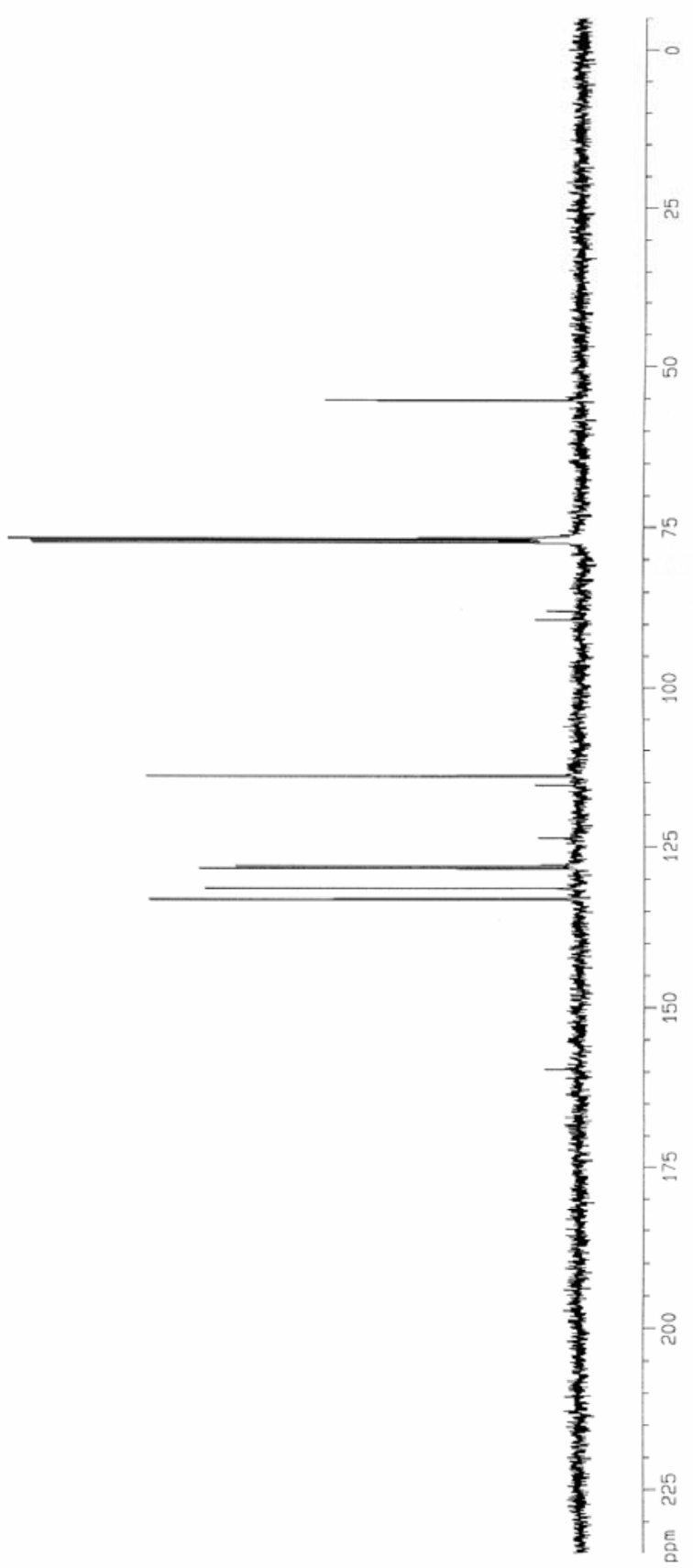


1-(dec-1-ynyl)-4-methoxybenzene (5jb)

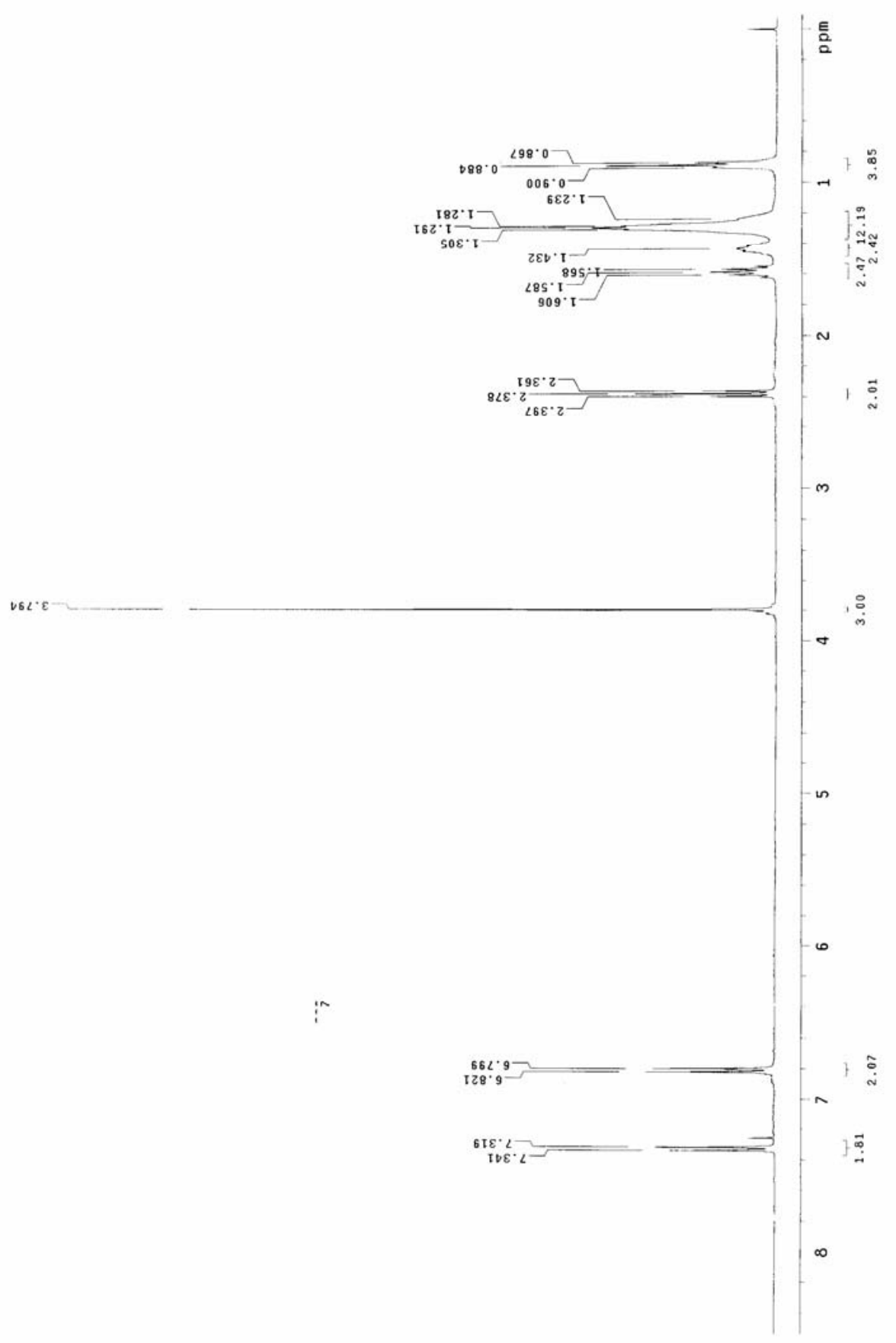


1-(dec-1-ynyl)-4-methoxybenzene (5jb)

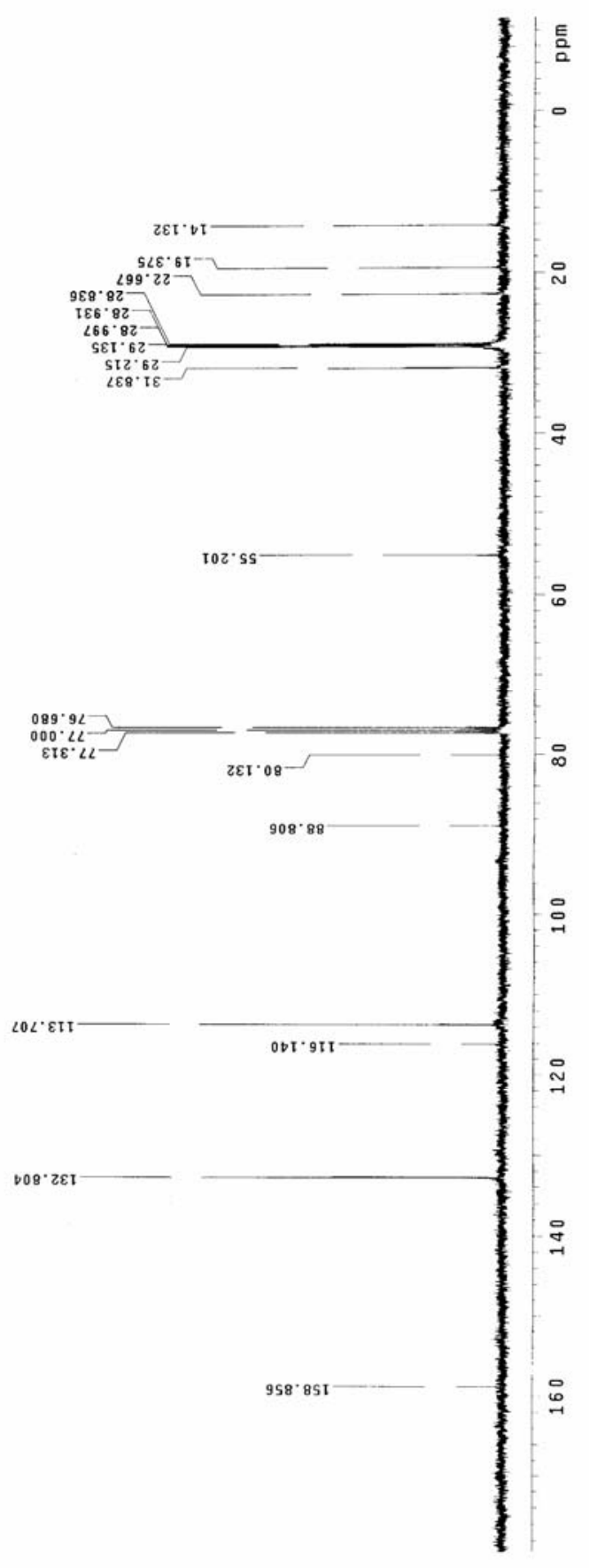


2-(3-(4-Methoxyphenyl)prop-2-ynyloxy)-tetrahydro-2H-pyran (5jc)

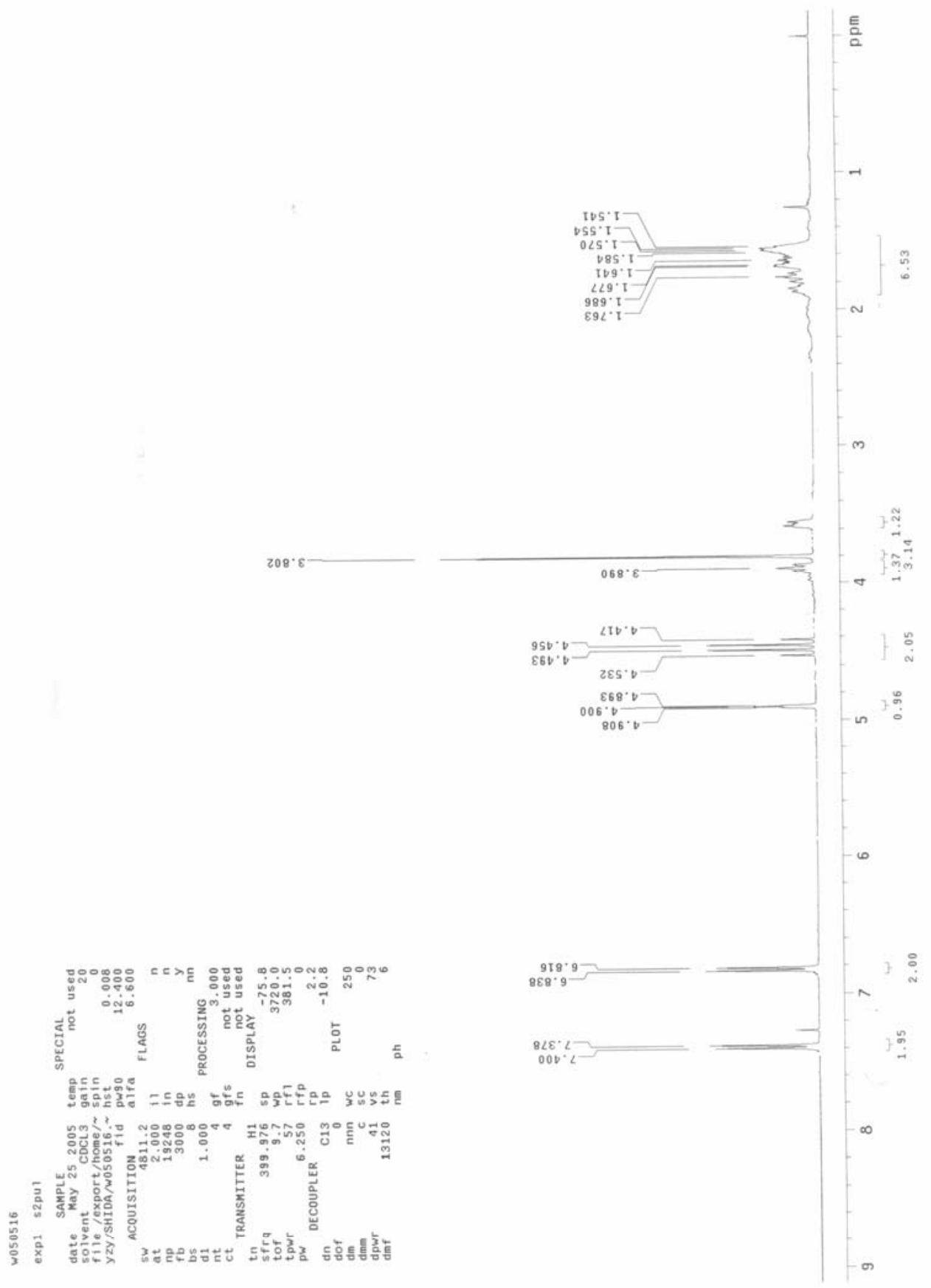


2-(3-(4-Methoxyphenyl)prop-2-ynyloxy)-tetrahydro-2H-pyran (5jc)

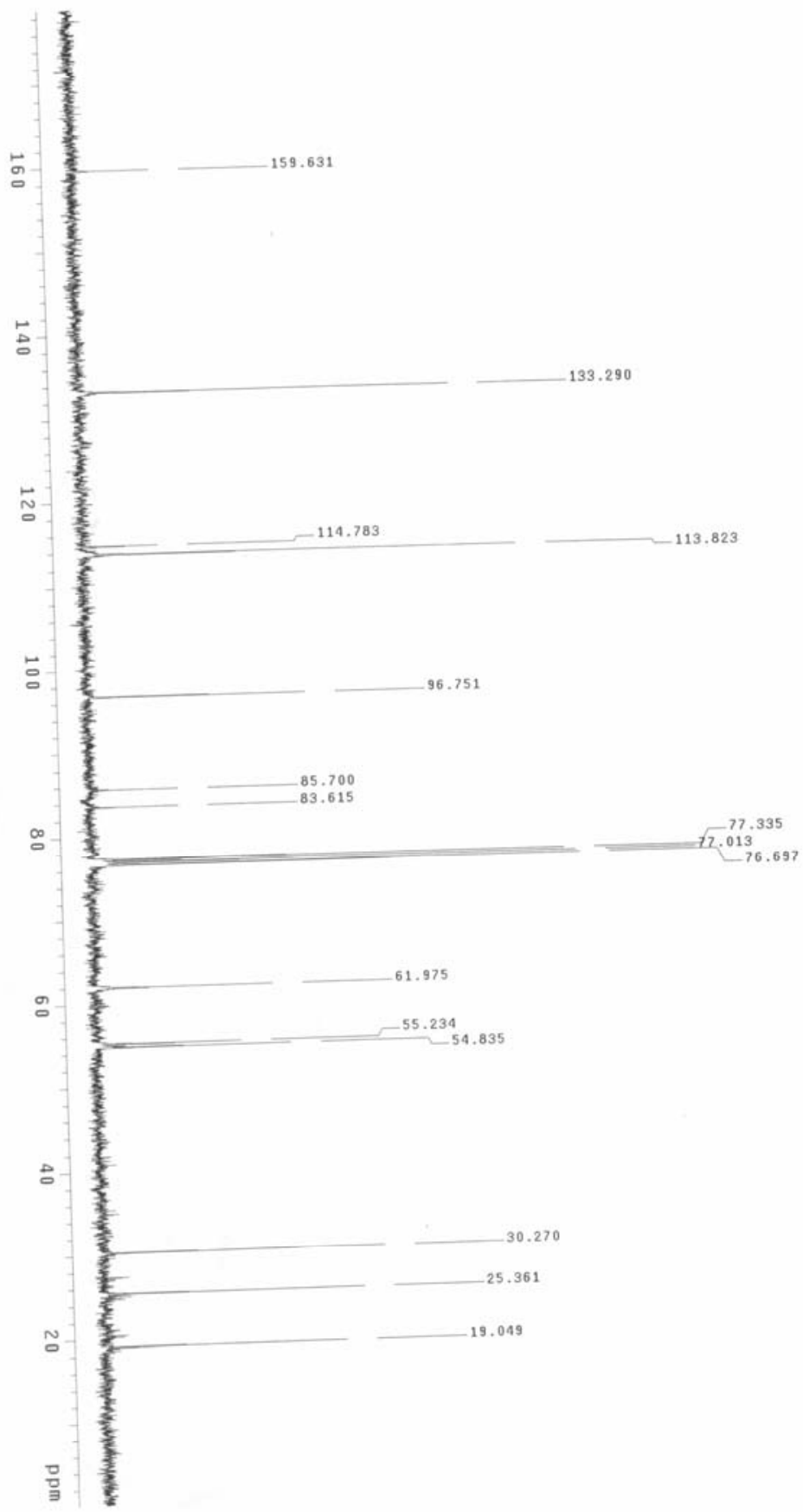


1-(2-(4-nitrophenyl)ethynyl)benzene (5ka)
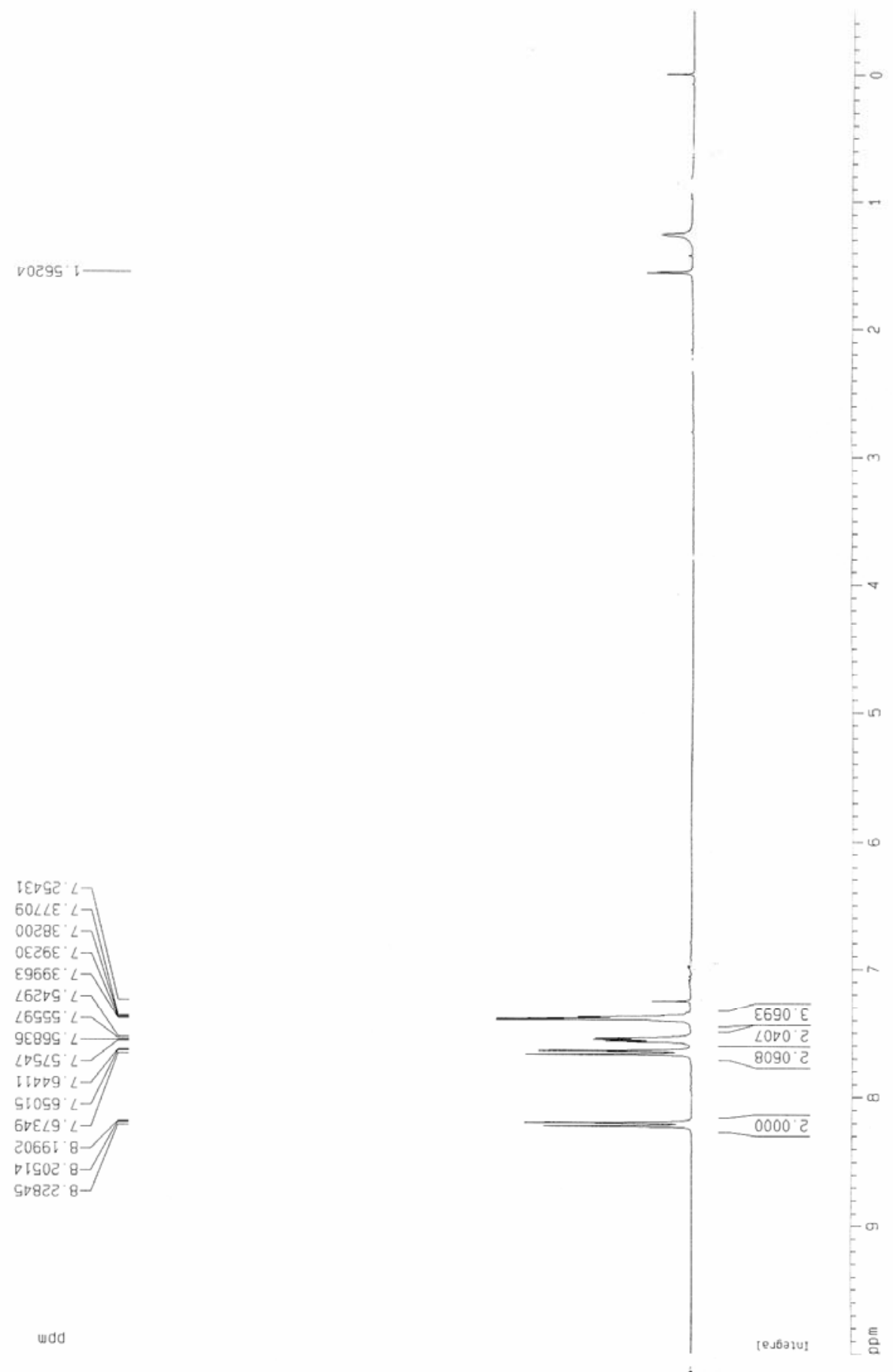
1-(2-(4-nitrophenyl)ethynyl)benzene (5ka)
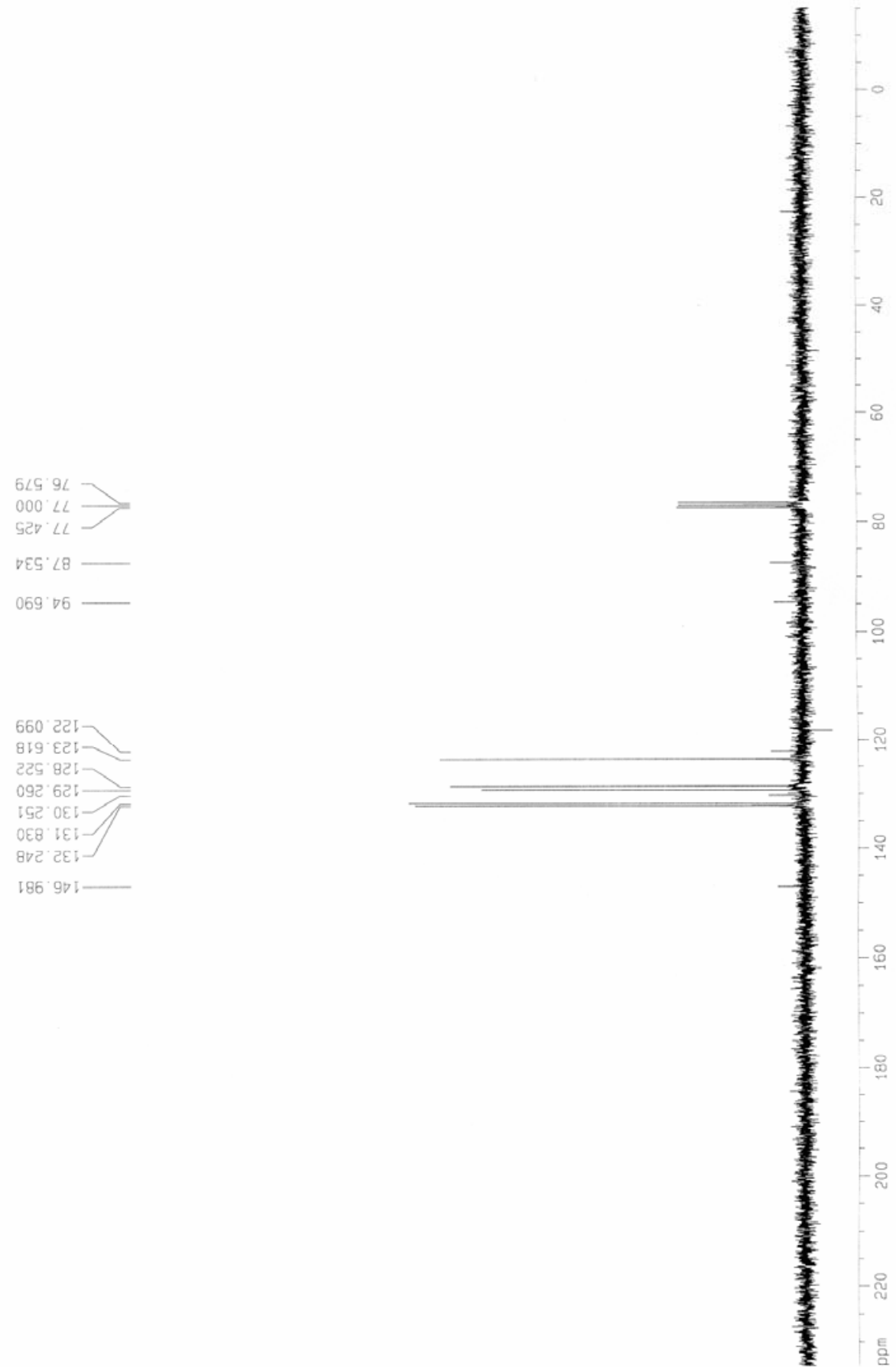
1-(dec-1-ynyl)-4-nitrobenzene (5kb)
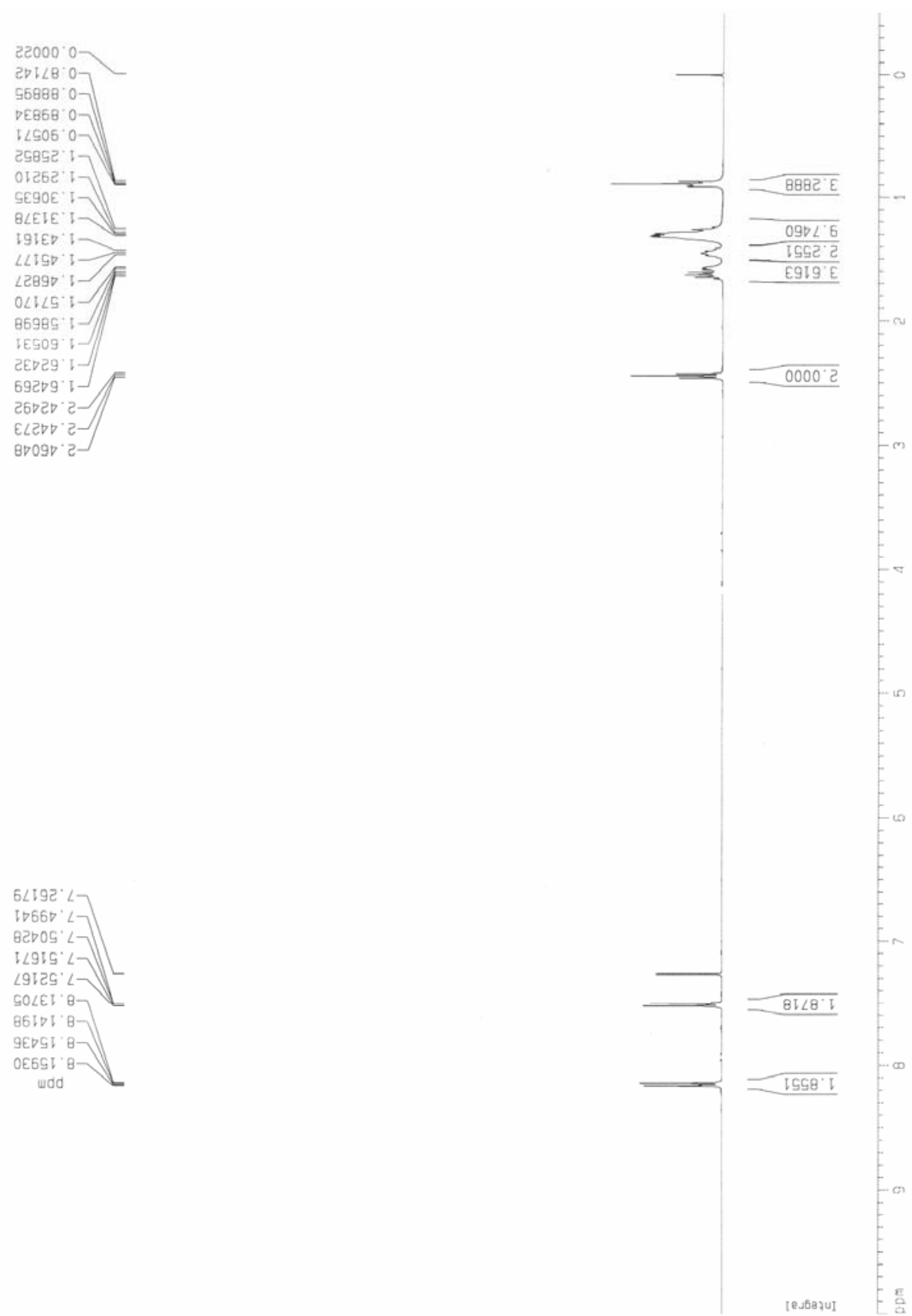
1-(dec-1-ynyl)-4-nitrobenzene (5kb)
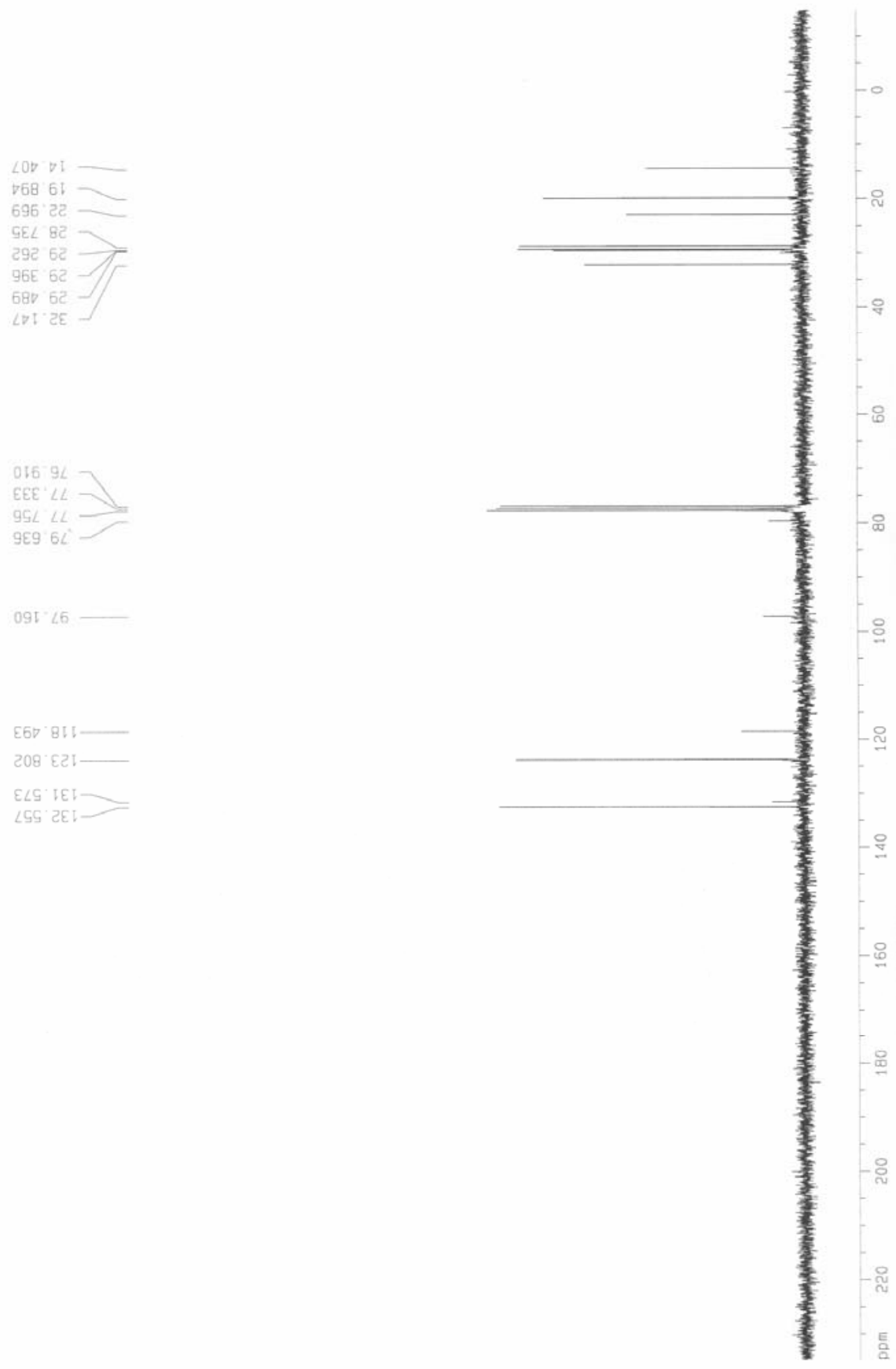


\section{1-(2-(2-nitrophenyl)ethynyl)benzene (5la)}

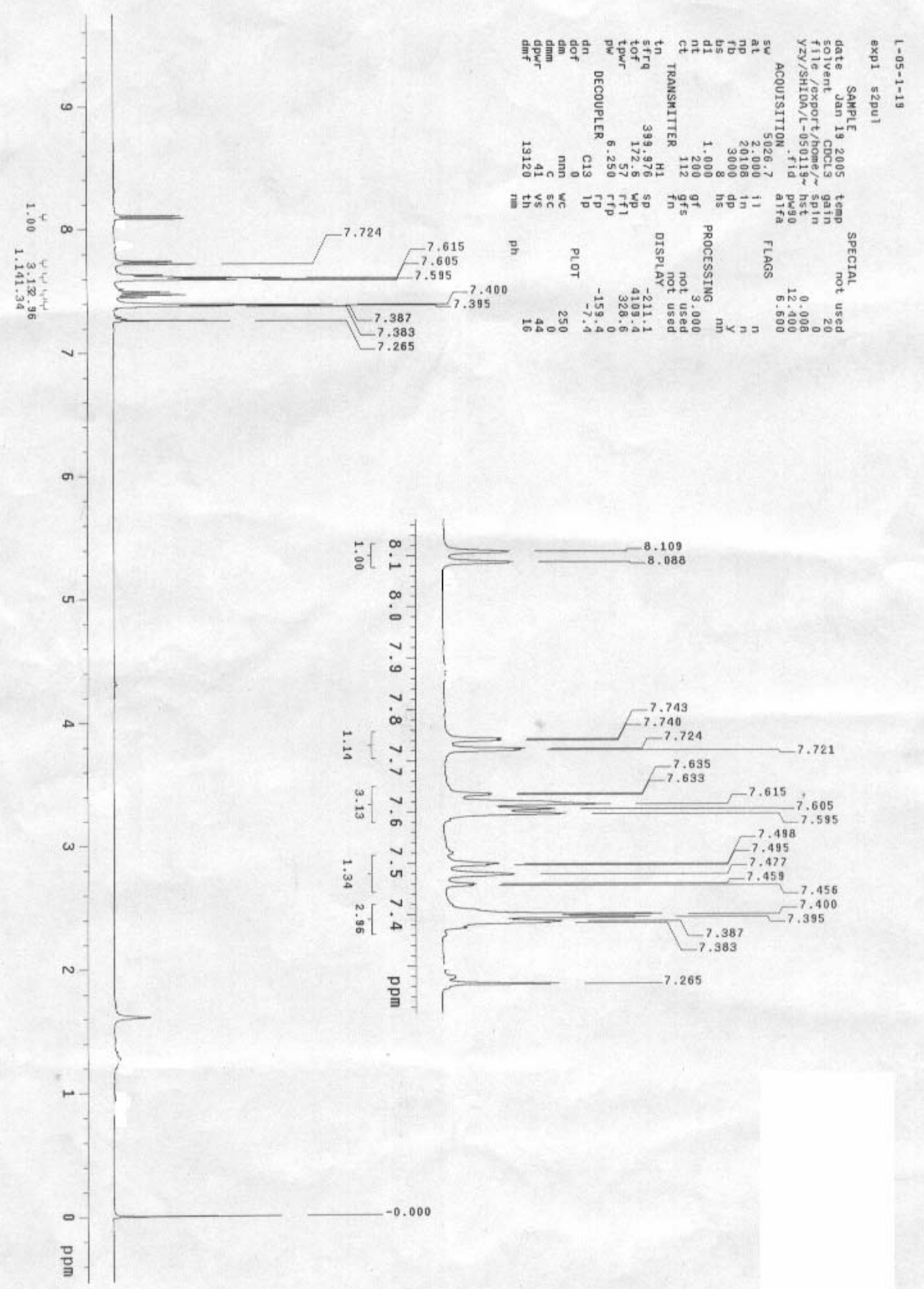


1-(2-(2-nitrophenyl)ethynyl)benzene (5la)

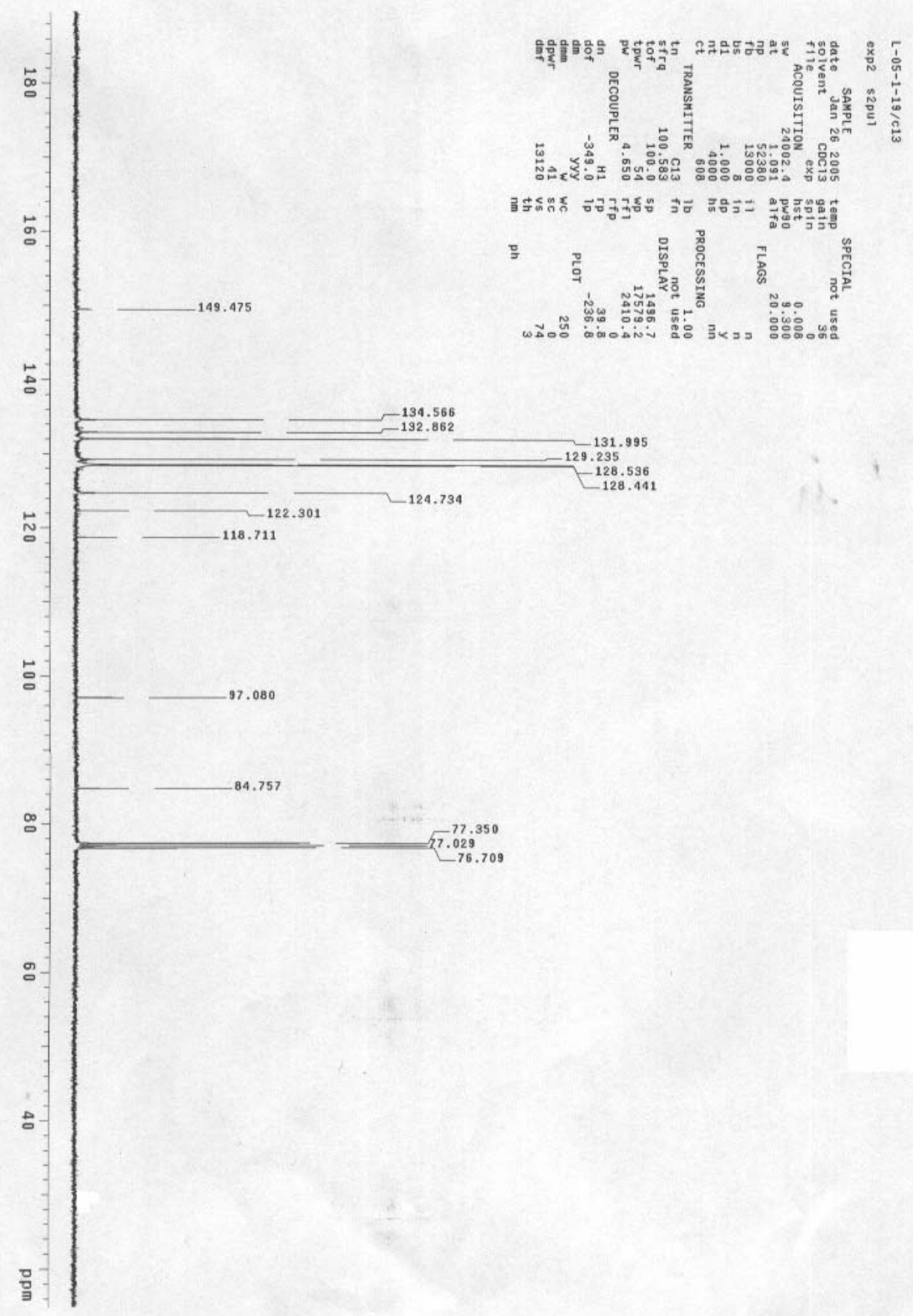


1,2-diphenylethyn (5ma)

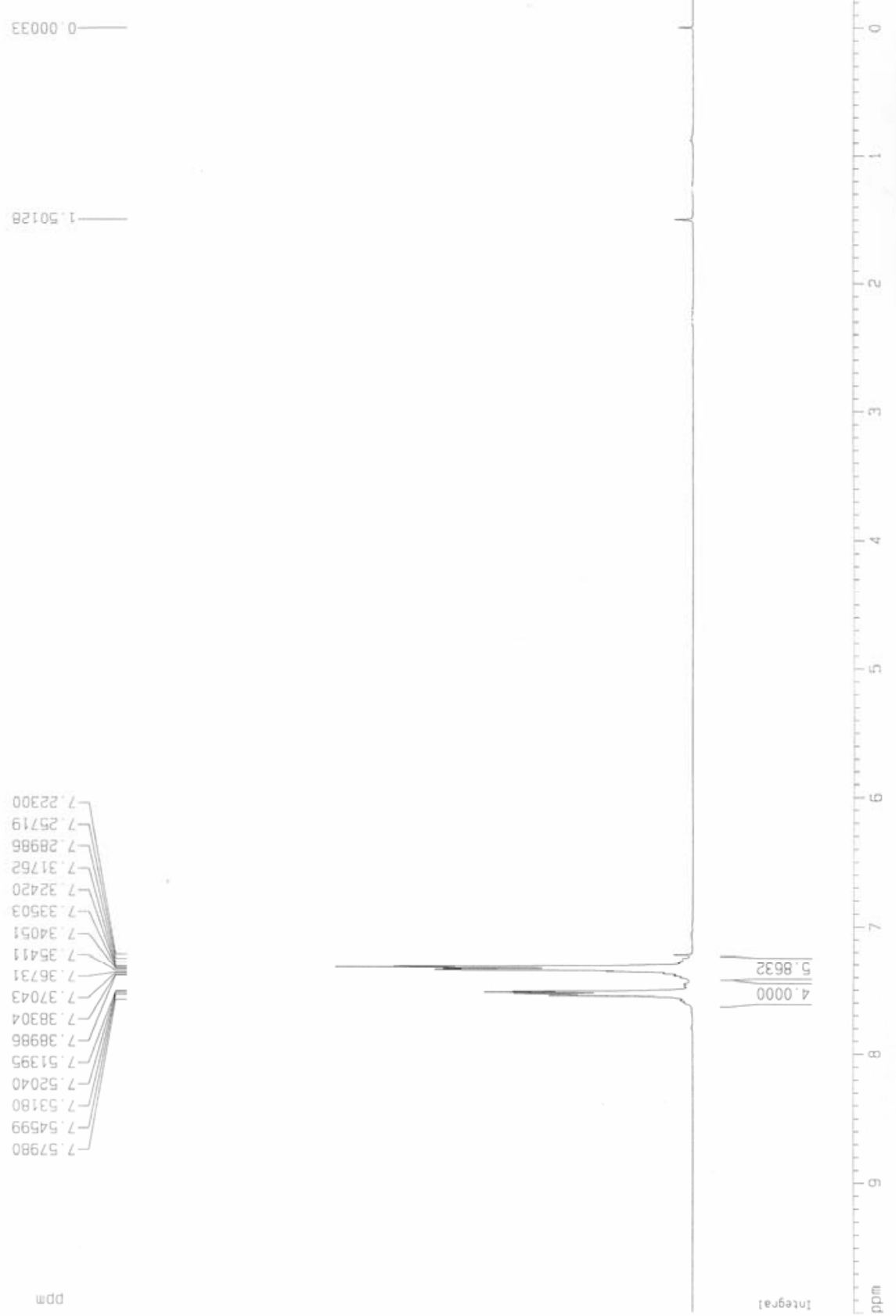


1,2-diphenylethyn (5ma)

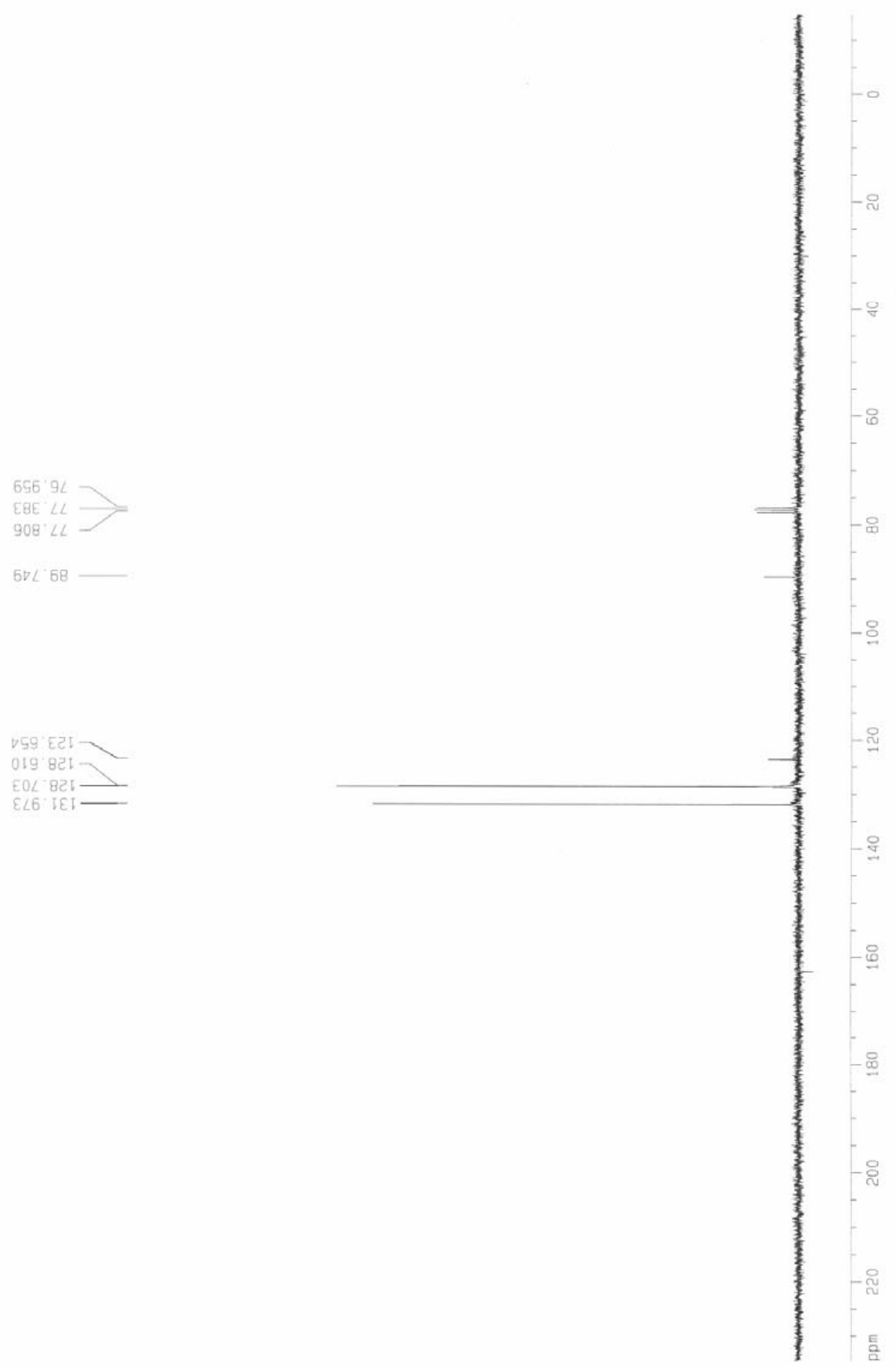


1-(2-p-tolylethynyl)benzene (5na)

$20000-$

OLटद :

ट6SE 2

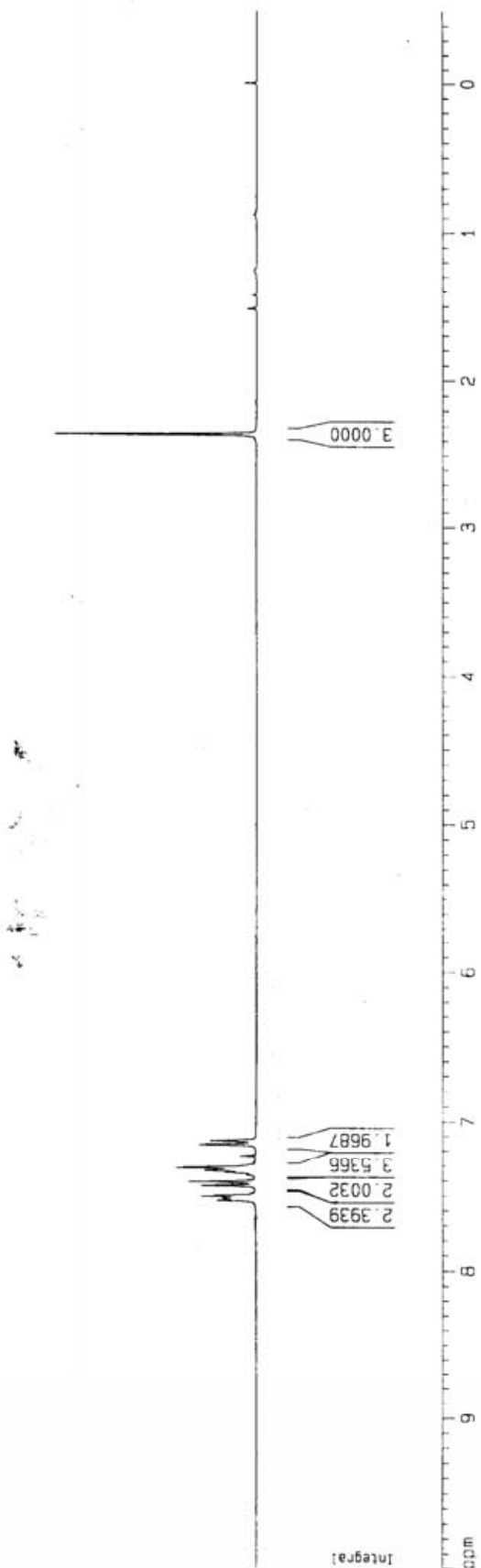

wad

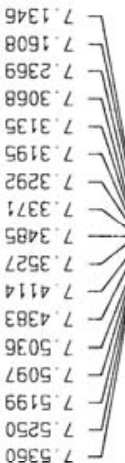


1-(2-p-tolylethynyl)benzene (5na)

19812
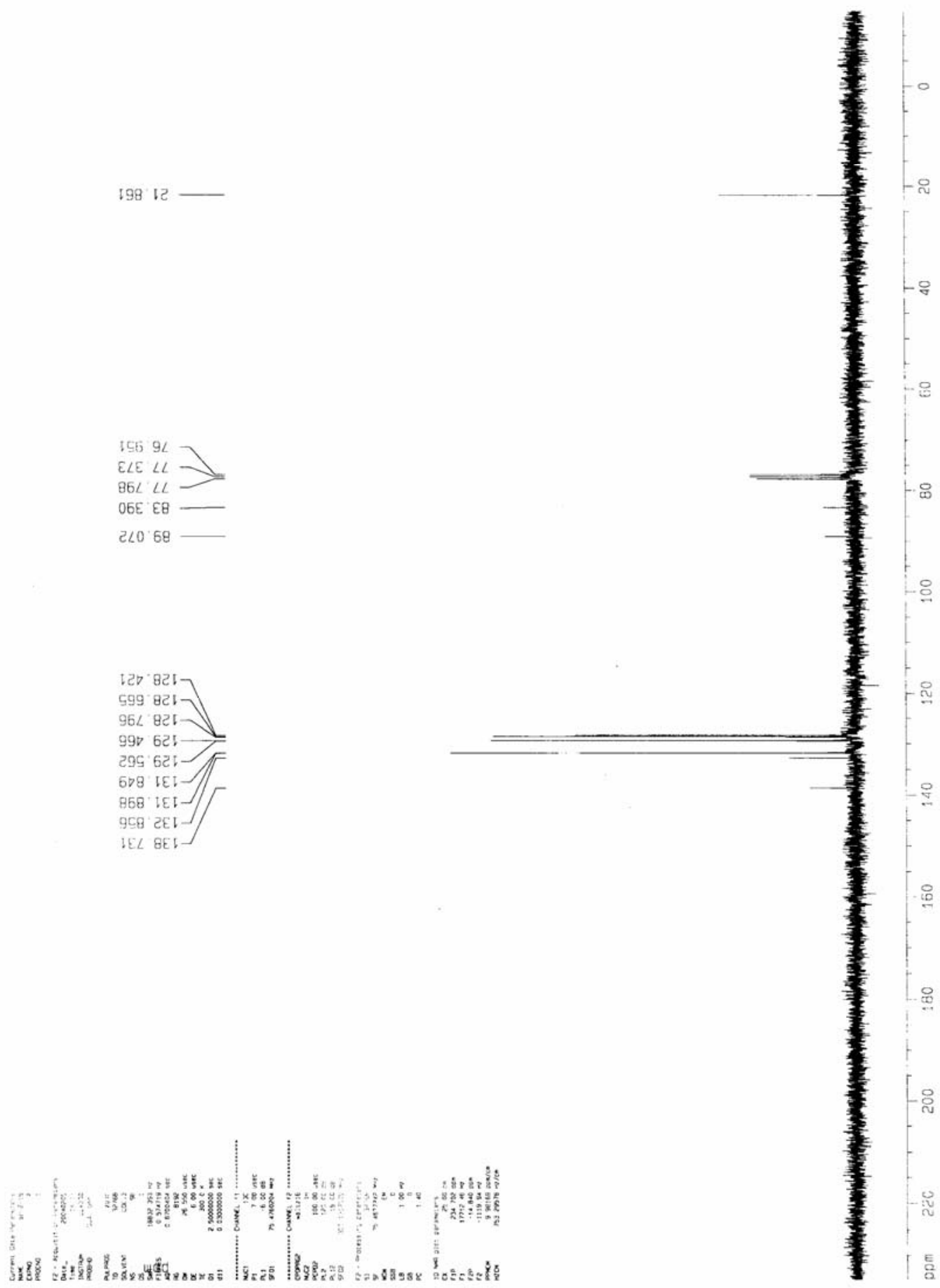


\section{1-(dec-1-ynyl)-4-methylbenzene (5nb)}
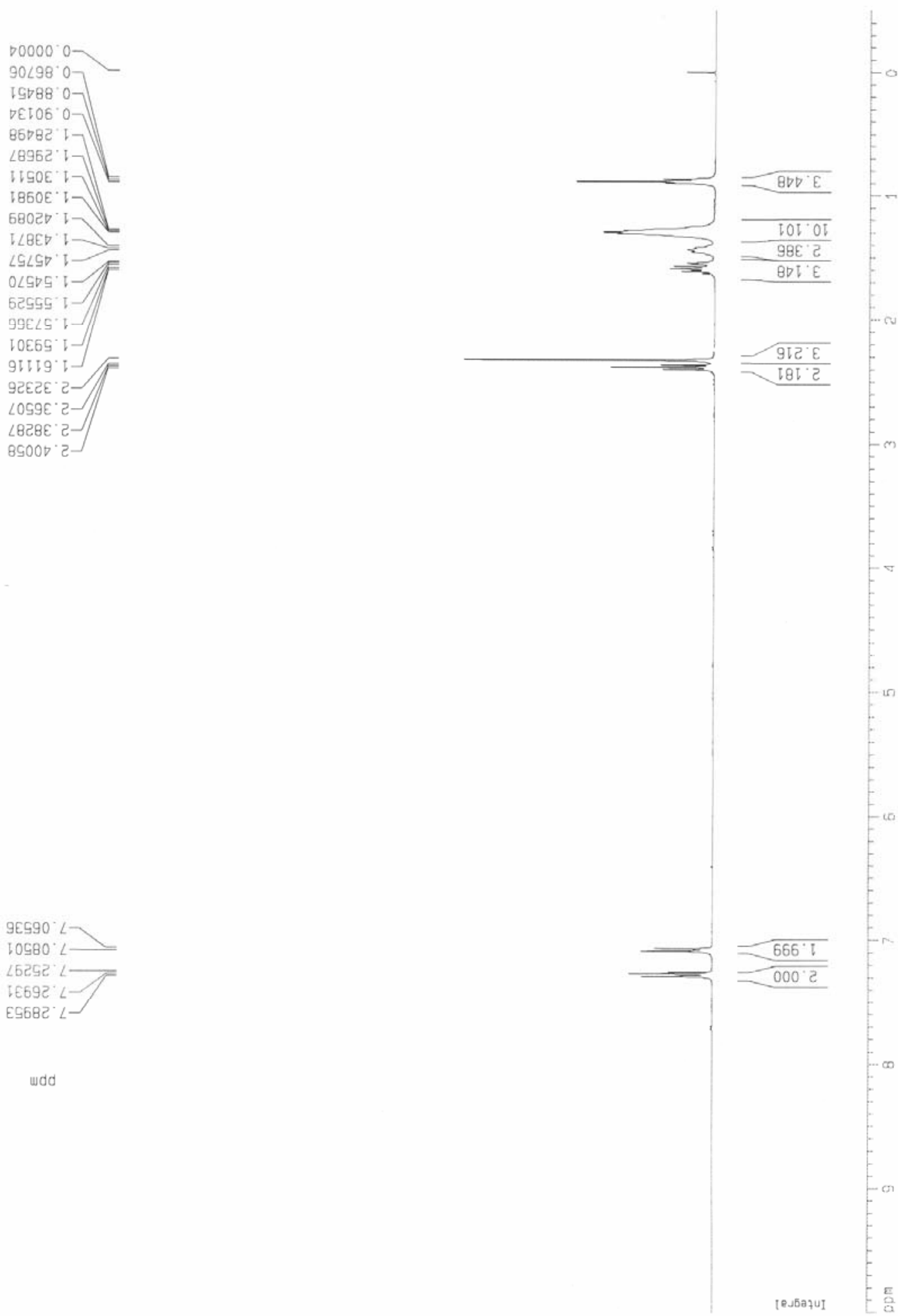
1-(dec-1-ynyl)-4-methylbenzene (5nb)

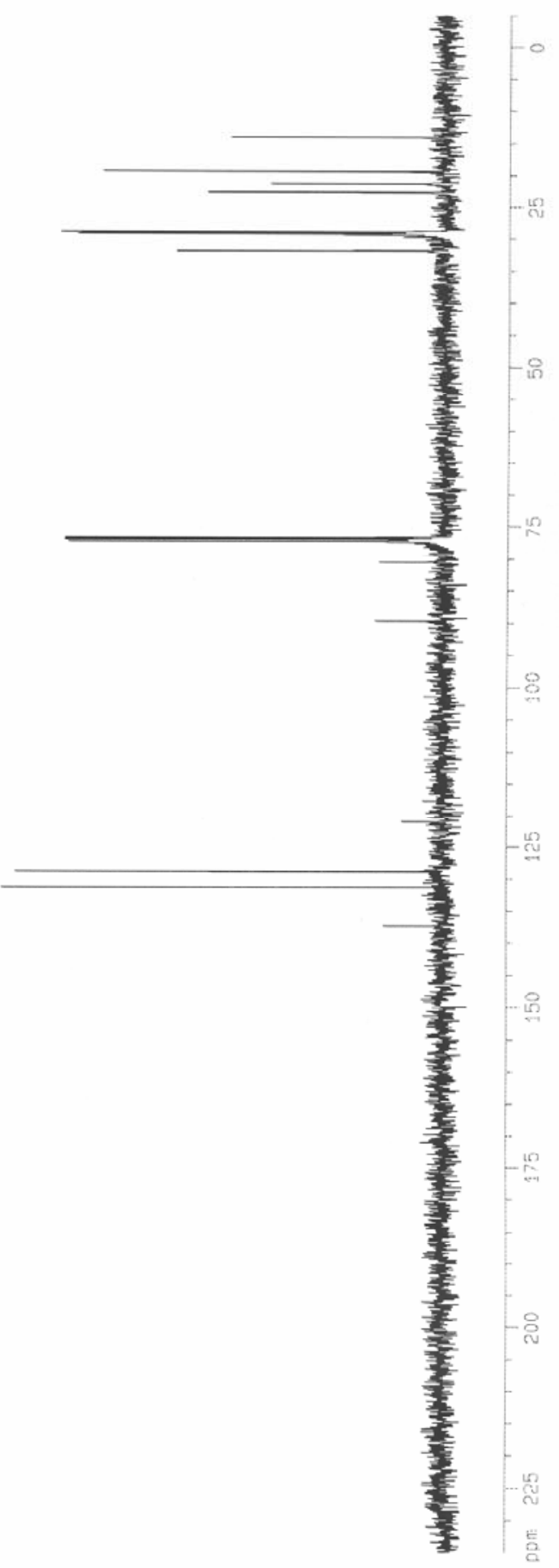


1-(2-o-tolylethynyl)benzene (50a)

$91000 \cdot 0$

L262G '

IG915'2-

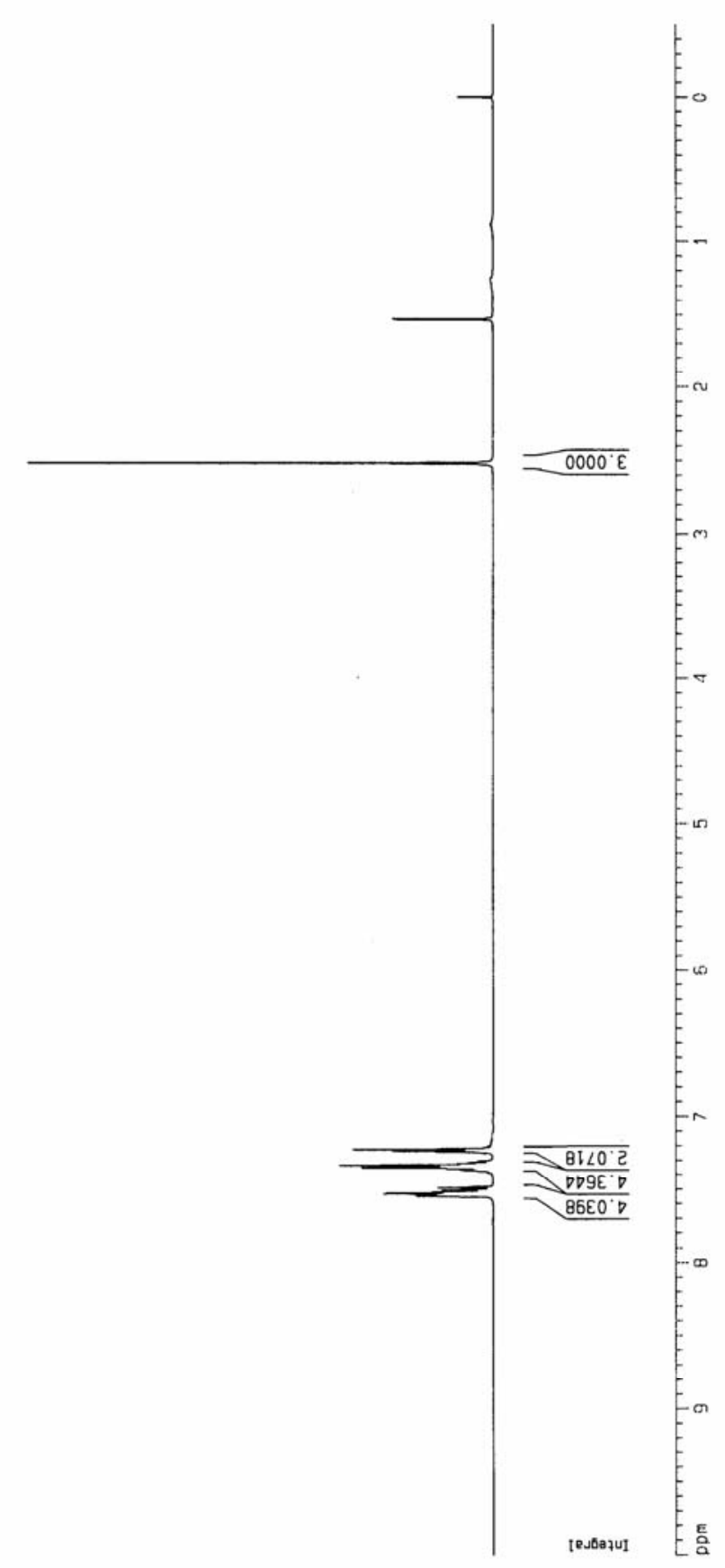

$80022^{\circ} L$

I9ट2) $\angle$

$\angle$ LEEC $\angle-$

EEBCE $L-$

GDOEE $\angle-$

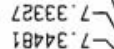

DG9DE $\angle-$

OLGDE $\angle-$

BIEZS ' $L-$

$B E \angle 2 S^{\circ} \angle$

टCDES $L-$

wdd 
1-(2-o-tolylethynyl)benzene (50a)
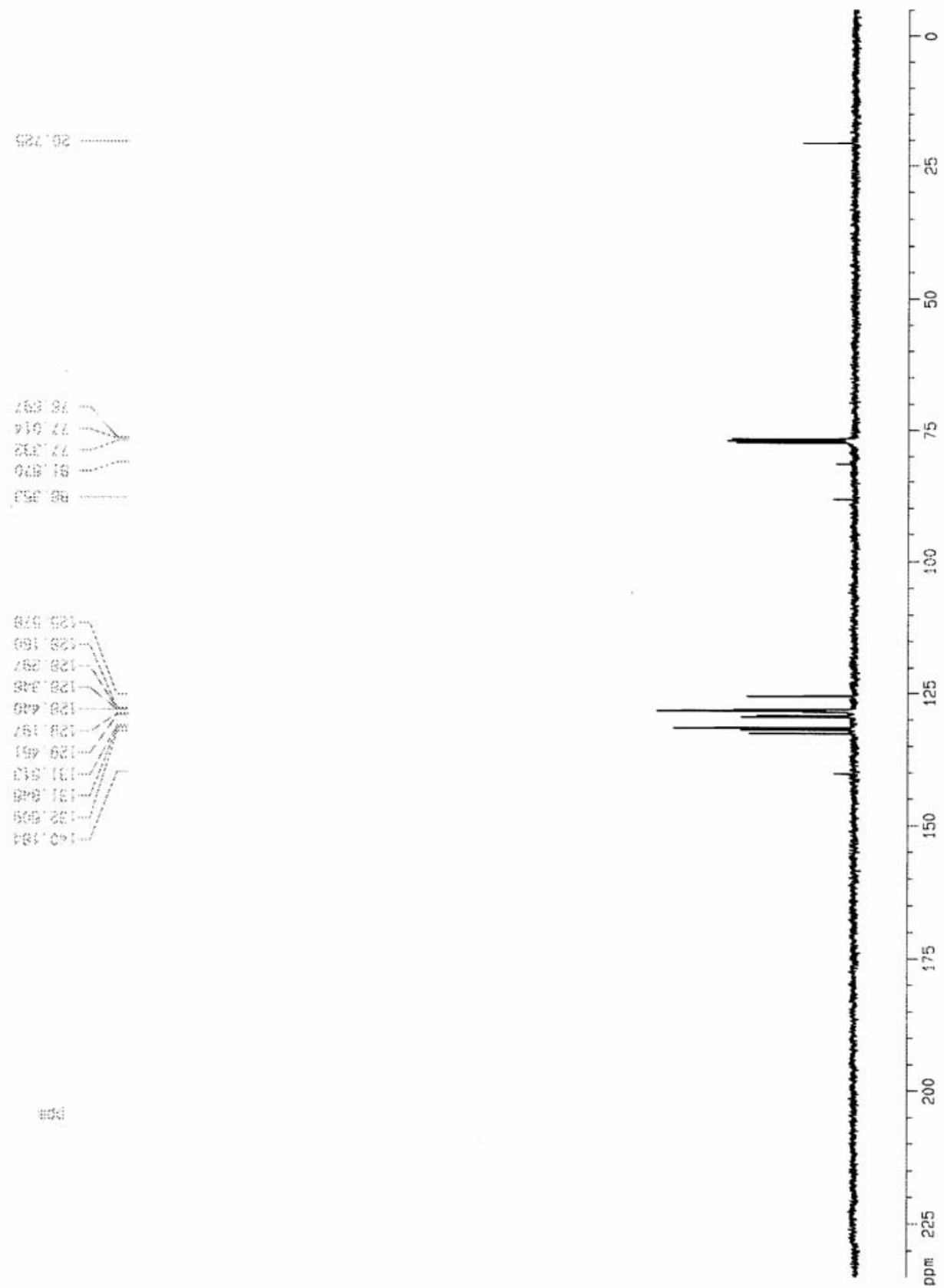
1-(2-(4-Methoxyphenyl)ethynyl)-4-nitrobenzene (5pd)

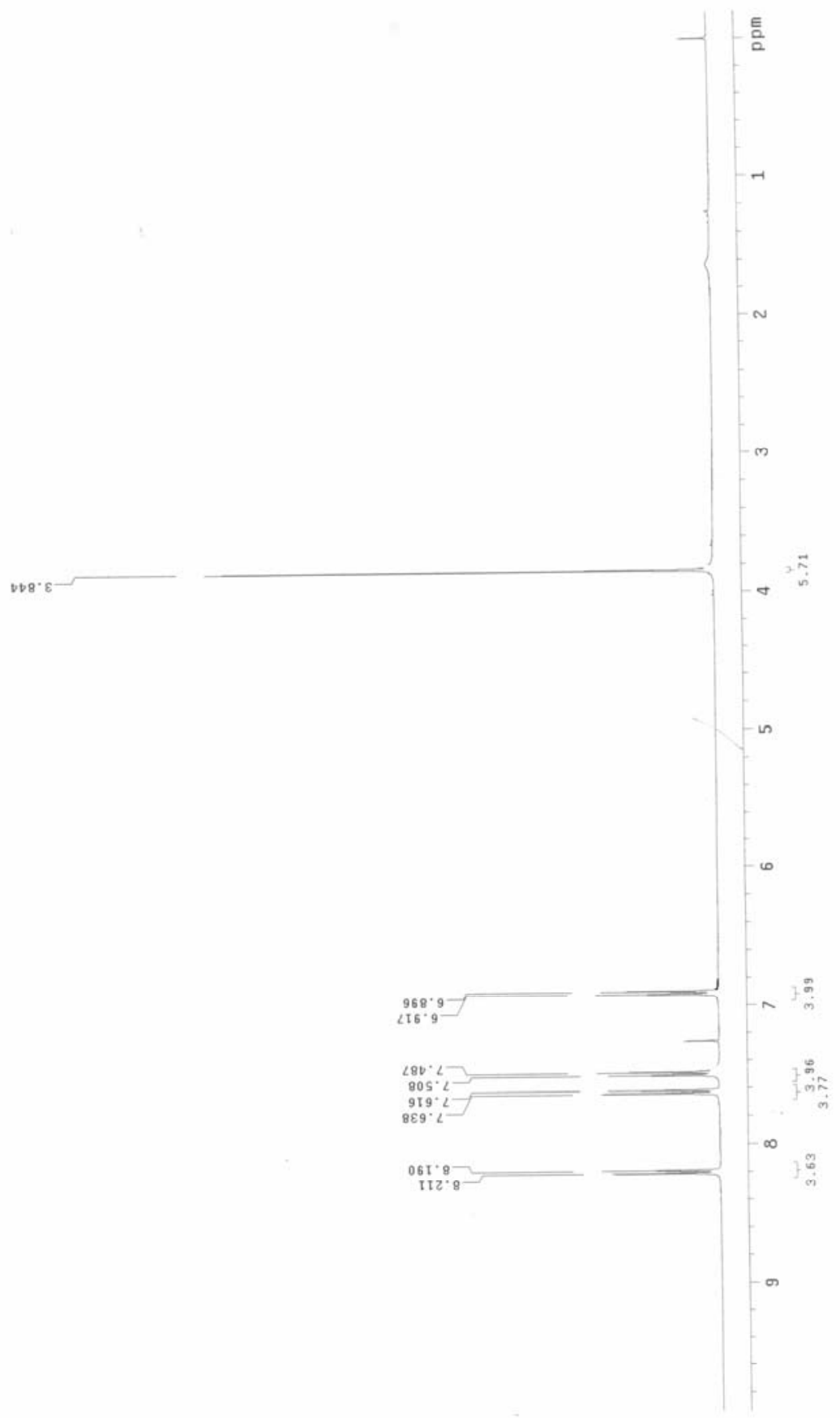


1-(2-(4-Methoxyphenyl)ethynyl)-4-nitrobenzene (5pd)

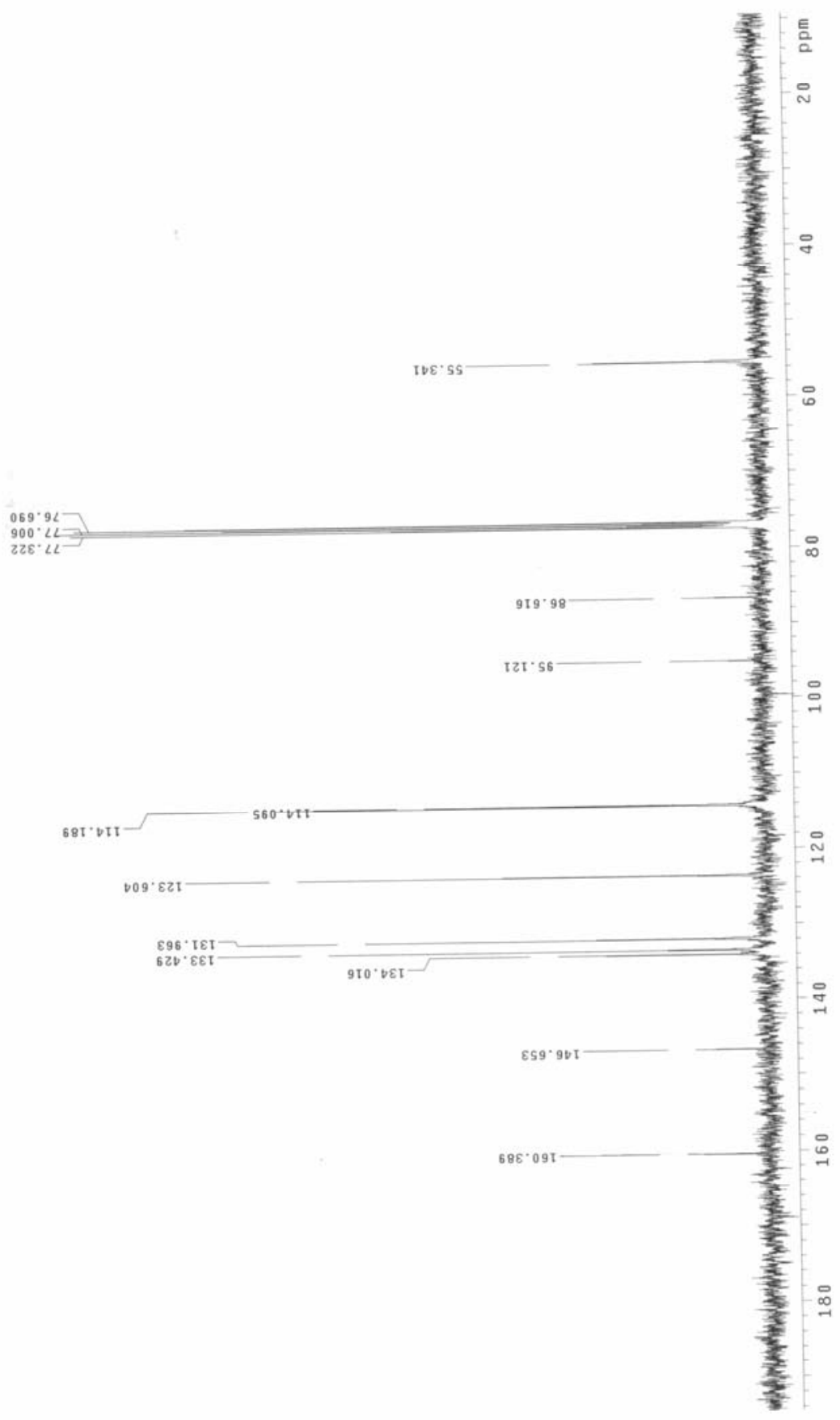


1-fluoro-4-(2-(4-nitrophenyl)ethynyl)benzene (5pe)

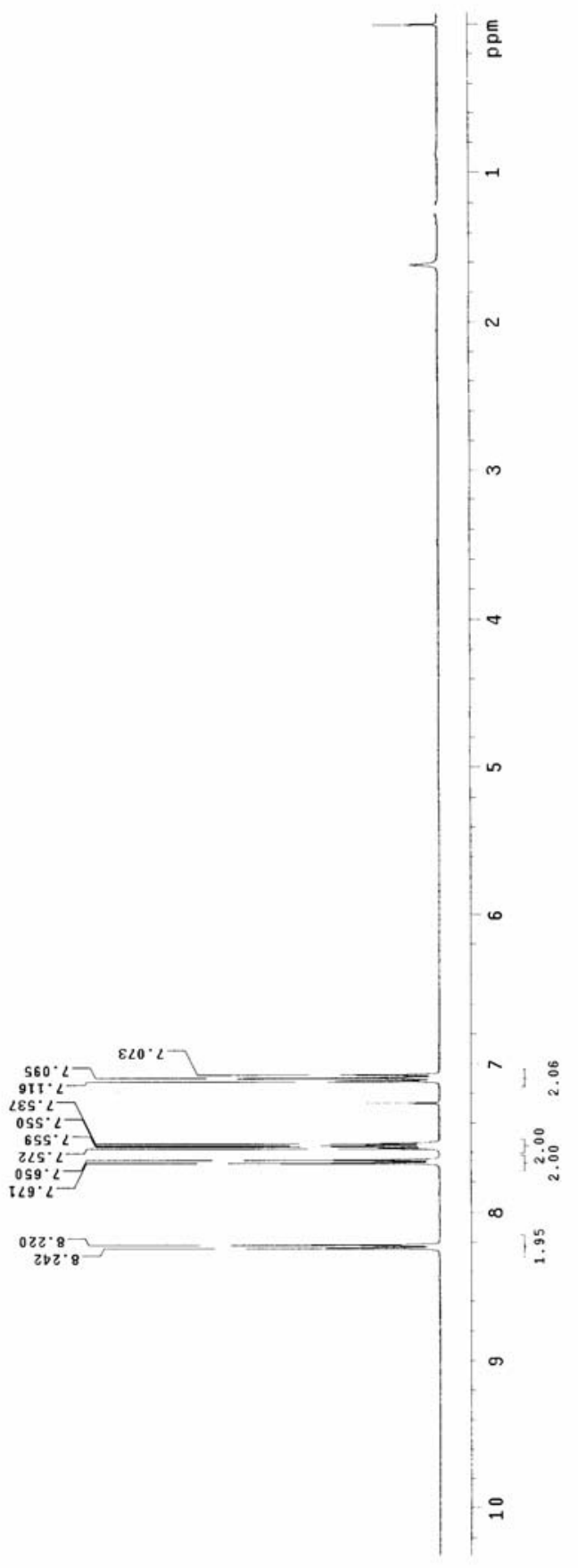


1-fluoro-4-(2-(4-nitrophenyl)ethynyl)benzene (5pe)

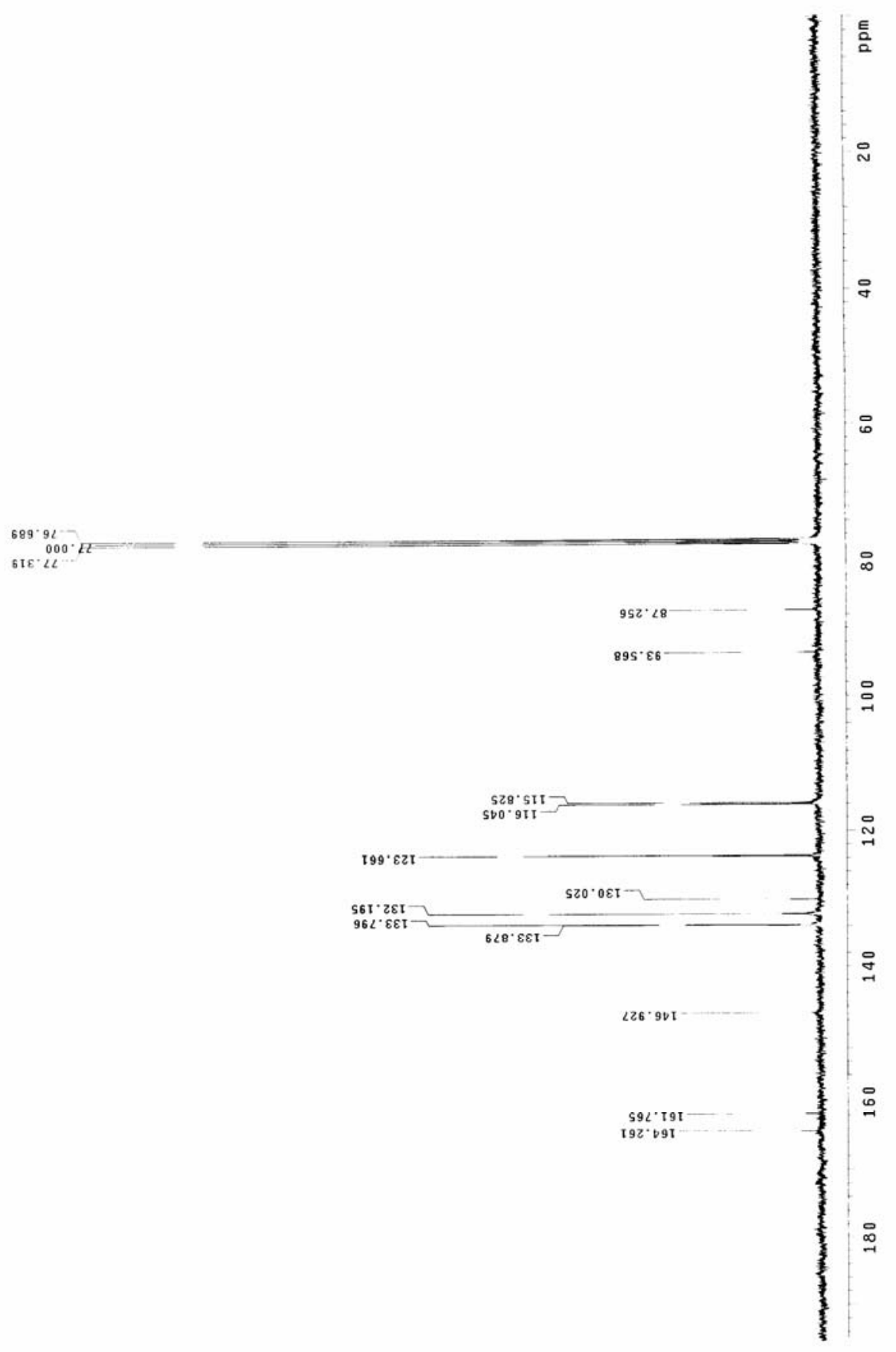


2-Phenylbenzofuran (5va)

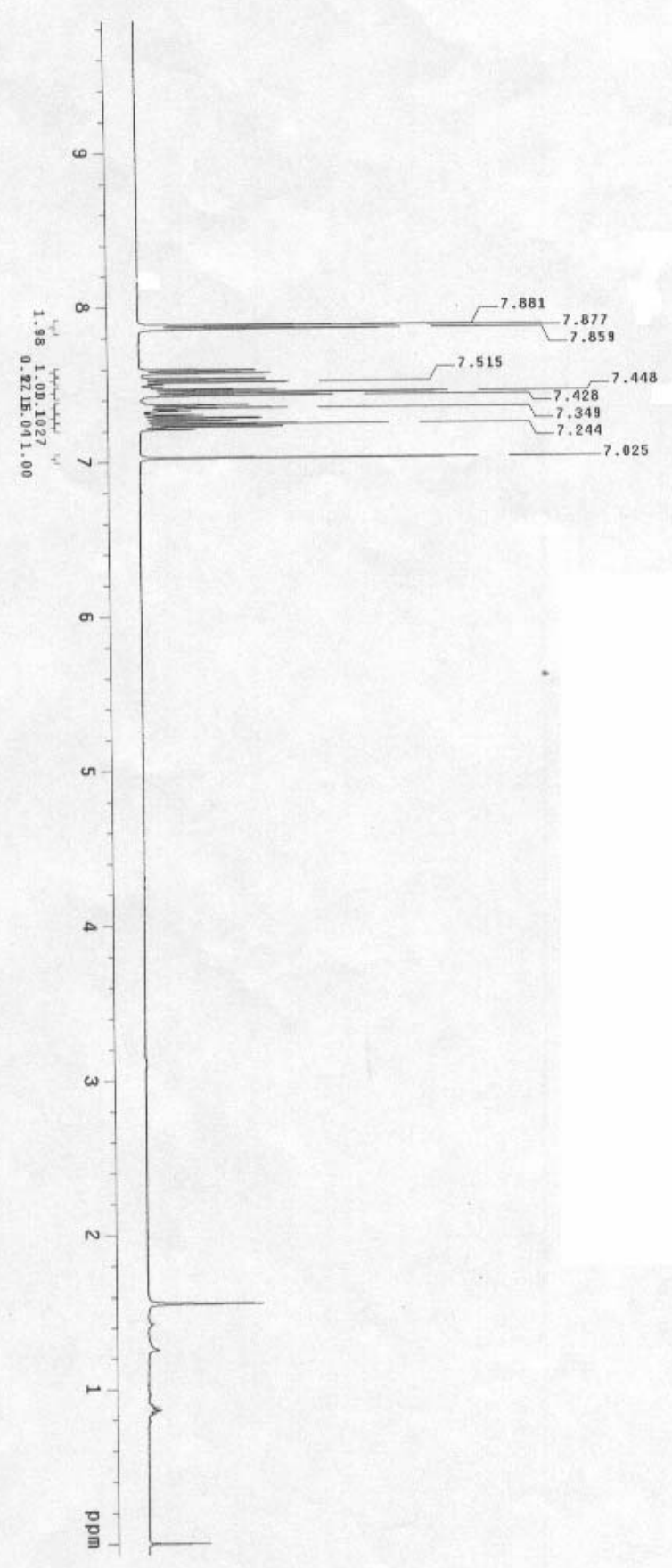


2-Phenylbenzofuran (5va)

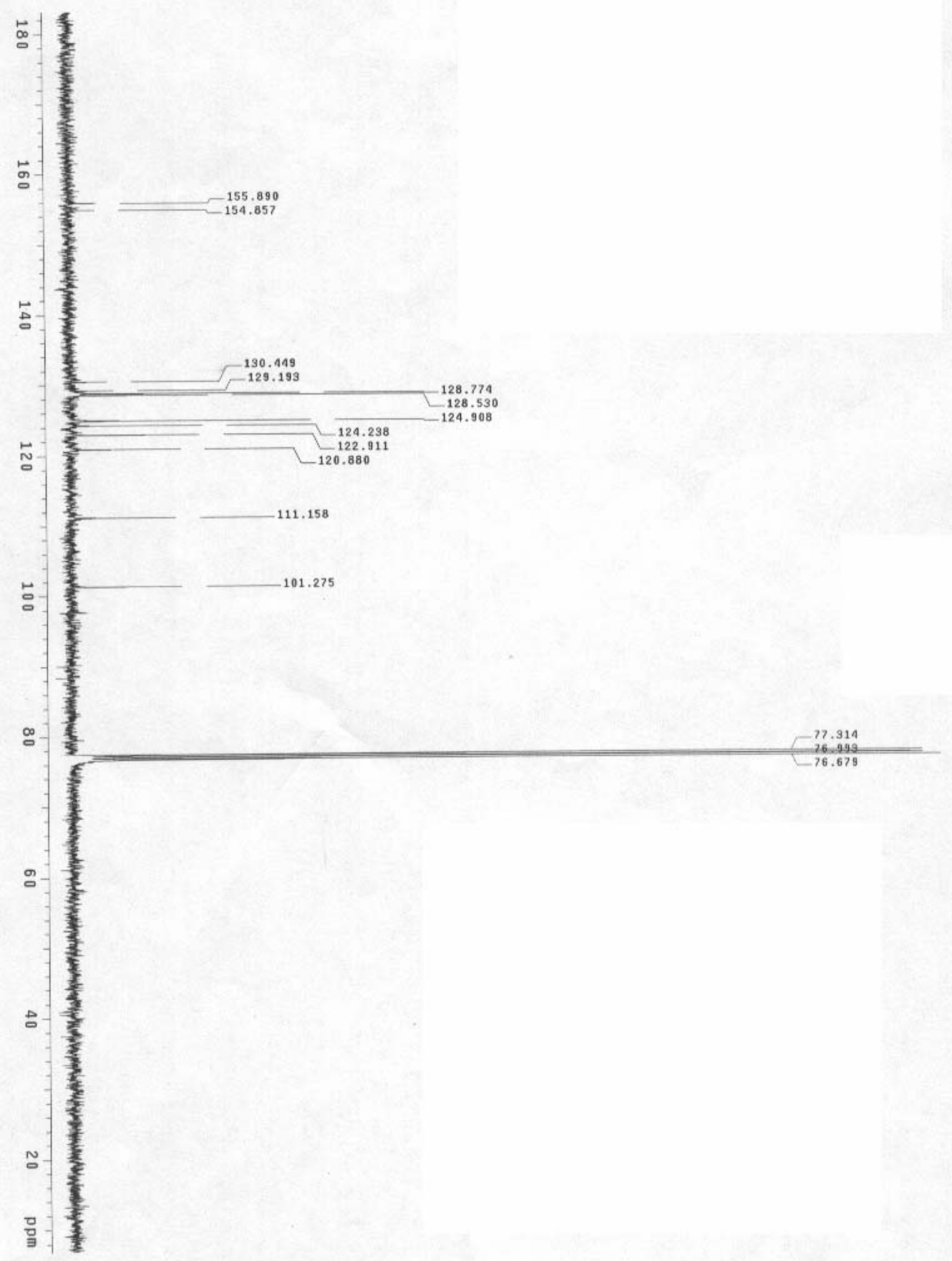


2-Octylbenzofuran (5vb)

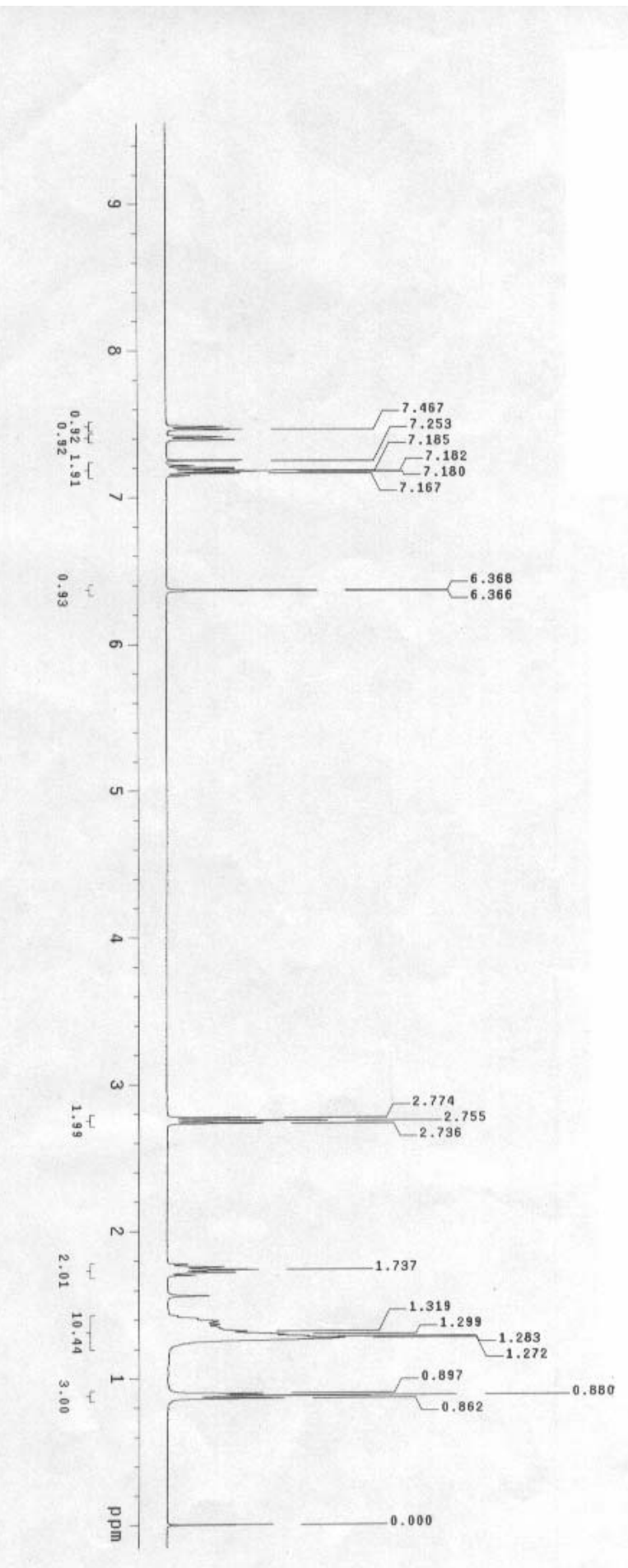


2-Octylbenzofuran (5vb)

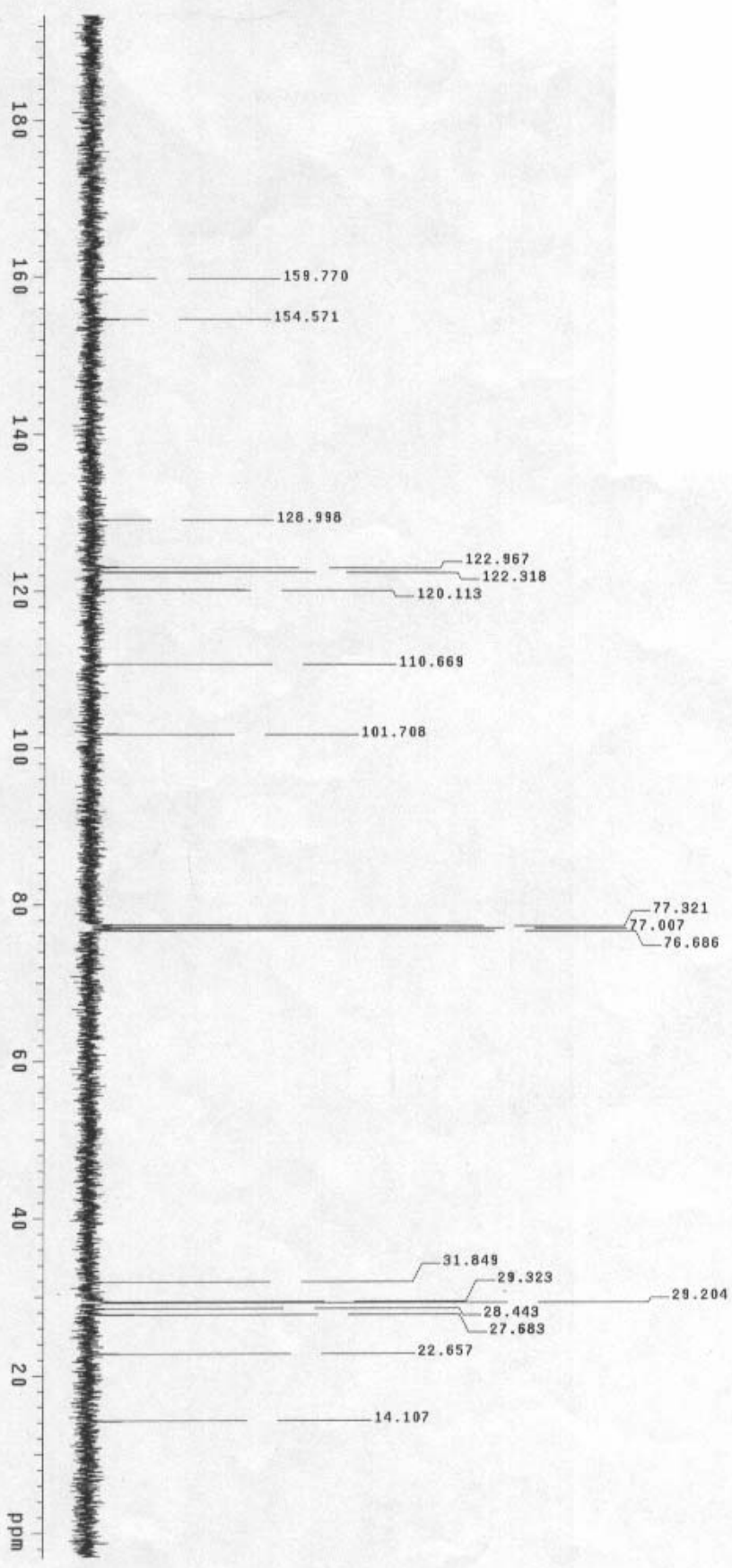


2-Phenylethynylaniline (5wa)

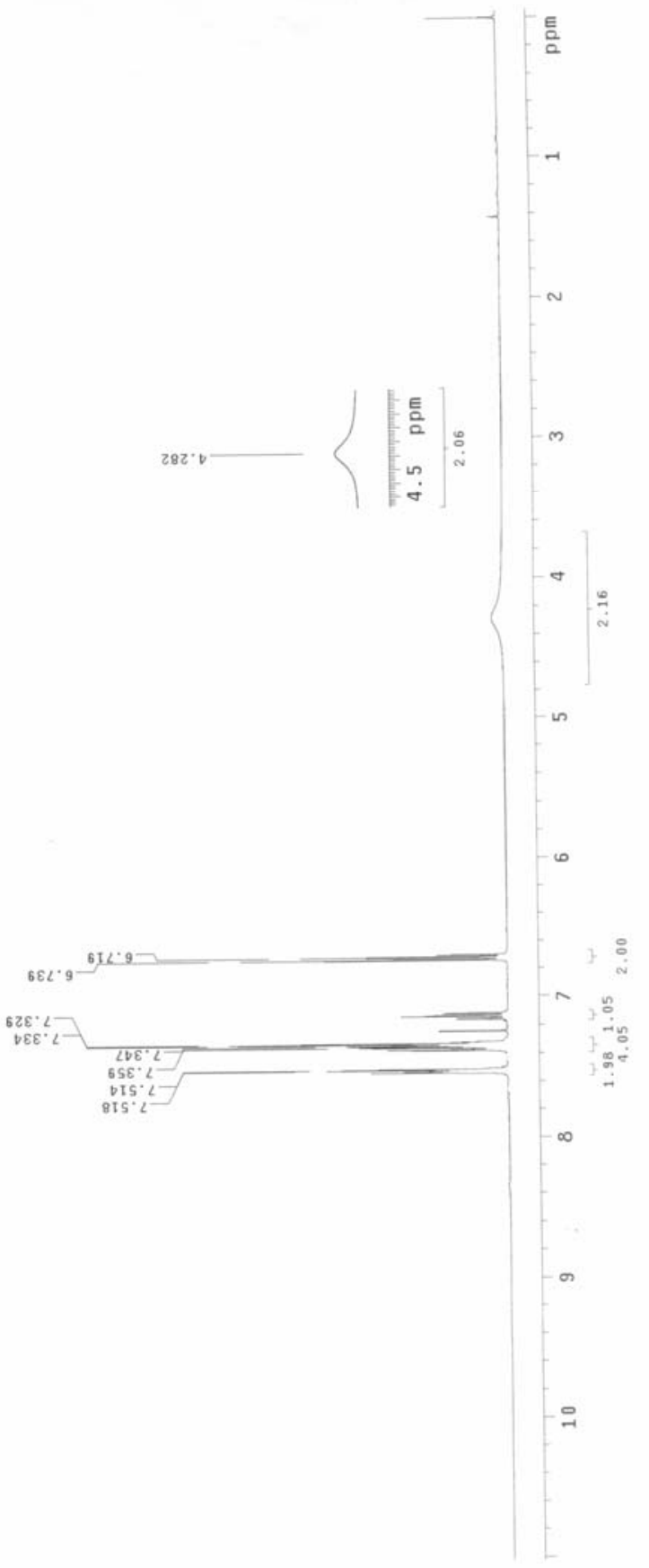


2-Phenylethynylaniline (5wa)

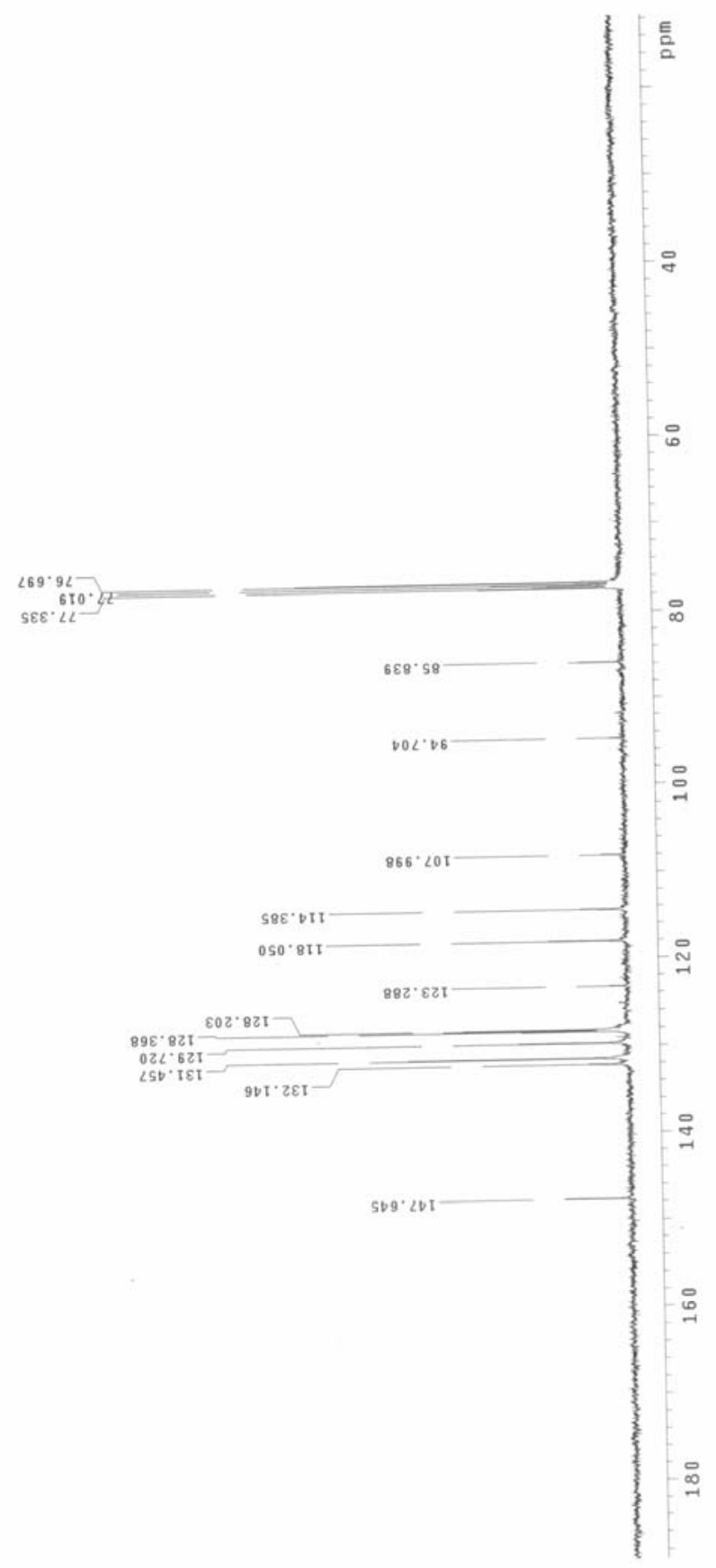

